Jayakody, SA; Gonzalez-Cordero, A; Ali, RR; Pearson, RA; (2015) Cellular strategies for retinal repair by photoreceptor replacement. Prog Retin Eye Res, 4631 - 66. 10.1016/.j.preteyeres.2015.01.003.

\title{
ARTICLE
}

\section{Cellular strategies for retinal repair by photoreceptor replacement}

Sujatha A. Jayakody ${ }^{1}$, Anai Gonzalez-Cordero ${ }^{1}$, Robin R Ali ${ }^{1,2,}$, Rachael A. Pearson ${ }^{1 \$}$

1 Gene and Cell Therapy Group, Department of Genetics, UCL Institute of Ophthalmology, 11-43 Bath St, London EC1V 9EL, UK

2 NIHR Biomedical Research Centre at Moorfields Eye Hospital NHS Foundation Trust and UCL Institute of Ophthalmology, City Road, London EC1V 2PD, UK

Corresponding author: \$ Correspondence should be sent to Dr Rachael A. Pearson, Department of Genetics, University College London Institute of Ophthalmology, 11-43 Bath Street, London, EC1V 9EL, +44 (0) 2076084022 , rachael.pearson@ucl.ac.uk http://www.ucl.ac.uk/ioo/research/ali.htm

Abstract: Loss of photoreceptors due to retinal degeneration is a major cause of blindness in the developed world. While no effective treatment is currently available, cell replacement therapy, using pluripotent stem cell-derived photoreceptor precursor cells, may be a feasible future treatment. Recent reports have demonstrated rescue of visual function following the transplantation of immature photoreceptors and we have seen major advances in our ability to generate transplantation-competent donor cells from stem cell sources. Moreover, we are beginning to realise the possibilities of using endogenous populations of cells from within the retina itself to mediate retinal repair. Here, we present a review of our current understanding of endogenous repair mechanisms together with recent progress in the use of both ocular and pluripotent stem cells for the treatment of photoreceptor loss. We consider how our understanding of retinal development has underpinned many of the recent major advances in translation and moved us closer to the goal of restoring vision by cellular means. 
Keywords: Retina; Stem Cells; Regeneration; Transplantation; Development; Photoreceptor

Acknowledgements: This work was supported by the Medical Research Council UK (mr/j004553/1); BBSRC (BB/1013636/1); ERC (323147-STERD); Fight for Sight (1448/1449); and Macular Vision Research Foundation. A.G.C is supported by Moorfields Eye Charity through a private donation. R.A.P. is a Royal Society University Research Fellow (UF120046) and generously supported by the Alcon Research Institute. R.R.A is partfunded by National Institute for Health Research (NIHR) Biomedical Research Centre for Ophthalmology at Moorfields Eye Hospital and UCL Institute of Ophthalmology (BRC2-007) and the Alcon Research Institute.

The views expressed are those of the author(s) and not necessarily those of the NIHR. Deposited in PMC for release after 12 months. 


\section{Introduction}

As visual animals, our sight is often regarded as our most important sense, with almost $30 \%$ of the sensory input to the brain originating from the retina (Dowling.J.E., 2012; Rodieck, 1998). It makes the loss of this sense a devastating one. Lower vertebrates possess an incredible intrinsic capacity for repair throughout their bodies, including complex neural structures like the retina. The mammalian neural retina, however, like many other regions of the central nervous system (CNS), lacks any significant regenerative potential to replace lost neurons after retinogenesis is complete. Consequently, any damage leading to the death of the light sensing photoreceptor cells or their support cells, either through injury or disease, typically leads to permanent visual impairment. The past decade has seen significant progress in our understanding of the underlying molecular mechanisms for a broad range of retinal diseases. Nonetheless, current clinical treatment options are limited to delaying the onset or decelerating the progress of the condition. To address this, extensive research effort has focused on the development of novel therapeutic strategies. These include, amongst others, attempts to replace damaged cells by transplantation and strategies for the reactivation of endogenous stem cell populations to generate new photoreceptors. The regenerative ability of lower vertebrates has led researchers to investigate the mechanisms underlying these repair pathways to see if the same principles apply in mammals, including humans. Promisingly, a number of studies have demonstrated that some limited regenerative capacity remains in specific regions and cell types in the mammalian eye, including the ciliary epithelium (CE), retinal pigmented epithelium (RPE), iris and Müller glia cells. Endogenous repair is a very attractive strategy and a number of approaches have been employed to attempt to reactivate these cells in vivo (Figure 1a). Thus far, the efficiency of reactivation and the potential of the newly generated cells are currently low and insufficient for the widespread repair of the mature mammalian eye following injury or disease. There remains much scope for improvement in this area, however. Moreover, in vitro culturing of these cells has yielded more hope that they might serve as a source of donor cells for cell replacement therapies (Figure 1b). Effective cell replacement involves the identification of a renewable source of donor cells that have the ability to migrate into and correctly integrate within the recipient retina with high efficiency. Therefore, as well as focusing on ocular stem/progenitor cell niches, we also consider the different types of pluripotent stem cells available for transplantation purposes and our understanding regarding their potential to generate retinal cell types (Figure 1c-d). 


\section{Retinal Degeneration}

Retinal degeneration is caused by the progressive loss of the sensory cells of the retina, the photoreceptors, and accounts for approximately $50 \%$ of all cases of blindness in the developed world (Bunce et al., 2010). Broadly, they fall into three forms: rod-degenerative forms, mixed rod/cone-degenerative forms or debris-associated forms (e.g. mertk defects and light damage). There are many causes of degeneration, primarily hereditary conditions and age related effects, but also diabetic retinopathy or retinopathy of prematurity.

Visual loss from inherited disease includes conditions such as retinitis pigmentosa (RP), choroideremia, Leber Congenital Amaurosis, Stargardt disease and Ushers disease and can arise from mutations in more than 200 different genes [see http://www.sph.uth.tmc.edu/Retnet/; (Hartong et al., 2006)]. As a result, the onset of hereditary disease and the speed of progression can be highly variable, depending upon the specific condition. This contrasts with age-related macular degeneration (AMD), which usually affects older adults (Minassian et al., 2011; Owen et al., 2012; Taylor, 2005). Currently, there are few effective treatments meaning that these conditions not only present a high socio-economic burden for patients and their families, but also for the healthcare system as a whole (Koberlein et al., 2013).

Of the retinal degenerative diseases, RP is perhaps the best characterised (Bird A, 1995), with an incidence of 1 in 3,500-4,000 (Bunker et al., 1984). This group encompasses significant genetic and phenotypic heterogeneity. A wide variety of causes have been attributed; these include disruption of a number of genes that are involved in phototransduction, including the biosynthesis and folding of the photopigment molecule, Rhodopsin, as well as primary failure of the support cells within the retina, particularly the RPE. This heterogeneity means that RP has a highly variable clinical presentation and progression. However, the majority of patients initially experience problems in night vision, since the rod photoreceptors are typically damaged first, and a progressive loss of peripheral vision, leading to tunnel vision. In many cases, this can progress to include the central visual field and blindness (Hartong et al., 2006).

AMD is the leading cause of irreversible blindness in people over the age of 55 (De Jong, 2006) and almost two-thirds of people aged 80 or over show signs of AMD. AMD is a highly complex disease and the mechanism of AMD pathogenesis has not yet been delineated. It is influenced by both genetic and environmental factors. At least 34 different genetic loci are associated with $\mathrm{AMD}$, with the involvement of multiple pathways. A range of single 
nucleotide polymorphisms in genes affecting components of the innate immune system, such as complement factor $\mathrm{H}$, complement factor $\mathrm{B}$ or $\mathrm{C} 3$, has been strongly linked with the disease (Haines et al., 2005; Klein et al., 2005). Modifiable risk factors, such as smoking, diet, cardiovascular disease, high body mass index (BMI), serum cholesterol levels, hypertension and sunlight exposure have also been implicated (Evans, 2001).

AMD is thought to arise as a result of a chronic, low-grade inflammation in the outer neural retina, particularly around the macular region in the central retina. This leads to a degeneration of the RPE and its basement membrane, Bruch's membrane (Jager et al., 2008). Like the conditions described above, it culminates in the loss of both rod and cone photoreceptors, although the cone-rich macular is most severely affected. Vision is lost either from a relatively slow process of photoreceptor death ('dry' or geographic atrophy) or from a more rapid and destructive process of neovascularization through Bruch's membrane and the RPE into the subretinal space and, occasionally, the retina ('wet' or choroidal neovascular AMD). The resulting damage to the macular region leads to a loss of vision in the central visual field. Recent years have seen a major advance in the treatment of neovascular forms of AMD, with the use of anti-vascular epithelial growth factor (VEGF) drugs, which act to reduce or reverse the neovascularisation [reviewed in (Finger et al., 2014)]. However, the dry form of $A M D$ is more common and, like RP, there are few treatment options.

Despite the very diverse aetiologies encompassed within the different retinopathies mentioned above, they share a common outcome: photoreceptor loss. Perhaps surprisingly, much of the underlying structure of the retina, including the second and third order neurons, remains largely intact even after significant photoreceptor cell loss. Santos et al., for example, reported that $80 \%$ of bipolar cells remained in the macular even at very late stages of RP (Santos et al., 1997). It is becoming clear that while these cells survive, they do undergo significant synaptic remodelling, a factor that must be borne in mind for replacement strategies [see section 6 and (Jones et al., 2003; Jones and Marc, 2005)]. Nonetheless, their continued survival and apparent synaptic plasticity presents the opportunity for replacing the lost photoreceptors. This might be by direct replacement with new healthy cells, or with other means of conferring light sensitivity, in the expectation that these remaining inner retinal neurons will be able to process this information and project it to the higher visual areas in the brain. 


\section{Novel Therapeutic Strategies for Retinal Repair}

The eye has several advantages over other CNS structures as a target for therapy development. These include its surgical accessibility, its simple and clearly understood anatomy, extensive understanding of both normal development and disease and its amenability to imaging and functional monitoring. Moreover, the eye is a paired structure and one that is relatively isolated from the rest of the body. This reduces the impact of any systemic adverse effects of treatment. It also allows for a relatively conservative treatment approach, even in the use of very novel treatments, as it is possible to treat one eye and spare the other.

Strategies aimed at halting or delaying the loss of the diseased cells includes neuroprotection and the administration of anti-apoptotic and anti-inflammatory agents. Such strategies do not treat the underlying cause of the degeneration, but aim to slow disease progression and sustain sufficient useful vision throughout the lifetime of an affected individual. As noted above, recent advances in the treatment of wet AMD include the use of anti-angiogenic agents, such as VEGF inhibitors, which are now in routine clinical practice. These inhibitors block the actions of VEGF, which is considered to be largely responsible for the overgrowth of (leaky) blood vessels in the eye in the neovascular forms of AMD.

Neurotrophic factors have been shown to have the potential to delay neuronal apoptosis. Neurotrophic factors are small proteins that have relatively short half-lives and a requirement for repeated administration has limited their clinical application to date. Since these proteins do not ordinarily cross the blood-brain barrier, previous approaches have relied upon intrathecal infusion pumps or similar complex devices to sustain elevated levels of these factors within the CNS. However, sustained delivery through viral vector mediated expression of genes encoding neurotrophic factors may circumvent the potential side effects of repeated administration [for review, see (Buch et al., 2007)]. These include the introduction of growth factors such as pigment epithelium derived factor (PEDF) and ciliary neurotrophic factor (CNTF) (Campochiaro, 2006; Leaver et al., 2006) and reports of sustained delivery of CNTF have been promising in terms of prolonging photoreceptor survival [See (Wen et al., 2012)]. Indeed, CNTF is surprisingly effective in rescuing retinal degeneration due to various causes, including mutations in genes expressed by photoreceptors or the RPE, as well as those induced by strong light, neurotoxins, or antibodies. However, corresponding improvements in visual function remain controversial (Rhee et al., 2013; Schlichtenbrede et al., 2003). Interestingly, stem cells have also been 
used as a means of introducing neuroprotective growth factors into the eye. Wild type and genetically modified human neural progenitor cells survive for prolonged periods, secrete growth factors and rescue models of degeneration, including the Royal College of Surgeons (RCS) rat model of RP (Gamm et al., 2007). The mechanisms by which this protection occurs, however, remains unclear; the initial aim had been to test the cells as a continuous delivery system of glial cell line-derived neurotrophic factor (GDNF), although the wild type cells performed similarly well.

Perhaps the most clinically advanced approach for inherited disorders is gene therapy, the use of viral vectors to reintroduce correct copies of the mutated gene into the diseased cells. This strategy offers the possibility of stopping disease progression, has proven very effective in a large number of animal models of inherited blindness and is now the subject of several clinical trials (Bainbridge et al., 2008; Jacobson et al., 2012; Maguire et al., 2008). A full description is beyond the scope of this review, and interested readers are encouraged to see the special edition of Gene Therapy, 2012 Feb; 19(2), including reviews by (Smith et al., 2012) and an editorial by Ali R.R. (Ali, 2012). Although effective, gene therapy requires a genetic diagnosis and for the cells carrying the mutation to be alive at the time of therapy, often necessitating early intervention. For this reason, and to enable the potential reversal of end stage disease, a variety of complementary approaches are being considered.

An important feature in both animal models of RP and patients with RP or atrophic AMD, as mentioned above, is that despite the death of the overlying photoreceptor cells, much of the inner retinal neuronal architecture, including the ganglion cells, and the remaining visual pathway remain largely intact (Damiani et al., 2012; Mazzoni et al., 2008). Therefore, it may be possible to restore vision by either replacing the missing sensory cells, or by providing visual information to the remaining neurons of the retina, especially the ganglion cells. Strategies for late-stage retinal rescue include cell transplantation, electronic retinal implants, and optogenetic approaches. These latter strategies also do not interfere with the intrinsic progression of disease but attempt to restore photosensitivity by creating new photosensors and coupling them to the remaining retinal circuitry. Cell-based therapy encompasses both the reintroduction of healthy photoreceptors and/or RPE cells into the degenerating retina, as well as cellular regeneration, which involves the potential reactivation of dormant repair mechanisms within the retina itself, stimulating it to generate new photoreceptor cells. Very severe degeneration might be addressed by the transplantation of whole retinal sheets [reviewed in (Seiler and Aramant, 2012)] or the use of electronic devices, which directly stimulate the remaining ganglion cells [reviewed in (Stingl and Zrenner, 2013)]. A more recent and novel idea has been the use of optogenetics to replace 
photoreceptor input by conferring light sensitivity to the remaining inner retinal neurons via the introduction of genetically encoded light sensitive proteins, using viral vectors [see review for further details (Busskamp et al., 2012)].

In this review, we focus our attention on cell-based therapies, first providing a detailed overview of the mechanisms of endogenous retinal repair in vertebrates and the potential to reactivate such mechanisms following injury or the exogenous application of mitogens. Subsequently, we discuss the potential for replacing lost photoreceptors by transplantation together with recent advances in generating transplantation-competent donor cells from a variety of ocular and pluripotent stem cell sources.

\section{Retinal Development}

We begin with a broad description of the regulatory pathways involved in eye and retinal specification, with specific emphasis on photoreceptors. These provide a context within which to evaluate the protocols used for the generation of retina-specific cell types for transplantation purposes, as well as for strategies used to attempt to reactivate endogenous repair mechanisms.

\subsection{Eye Specification}

The eye is a highly specialised extension of the brain. In all vertebrates, eye development involves a series of complex interactions between the neural ectoderm, surface ectoderm, intervening mesoderm and the migrating neural crest cells. Consequently, it provides an accessible insight into neural development and represents an invaluable system for studying the interaction between cells of diverse origins. Shortly after gastrulation, a series of patterning events leads to a single patch of neuroepithelium within the midline of the anterior neural plate, the primordium of the forebrain, being specified to become of eye identity, termed the 'eye field'. Separation of this single eye field laterally results in the formation of the optic primordia, which then evaginate to form the paired optic vesicles. Extension of these optic vesicles allows for contact with the overlying surface ectoderm. This area of contact develops to form the lens placode, and the primordia of other anterior structures such as the cornea and conjunctival epithelium. Simultaneous invagination of the optic vesicles results in the formation of two optic cups (Figure 2a). The inner layer of the optic cup develops into the neural retina and the non-pigmented region of the ciliary body $(C B)$, while the outer layer acquires pigmentation and develops into the RPE and the pigmented region of the $\mathrm{CB}$. The peripheral regions of the optic cup contain the progenitors that form 
the auxiliary structures of the eye, the CB and iris. Notably, while the $C B$, iris and RPE are devoid of neurons and represent tissues both diverse in structure and function in the adult, they share a common origin with the multipotent neuroepithelium [reviewed in (Fuhrmann, 2010)].

While the formation of a functional eye requires the interaction between multiple embryonic tissues, the cellular origins of the neural retina can be exclusively traced to the neural ectoderm (Chow and Lang, 2001). Thus, the formation of the eye is tightly interconnected with the induction and patterning of the neuroepithelium at the onset of gastrulation. Studied by developmental biologists for centuries, one of the earliest stages in the establishment of neural tissue is the decision of ectodermal cells to adopt a neural fate at the expense of an epidermal lineage. At a general level, this is promoted by fibroblast growth factor (FGF) signals, and inhibited by transformation growth factor (TGF- $\beta$ ) and wingless (Wnt) signals. FGF signals are secreted prior to the onset of gastrulation and act to 'prime' the ectodermal cells to later respond to bone morphogenetic protein (BMP) signals, in the presence of low Wnt signals (Chang and Hemmati-Brivanlou, 1998; Hashimoto et al., 2000; Kazanskaya et al., 2000; Linker and Stern, 2004; Mukhopadhyay et al., 2001; Smith and Harland, 1991; Streit et al., 2000; Wolda et al., 1993). Subsequently, to establish and maintain a neural fate, ubiquitously expressed BMP signals must be repressed by antagonists, such as Chordin, Noggin, Follistatin and Cerberus, emanating from the neural organiser (the node in mammals) (Bouwmeester et al., 1996; Hemmati-Brivanlou et al., 1994; Niehrs, 2001). Notably, while FGF signals are required prior to BMP inhibition, this signalling pathway further represses BMP signalling in order to reinforce a neural fate (Rogers et al., 2009). Collectively, it is believed that ectodermal tissue primarily defaults to neural tissue with a forebrain character in the absence of instructive signals (Gamse and Sive, 2000; Houart et al., 1998; Houart et al., 2002; Paek et al., 2009) while posterior neural fates, such as that of the spinal cord, rely upon the presence of posteriorizing factors, including Wnts, FGFs and retinoic acid (RA), in addition to the inhibition of BMPs (Davidson et al., 2000; Durston et al., 1989; Mason, 2007; Nordstrom et al., 2002) (Figure 2b).

Following the establishment of forebrain tissue, rostro-caudal sub-patterning of this region is required to delineate the eye field from the telencephalon and diencephalon, and this process is largely dependent upon the graded expression of Wnts. For example, Zebrafish mutants of axin1, a scaffolding protein required for the down-regulation of Wnt signals, exhibit an expansion of the posterior diencephalic fate, at the expense of the more anterior eye and telencephalon (Heisenberg et al., 2001). Following the establishment of retinal identity, secreted factors emanating from the underlying axial mesoderm are required for the 
separation of the single, centrally positioned eye field into two bilateral symmetrical retinal primordia. While historically believed to be dependent upon the expression of Sonic Hedgehog (Shh) signals from the underlying axial mesoderm, TGF- $\beta$ and FGF signals are also required for this morphological event (Carl and Wittbrodt, 1999; England et al., 2006; Rebagliati et al., 1998; Varga et al., 1999).

These extrinsic signalling molecules act in concert with intrinsic cell determinants to specify neural, and subsequently retinal identity. For example, formation of the neural plate from the definite ectoderm is marked by the upregulated expression of Sox1 (Pevny et al., 1998), and the expression of Gbx2 (Wassarman et al., 1997). Subsequently, neural progenitors contained within the neural tube can be characterised through the expression of several markers including, Sox1, Sox2, Nestin (Lendahl et al., 1990; Pevny et al., 1998; Wood and Episkopou, 1999), Musashi1 (Kaneko et al., 2000) and N-CAM (Ronn et al., 1998; Rutishauser, 1992). Similarly, retinal identity within the anterior neuroectoderm is achieved via the coordinated expression of several intrinsic determinants, termed the eye-field transcription factors (EFTFs) due to their ability to induce ectopic eye-like structures when mis-expressed in the frog embryo. These include Rax, Pax6, Lhx2, Six3, Optx2/Six6, $E T / T b x 3$, and $t / / / N r 2 e 1$ and are expressed within the eye field with overlapping temporal and spatial expression patterns (Figure 3a) [reviewed in (Andreazzoli, 2009) and (Zuber et al., 2003)]. These EFTFs also co-regulate each other. For instance, mis-expression of Pax6 results in the activation of Six3, Lhx2, TIx and Optx2, while overexpression of Six 3 induces Pax6, TIl and Lhx2 (Zuber et al., 2003). Importantly, the molecular network of genes involved in eye formation is conserved between invertebrates and vertebrates, including humans, despite the considerable evolutionary distance and marked differences in eye anatomy and development between the groups (Wawersik and Maas, 2000).

\subsection{Retinogenesis}

Subsequent to eye field formation, the regionalisation of the optic vesicle and, later, the optic cup is reliant upon both extrinsic and intrinsic factors. From this stage, the cells contained within this neuroepithelial structure are known as retinal progenitor cells (RPCs), giving rise to both the neural retina and RPE. Studies in the developing chick have demonstrated that high levels of FGF signalling, derived from the overlying surface ectoderm, are required for the induction of the neural retina tissue from the neuroepithelium; FGF signalling represses the RPE-specific basic helix-loop-helix transcription factor, Mitf, which is required for the acquisition and preservation of RPE fate (Nguyen and Arnheiter, 2000). Repression of Mitf allows for the expression of the paired-like homeodomain transcription factor Vsx2 (formerly 
Chx10), which marks the onset of neural retinal development within the optic vesicle, and as such, this factor is regarded as the earliest retinal specific marker (Figure 3a). This factor is expressed within all RPCs, subsequently becoming restricted solely to the post-mitotic bipolar cells and a subset of Müller glia cells within the postnatal retina. Inactivation of this gene in mice demonstrates that it is crucial for the proliferation of RPCs, especially within the peripheral retina (Bone-Larson et al., 2000; Burmeister et al., 1996). It is important to note that following the formation of these two distinct layers of the optic cup a high degree of plasticity still exists. The RPCs within the presumptive neural retina retain the potential to generate RPE cells (Horsford et al., 2005; Rowan et al., 2004). Additionally, the RPE monolayer is able to differentiate into retinal neurons (Opas et al., 2001; Reh and Pittack, 1995; Stroeva and Mitashov, 1983).

Retinogenesis is highly conserved within vertebrates. Invariably, the first cell type to emerge in all vertebrates is the ganglion cell, followed in partially overlapping phases by horizontal cells, cone photoreceptors and amacrine cells, while the rod photoreceptors, bipolar interneurons and Müller glia cells appear last (Marquardt, 2003; Marquardt and Gruss, 2002). Based upon seminal cell lineage studies, RPCs contained within the developing optic cup have been shown to be inherently multipotent, with a single progenitor cell competent to give rise to all retinal neuron and glia cell types (Holt et al., 1988; Turner et al., 1990; Turner and Cepko, 1987). These findings are encompassed in the competence model of retinal histogenesis, which hypothesises that RPCs undergo a unidirectional change in their competence to generate the different cell types of the mature retina (Cepko et al., 1996).

RPCs are required to undergo several rounds of division to generate a robust pool of progenitors, prior to exiting the cell cycle and differentiating into the retinal cell types. Several growth factors employed during the patterning of the neural tube and eye have been shown to additionally participate in the acceleration and maintenance of this progenitor pool. Much of this work has come from in vitro studies on retinal explants, which have demonstrated that the addition of growth factors, including Shh, FGFs and epithelial growth factor (EGF), to retinal explants in culture is sufficient to increase proliferation (Figure 2b) (Anchan et al., 1991; Jensen and Wallace, 1997; Levine et al., 1997a; Lillien, 1995; Lillien and Cepko, 1992). Supporting these studies, expression analyses have confirmed the presence of these factors from either within the retina or RPE during the period of cell expansion in the developing retina (Chen et al., 2013; Levine et al., 1997a; Lillien, 1995). The responsiveness of the developing retina to such factors has been shown to change over time. For example, it has been shown that earlier progenitor cells preferentially respond to FGF signals, whilst later progenitors are more sensitive to changes in EGF (James et al., 2004; Lillien, 1995). In 
line with the competency model mentioned above, it is believed that gradual changes in intrinsic and extrinsic determinants shift the competence of RPCs to generate all retinal subtypes. Important cell determinants include the basic helix-loop-helix (bHLH)- and homeobox-type transcription factors, which co-ordinate to contribute to the intrinsic properties of retinal precursors, further influencing the determination and differentiation of multiple cell types. While a large number of intrinsic factors have been identified, the full repertoire remains to be established (Cepko, 2014).

\subsubsection{From RPCs Towards a Photoreceptor Cell Fate}

Photoreceptors are born over a long temporal period, which in the mouse spans from embryonic day (E) 12 to postnatal day (P) 10 (Carter-Dawson and LaVail, 1979b; Morrow et al., 1998; Rapaport et al., 2004; Young, 1985). Following the last mitotic division of a RPC committed to generate a photoreceptor cell, these precursors can take up to two weeks after birth to mature and form outer segments. Cone photoreceptors are born first, as early as E11 in the mouse, and their genesis is complete by birth. The mouse has two types of cone opsin, conferring dichromatic colour vision: S opsin (blue-sensitive opsin), which starts to be expressed at E18; and $\mathrm{M}$ opsin (green-sensitive opsin), which is expressed later in development at P6. Conversely, rod photoreceptors are generated over a longer period of time compared to cones, starting at embryonic stage E12 and continuing until P10, peaking at P0. Rod photoreceptors contain only one rod-specific visual pigment, Rhodopsin, which starts to be expressed after birth at P2. As photoreceptors mature, the expression levels of opsins increase substantially, outer segments mature and synaptic connections form with horizontal and bipolar interneurons [reviewed in (Swaroop et al., 2010)].

The development of photoreceptor cells can be broadly categorised into several stages, including (1) proliferation of RPCs to generate sufficient numbers of cells required for a functional retina; (2) restriction in the competence of these RPCs to generate all cell types of the retina; (3) cell fate specification and commitment towards a photoreceptor cell fate; (4) maturation of rods and cone photoreceptors, including the expression of photoreceptorspecific genes essential for function of these specialised cells, and the formation of outer segments and synaptic connections (Figure 3).

Once again, the interplay between both intrinsic determinants and extrinsic factors is required for the correct specification and maturation of these specialised cell types. One of the earliest stages in photoreceptor development relies upon signalling through the Notch receptor (Jadhav et al., 2006; Yaron et al., 2006). High concentrations of Notch allow for the 
maintenance of cycling progenitors, through down-regulating the expression of proneural genes, including NeuroD1, Neurogenin2, Math3, Math5 and Ascl1 (Hatakeyama and Kageyama, 2004; Le et al., 2006). Conversely, inhibition of Notch leads to the production of cone photoreceptors during earlier development and rod photoreceptors at later stages (Yaron et al., 2006). In addition, other signalling molecules including Shh can also influence progenitor commitment through converging downstream Notch targets (Wall et al., 2009). Currently, the molecular mechanisms that underlie the generation of photoreceptor precursors from RPCs, including the down-regulation of Notch signalling, remain to be elucidated.

We have a better understanding of the subsequent events that drive a photoreceptor precursor towards a rod or cone fate. Several transcription regulators central to this process have been identified. These can be regarded as photoreceptor transcription factors, as they are preferentially expressed by either immature post-mitotic photoreceptor precursors, and/or mature photoreceptors (Figure 3b). Over the past years, efforts have been focused on characterising the roles of these photoreceptor transcription factors in an attempt to better understand the development of these specialised neurons, and their role in disease. Our knowledge to date is briefly outlined below. For a more in-depth knowledge of this process, the reader is directly to excellent reviews by (Cepko, 2014; Cepko, 1999; Hennig et al., 2008; Swaroop et al., 2010).

The onset of specification of a precursor cell towards a photoreceptor lineage begins with the expression of two key transcription factors, Otx2 and Crx. Otx2 belongs to the paired-like homeodomain transcription factors and is expressed in RPCs undergoing their final mitotic division and in early precursors committed to a photoreceptor cell fate. Conditional knockout of Otx2, where Otx2 is inactivated under the control of the Crx promoter, leads to the nearcomplete loss of photoreceptor cells in mice, converting photoreceptors to amacrine-like cells (Koike et al., 2007; Nishida et al., 2003), while retroviral gene transfer of Otx2 directs RPCs towards a photoreceptor fate (Nishida et al., 2003). Otx2 directly activates the expression of another member of the Otx homeobox gene family, the Cone-Rod homeobox transcription factor, Crx, in order to cement a photoreceptor fate (Nishida et al., 2003). Crx expression is first detectable in early post-mitotic rod and cone photoreceptor precursors and it is essential for the differentiation, maturation and maintenance of photoreceptors; loss of Crx in mice does not prevent the production of photoreceptor cells, however maturation of these cells fails to occur, resulting in the lack of outer segment, and eventually, retinal degeneration (Furukawa et al., 1999). While these two transcription factors are sufficient to induce a photoreceptor fate, it is likely that other transcription factors, including the PR- 
domain zinc finger protein 1 (also known as BLIMP1), are required to stabilise the commitment of Otx2-expressing cells towards this lineage (Brzezinski et al., 2010; Katoh et al., 2010).

Following commitment to a photoreceptor cell fate, additional transcription factors are required to promote the generation of either rod or cone photoreceptors. Of key importance in this fate is the transcription, $\mathrm{Nrl}$. Nrl is a basic motif leucine zipper transcription factor that is preferentially expressed in rod photoreceptor precursor cells shortly after terminal mitosis, and as such, it is regarded as one of the earliest rod-specific photoreceptor markers (Akimoto et al., 2006). Loss of $\mathrm{Nrl}$ results in the loss of rod photoreceptors and an excess of cone-like photoreceptors (Mears et al., 2001a). Nrl acts in multiple ways to promote a rod lineage, including acting synergistically with $\mathrm{Crx}$ to activate the Rhodopsin promoter. It also directly activates $\mathrm{Nr} 2 \mathrm{e} 3$, which functions to both promote rod photoreceptor genesis and concomitantly repress cone-specific genes (Oh et al., 2008). The peak expression of this transcription factor coincides with rod photoreceptor differentiation, with a subsequent decline in expression during adult stages, however expression is maintained in mature rod photoreceptors, suggesting that it is required for both rod maintenance as well as differentiation (Chen et al., 2005; Peng et al., 2005).

While the commitment towards a cone photoreceptor is less clear than that of a rod fate, several genes have been implicated in this process. The retinoid-related orphan receptor Ror $\beta$ synergises with $\mathrm{Crx}$ to positively regulate the cone S-opsin gene in vitro (Srinivas et al., 2006a). Surprisingly, in vivo knockdown of this receptor results in the loss of rod photoreceptor cells and an excess of S-opsin cone photoreceptors, similar to that seen in the $\mathrm{Nr}^{-}{ }^{-}$mouse (Mears et al., 2001b). Subsequently, it has been shown that loss of this receptor leads to the absence of $\mathrm{Nrl}$, suggesting that Rorß2 lies upstream of $\mathrm{Nrl}$ (Jia et al., 2009). Additionally, it has been shown that Ror $\beta$ acts synergistically with $\mathrm{Crx}$ to initiate S-opsin transcription in cones (Srinivas et al., 2006b). Despite the increase in S-opsin cones in the Rorb $^{-/}$mouse, these cells are non-functional, and more primitive than those in $\mathrm{Nr}^{-/}$mice. In keeping, Ror $\beta$ has been shown to be required for the formation of photoreceptor outer segments, contributing to the development of the inner retina and synaptic layers of the retina (Jia et al., 2009).

The receptors, Rxry and $\operatorname{Tr} \beta 2$, are also required for cone photoreceptor identity. The Rxry receptor is detectable within differentiating cone photoreceptors as well as ganglion cells. In mice, Rxry receptor knockout results in the loss of the ventral-dorsal distribution gradient of $S$ cones and instead results in S-opsin-expressing cones throughout the retina, suggesting 
that Rxry acts in an inhibitory manner to restrict S-opsin expression (Roberts et al., 2005). Separately, the nuclear receptor $\operatorname{Tr} \beta 2$ responds to increasing levels of circulating thyroid hormone during development to direct the differentiation of cone photoreceptors. Specifically, deletion of $\operatorname{Tr} \beta 2$ causes an increase of S-opsin cones at the expense of the $\mathrm{M}$ opsin subtype ( $\mathrm{Ng}$ et al., 2001a; Swaroop et al., 2010).

In addition to their roles in early eye development, several secreted factors have been shown to play an important role in the specification and differentiation of the different classes of retinal cell types. Shh, FGF2, taurine, activin and RA have been all been reported to promote the maturation of rod photoreceptors; exogenous application of these molecules to cultured retinal explants results in an increase in the Rhodopsin-positive cell population (Davis et al., 2000; Hicks and Courtois, 1988; Hicks and Courtois, 1992; Hyatt and Dowling, 1997; Levine et al., 1997b; Young and Cepko, 2004). While less is known about the extrinsic factors important to cone photoreceptor genesis, inhibition of RA in zebrafish has been shown to promote cone neurogenesis (Hyatt et al., 1996) and, as mentioned above, thyroid hormone signalling plays a central role in cone opsin expression and patterning in the retina (Ng et al., 2001b; Roberts et al., 2006).

\subsection{Human Retinal Development}

In comparison to our knowledge of retinal development in lower vertebrates, our understanding of human eye development is far more limited. Classical studies have described the morphological structures of the human embryonic and fetal eye using light (Hollenberg and Spira, 1973; Man et al., 1964; O'Rahilly, 1975; O'Rahilly et al., 1982; Rhodes, 1979) and electron (Hollenberg and Spira, 1973) microscopy. These studies document the timing and morphological changes involved in the formation of the optic vesicles, optic cups and the process of retinogenesis.

Unlike the rodent retina, which has three types of photoreceptor cells (rods and S- and Mopsin cones), the human retina contains four types of photoreceptor cells; the rod and three types of cones (S, L, and M cones) (Nathans et al., 1986). The expression pattern of human proteins during fetal retinogenesis has been described and similar molecular players involved in the development and specification of photoreceptors in lower vertebrates and mammals are also present in the human eye (Hendrickson et al., 2008). Furthermore, the temporal appearance of these markers appears to be conserved. NRL and NR2E3 positive rod cells are detected as early as fetal week 10.5-11 followed by RECOVERIN and interphotoreceptor retinoid binding protein (IRBP) at 12 fetal weeks, and finally components 
of the phototransduction cascade, including RHODOPSIN, PERIPHERIN and ROD ARRESTIN, at 14 fetal weeks (Hendrickson et al., 2008). Much of our understanding of the role of specific transcription factors in human development has been inferred from clinical phenotypes. Heterozygous mutations of OTX2 cause severe ocular malformations (Ragge et al., 2005), while mutations in $C R X$ and $N R L$ have been implicated in Leber's congenital amaurosis (LCA) (Freund et al., 1998; Ragge et al., 2005; Rivolta et al., 2001), cone-rod dystrophies (Freund et al., 1997), RP (Sohocki et al., 2001) and clumped pigmentary degeneration (Nishiguchi et al., 2004). Human genetic studies have, in turn, informed studies in lower vertebrates and animal models, and together have aided the progress of our understanding of both eye development and regenerative processes. Later in the review we describe the advent of mouse and human differentiation cultures from mouse and human embryonic stem cells (ESCs). These open up a whole area of research into human retinal development in vitro.

\section{Lessons from Lower Vertebrates: Endogenous Retinal Repair Mechanisms}

As noted above, current strategies for addressing retinal degeneration are limited to slowing disease progression. One very attractive strategy to reverse the cellular loss is to encourage the retina to repair itself. In the mammalian retina, retinogenesis is completed during development and no neurons are added once the animal has reached maturity. By contrast, classical studies in lower vertebrates have shown that neurogenesis continues through their lifetime. Moreover, they are capable of repairing their retinae following injury, typically via populations of stem cells located in a variety of structures within the eye. Many repair mechanisms are conserved, not only between development and adult regeneration, but also over the course of evolution. For this reason, the study of retinal regeneration in lower vertebrate animals has provided important insights into the potential for retina regeneration in adult mammalian animals, including humans. Indeed, our increasing understanding of these mechanisms has not only permitted the development of protocols for the stepwise differentiation of ocular and pluripotent stem cells for transplantation purposes (see sections $7 \& 8$ ), but also raises the prospect of reactivating these mechanisms in the otherwise quiescent mammalian retina. Classically, three categories of model animals have dominated the field of regeneration; 1) amphibians and fish, which can naturally regenerate after retinal damage; 2) the postnatal chick, which shows a restricted potential for retinal regeneration compared to fish and amphibians; and 3) rodents, which normally do not regenerate following retinal damage, but represent an important model that can allow for new strategies for mammalian retinal repair to be devised and evaluated. In the next section, regions of 
continued retinogenesis within the normal adult eye will be discussed, as well as activation of cells following injury and stimulation with exogenous growth factors.

During development, growth factors influence a variety of cellular processes, including the proliferation, migration and differentiation of stem- and progenitor- cells (Loughlin and Fallon, 1993). Significantly, following injury, many cells can either re-express growth factor receptors in order to respond to local growth factors, or upregulate the expression and release of various growth factors, which can leads to increases in proliferation, migration and differentiation. Of note, the type of injury can play a part in determining which growth factor pathways are activated and, ultimately, the cellular response initiated. Taken together, studies to date have mainly focused on stimulating endogenous stem cell-like cells by intraocular administration of relevant mitogenic factors, in order to reactivate this population.

\subsection{Retinal Repair by the Vertebrate CMZ and CB}

\subsubsection{The CMZ in Lower Vertebrates}

Birthdating studies in fish and amphibians have demonstrated that new neurons are continually added to the retina in concentric rings. These neurons arise from stem cells located at the peripheral region of the retina, termed the Ciliary Marginal Zone (CMZ) (Johns and Easter, Jr., 1977; Straznicky and Gaze, 1971). In a recapitulation of embryonic retinogenesis, cells situated at the peripheral edge of the retina are younger than their more centrally located counterparts. A population of multipotent retinal stem cells residing in the outermost tip of the CMZ express the EFTFs $r x 1$ (the amphibian homolog of Rax1), six 3 and pax6 (Perron et al., 1998). Of note, while retinogenesis continues throughout life, the regeneration potential of the $\mathrm{CMZ}$ cells declines with age [Reviewed in (Amato et al., 2004; Harris and Perron, 1998; Locker et al., 2009; Locker et al., 2010; Moshiri et al., 2004; Perron and Harris, 2000)].

While acting mainly to facilitate the increasing body growth, retinogenesis also allows for cellular repair after injury. The plasticity of the stem cells within the $C M Z$ has been demonstrated following the ablation of particular cell types. For instance, selective destruction of dopaminergic amacrine cells by neurotoxin administration into the eyes of Rana results in the production of this specific cell type by stem cells within the CMZ (Reh and Tully, 1986). Similarly, kainic acid-induced loss of glutaminergic cells stimulates the production of new glutaminergic cells (Reh, 1987). It should be noted however, that despite the common neuroepithelial origin of the neural retina and RPE, these adult retinal stem 
cells are restricted in fate solely to produce retinal cell types, and do not contribute to RPE. Instead, both tissues have been shown to harbour independent tissue-specific stem cells to accommodate any changes through their post-embryonic life (Centanin et al., 2011). With regards to retinal injury, these $\mathrm{CMZ}$ cells are also responsive to the exogenous application of growth factors. Within the fish eye, growth hormone and insulin growth factor-1 (IGF-1) have been shown to regulate neurogenesis, increasing the mitotic index of CMZ stem cells/progenitors (Otteson et al., 2002).

More recently, research groups have focused their attention on attempting to characterise the $\mathrm{CMZ}$ of lower vertebrates, including the identification of candidate genes and signalling pathways involved in the maintenance of this niche (El et al., 2012; Parain et al., 2012). For instance, Parain and colleagues recently conducted a large scale screening to gain insights into the molecular signature of retinal stem cells within the $\mathrm{CMZ}$ of post-embryonic Xenopus tadpoles, revealing 18 novel genes not previously associated with the CMZ (Parain et al., 2012). As might be anticipated, many of the key players involved in embryonic retinal development are also important in the maintenance of the $\mathrm{CMZ}$ in the adult. Denayer and colleagues were able to demonstrate that the canonical Wnt signalling pathway is active within the post-embryonic Xenopus tadpole CMZ, and is required for the proliferation of these cells in vivo without affecting the differentiated cells of the retina (Denayer et al., 2008a; Denayer et al., 2008b), a finding that is also supported by data derived from the fish (Borday et al., 2012; Meyers et al., 2012). Similarly, hedgehog (Hh) signalling has also been shown to regulate the activity of the $\mathrm{CMZ}$, by directly antagonising the canonical Wnt pathway (Borday et al., 2012). For more in-depth information on lower vertebrate retinal regeneration, the reader is directed to the following reviews [(Amato et al., 2004; Locker et al., 2009; Locker et al., 2010; Moshiri et al., 2004; Perron and Harris, 2000)].

\subsubsection{The CB and Peripheral Retina in Mammals}

In 2000, Fischer and Reh reported a proliferative zone at the peripheral edge of the retina in chickens and quails, potentially analogous to the lower vertebrate CMZ (Fischer and Reh, 2000; Kubota et al., 2002). This proliferative region persists for up to three months posthatching. Following the identification of this CMZ-like region in the chick, it was hypothesised that similar regions may exist in other warm-blooded vertebrates. In contrast to the mouse where retinal histogenesis is complete by $\mathrm{P} 11$, the eyes of the marsupial opossum undergo a significant amount of maturation post-birth. In a comparative study of these two animals, Kubota and colleagues found that the opossum, but not the mouse, contains a small 
population of CMZ-like cells at the proliferative margin of the retina (Kubota et al., 2002). Intriguingly, subsequent studies have revealed the presence of a few quiescent retinal stem/progenitor cells that persist at the peripheral margin of the murine retina during early postnatal stages; BrdU-positive cells are still detectable in the anterior margin of the retina until P14 (Abdouh and Bernier, 2006; Moshiri and Reh, 2004; Zhao et al., 2005). Likewise, a developmental study comparing the proliferative potential of dissociated cells isolated from different stages of the postnatal rat retina demonstrated that cells isolated from early postnatal retinas (P1-3) can be clonally expanded in vitro, while cells isolated from P8 retinas have a lower proliferation potential and this property is wholly absent by P14 (Engelhardt et al., 2004). In agreement, we, and others, have shown that dissociated adult retinae (from three-four weeks onwards) fail to give rise to neurospheres in vitro (Ahmad et al., 2000; Kokkinopoulos et al., 2008).

Although endogenous proliferation disappears in the adult mammalian retina, studies have demonstrated that cells at the peripheral margin can be coaxed into maintaining their proliferative state either by the ectopic activation of signalling pathways or in response to disease. Intravitreal injections of insulin and FGF2 induced proliferation of these cells in P14 and P21 rats (Zhao et al., 2005). Consistent with the progressive reduction of the number of retinal stem/progenitor cells with age, this growth factor-mediated response is timedependent, as the same growth factors did not induce proliferation in adult rats (P56-59) (Abdouh and Bernier, 2006). Importantly, and of potential clinical value, a residual population of CMZ-like cells has also been reported within the peripheral region of primate eyes. Martinez-Naverrete et al. demonstrated that a population of NESTIN and PCNA-positive cells exist within the young adult macaque (ages five-six years) and mature human (aged between 45-60 years) (Martinez-Navarrete et al., 2008). Potentially describing the same population, Bhatia et al. examined the effect of culturing human cadaveric eyes in the presence of EGF and observed a significant increase in the expression of the proliferation marker Ki67 in the non-laminated, most anterior region of the retina, when compared to retinae cultured without growth factor supplementation (Bhatia et al., 2009).

A number of studies have sought to determine whether stem cell-like cells at the periphery attempt to repair the diseased retina. In $\mathrm{ptc}^{+/}$mutant mice, which have constitutively activated Shh signalling, proliferating cells can be detected within the retinal margin up to three months postnatally (the latest time point analysed). Furthermore, when crossed to a pro23his degenerative background, the numbers of proliferating cells are additionally increased, with the retinal margin of the $\mathrm{ptc}^{+/}$; pro23his mouse having more than a $50 \%$ increase in BrdU-positive cells when compared to $p t c^{+-}$animals. These persistent 
progenitors attempt regeneration through the production of photoreceptor cells, albeit to a limited extent (Moshiri and Reh, 2004). In the RCS rat model, BrdU-positive and Vsx2positive cells are also significantly increased in the peripheral marginal zone during stages equivalent to mid-stage (P30) and later stage (P60) disease progression when compared with age-matched Long Evans control rats. This appears to be related to the upregulation of Shh pathway, as mRNA levels for Shh, Ptc1 and Smo are increased in the P30 RCS rats compared with controls. In contrast to the previous study though, the increased proliferation does not appear to result in the generation of new retinal neurons (Jian et al., 2009). In addition to the cells in the peripheral margins of the neural retina, a number of groups have documented the presence of cells with proliferative potential within the CB itself. This has generated much interest for many reasons. Firstly, despite being both anatomically and functionally distinct from the neural retina, it is located immediately adjacent and in a continuum with the neural retina, in a region potentially accessible via routine surgical approaches. This has led to interest in these cells as a potential source of patient-derived donor cells for transplantation purposes. Secondly, while no neurons are contained within this region of the eye, the $C B$ is also a derivative of the neural ectoderm, indicating that it could be a potential source for endogenous neural regeneration and repair in higher vertebrates. Within the CB lies a bi-layered epithelium termed the CE. The inner layer of this epithelium is transparent, and is continuous with the neural retina and the iris. In contrast, the outer layer is pigmented and lies between the RPE and the root of the iris (Figure 1a) (Davis et al., 2009).

Despite being quiescent in vivo, two independent groups reported that a population of cells within the rodent CE displayed properties of stem cells in vitro, when dissociated and grown in culture [see section 7.1.1; (Ahmad et al., 2000; Tropepe et al., 2000)]. These cells can be also reactivated in vivo in response to intra-ocular injections of FGF2 and insulin (Abdouh and Bernier, 2006; Zhao et al., 2005). However, although these cells co-express Nestin and either Pax6 or Vsx2, implying a commitment towards a retinal lineage, none were capable of differentiating into retinal neurons or migrating into regions of the neural retina (Abdouh and Bernier, 2006). In addition to growth factor-mediated stimulation, a recent study has demonstrated that inactivation of the Rho family of signalling proteins results in increased proliferation of adult CE cells in situ, an effect that can be augmented when combined with exogenous growth factors (Del Debbio et al., 2014). Likewise, treatment with paroxetine, a selective serotonin reuptake inhibitor commonly used for depressive disorders, results in increased proliferation of this population in vivo (Wang et al., 2010). These studies also report co-expression of Pax6, Vsx2 and Nestin. Markers of a more differentiated phenotype were not assessed (Del Debbio et al., 2014; Wang et al., 2010). 
Reports have also suggested that the stem cell-like cells within the CE can be reactivated following injury. Two independent studies have followed the effects of lesion-induced injuries, either optic nerve transection (axotomy) or optic nerve-crush, on the proliferation of these latent CB stem/progenitor cells in vivo. In the first study conducted in adult mice, Nickerson and colleagues analysed the effects of axotomy on CB stem/progenitors, using the expression of Nestin as a marker for early neural stem/progenitor cells (Nickerson et al., 2007). They described two separate populations of Nestin-positive progenitors within the naïve CB. One population corresponded to pericytes, but the second population was found within the CE. In response to axotomy, these CE-located cells began to proliferate and upregulate the expression of the retinal progenitor marker, Vsx2, although they failed to express Mushashi-1 and Pax6, two additional markers of retinal stem/progenitor cell. In support of this study, Wohl et al. also observed an increase in the number of proliferating cells in the $\mathrm{CB}$ of adult mice following both $\mathrm{ON}$ lesion and crush. Under both injury conditions, similar numbers of Nestin-positive/ BrdU-positive cells were observed, suggesting the activation of the same cell population (Nickerson et al., 2007; Wohl et al., 2009). However, as with those generated following growth factor-mediated stimulation, the proliferative cells are restricted to the $\mathrm{CE}$, and they and their progeny do not extend to the adjacent retina, suggesting an inability to migrate (Nickerson et al., 2007). Interestingly, these cells do appear to have the potential to generate new photoreceptor precursors; Nishiguchi et al. reported an increase in Recoverin-positive cells within the CE, when compared with untreated control animals, following $\mathrm{N}$-methyl-N-nitrosourea (MNU) induced neurotoxic injury (Nishiguchi et al., 2008). However, these cells did not mature, instead remaining morphologically reminiscent of $\mathrm{P} 4$ photoreceptor precursors within the developing retina. They were also small in number, suggesting that their ultimate contribution to retinal function might be limited (Nishiguchi et al., 2008).

Collectively, these reports suggest that the mammalian CE and the peripheral retina contain stem/progenitor-like cells that are amenable to extrinsic stimulation and can undergo limited proliferation and differentiation towards a retinal neuronal fate. However, to date, very few reports have found markers of mature retinal neurons within the cells produced by the $C B$ of adult animals. Moreover, the newly generated cells do not appear to migrate away from the confines of the CB. These cells may either lack the intrinsic capability of differentiating further along retinal lineages, or additional inhibitory cues may exist within their microenvironment to inhibit further migration and/or differentiation. Until we have greater understanding of this niche, these cells represent an intriguing but challenging source for widespread retinal repair of the human retina. 


\subsection{Retinal Repair by RPE}

Located between the retina and the choroid, the RPE in the mature eye is a highly pigmented monolayer of epithelial cells that, under normal conditions, are post-mitotic. Similar to the retina, this structure is a derivative of the neural ectoderm (see section 4.1) but neurogenesis is suppressed upon acquisition of the pigmented phenotype (Gallina et al., 2013).

\subsubsection{RPE in Lower Vertebrates}

Retinal regeneration in amphibians occurs predominantly via RPE transdifferentiation. In salamanders and tadpole frogs, acute injury to the retina stimulates the cells of the RPE to de-differentiate and proliferate to become retinal neurogenic precursors. This process of retinal regeneration from RPE cells has been documented to occur at all stages of development, from early embryonic stages to post-metamorphic stages. Notably, as this process of transdifferentiation occurs, the RPE is replenished as the new retina is formed [reviewed in (Barbosa-Sabanero et al., 2012)]. It is thought that the process of retinal regeneration in these animals is largely regulated by inductive signals released by the extracellular matrix (ECM) (Reh et al., 1987). Indeed, exogenous application of FGF2 is sufficient to stimulate the regenerative properties in the Xenopus laevis stage 51-54 tadpoles following a retinectomy that leaves only the RPE intact (Ueda et al., 2012). The adult newt is capable of regenerating the retina without any extrinsic stimulation and does so by transdifferentiation of RPE cells, with a smaller contribution from the CMZ. To date, the molecular mechanisms underlying these processes remain to be determined; however, gene profiling analyses have demonstrated that the classic retinal stem/progenitor cell markers, including pax6, vsx2, mushashi-1 and notch, are involved [reviewed in (Barbosa-Sabanero et al., 2012)]. In addition to these intrinsic factors, pharmacological application of a Notch inhibitor during transdifferentiation results in premature neural differentiation, implicating the Notch pathway in RPC maintenance and inhibition of retinal differentiation (Nakamura and Chiba, 2007). Finally, more recent studies have shown that in addition to Notch and FGF signalling, cell cycle entry is mediated by heparin, which results in the activation of the Wnt, Shh and thrombin pathways (Yoshikawa et al., 2012). 


\subsubsection{RPE in Mammals}

The embryonic RPE in rats has been shown to possess the ability to regenerate retina via transdifferentiation; this capacity is very restricted, however, being solely confined to embryonic stages (Zhao et al., 2001). Interestingly, during development, proliferation in the immature, undifferentiated RPE is higher than in older, pigmented RPE due to the absence of L-dopa, a key cell cycle regulator and an upstream element in melanin synthesis (Ilia and Jeffery, 1999; Kralj-Hans et al., 2006). This indicates that the capacity for transdifferentiation in the mammalian eye is lost following establishment of the RPE pigmented phenotype. Nonetheless, a proliferative cell population within the peripheral region of the mature RPE has been identified in both human and rat RPE tissue, assessed by the presence of Ki67-, PCNA- and BrdU-positive cells (Al-Hussaini et al., 2008). As noted for similar proliferative cells in the CE, the numbers decline with age from around 20-30 cells per retina at two months of age, to just 8-10 by 1 year. In this study, no markers of neuronal differentiation were assessed. Consequently, while these cells represent a proliferative population of cells within the peripheral region of the retina, whether they can contribute to repair remains to be determined.

\subsection{Retinal Repair by the Iris in Lower Vertebrates}

The iris pigmented epithelium (IPE) is composed of two cell monolayers: the posterior and anterior IPE. The posterior IPE cells are heavily pigmented and are derived from the neural ectoderm, similar to the neural retina, RPE and CB. Under normal conditions, this structure is devoid of neural cells and possesses no potential for generating neurons (Arnhold et al., 2004; Freddo, 1996). The IPE cells of the newt are able to regenerate eye tissue, but only that of the lens. When dorsal pigmented iris epithelium is placed into a regenerating limb, this tissue still results in the formation of a lens. Thus, it is believed that the regenerative potential of the dorsal iris tissue is restricted and unipotent [see (Tanaka and Reddien, 2011) and (Tsonis and Del Rio-Tsonis, 2004) for review]. To date, no reports of mammalian IPEderived regeneration in vivo have been documented.

\subsection{Retinal Repair by Müller Glia}

In the normal healthy retina, Müller cells constitute the principal glia type, adopting many of the functions carried out by the astrocytes and oligodendrocytes in the brain. Present in all vertebrate species, Müller cells are radial glia cells that span the entire depth of the retina with their soma contained within the inner nuclear layer (INL) of the retina [Reviewed in 
(Bringmann et al., 2006; Gallina et al., 2013; Lenkowski and Raymond, 2014; Newman and Reichenbach, 1996)]. While retinal neurons are highly susceptible to various forms of injury, Müller glia cells are exceptionally resistant to ischemia, anoxia and hypoglycaemia, a feature attributed to their particular type of energy metabolism (Silver et al., 1997; Stone et al., 1999). Thus, Müller cells can survive most retinal injuries, acting as a key population of cells available during pathogenic situations. This makes them particularly attractive as targets for regenerative strategies. Below, we discuss some of the key findings regarding Müller gliamediated repair in both lower vertebrates and the mammalian retina, but direct interested readers to the excellent and comprehensive review by (Goldman, 2014).

\subsubsection{Müller Glia in the Fish}

In notable contrast to amphibians (see section 5.2.1), retinal regeneration and repair in fish is achieved by Müller glial cell proliferation. Upon activation, these cells proliferate, albeit for a limited number of divisions, whereupon the progeny subsequently migrate from the INL to the outer nuclear layer (ONL) and differentiate into rod photoreceptors. Using a transgenic zebrafish with a glial-specific promoter (glial fibrillary acidic protein (GFAP)) driving green fluorescent protein (GFP) in differentiated Müller glia cells, Bernardos and colleagues demonstrated that, in fish, Müller glia cells express low levels of the retinal progenitor marker pax6, and proliferate at low frequencies within the mature, intact retina. These progenitor cells express the cone/rod specific transcription factor, crx, allowing for the generation of rod photoreceptors post-embryonically (Bernardos et al., 2007). This study supports previous work by Fausett and Goldman, who used isolated fragments from the goldfish $\square-1$ tubulin gene promoter, which specifically drives GFP expression in Müller glia cells, and demonstrated the appearance of GFP-positive neurogenic clusters following stab wound injury (Fausett and Goldman, 2006).

Interestingly, the key transcription factors sufficient for somatic cell re-programming (the socalled Yamanaka factors, see (Takahashi and Yamanaka, 2006)), c-myc, nanog, sox2 and oct4, are upregulated by Müller glia in regenerating zebrafish retina following injury (Ramachandran et al., 2010b). In addition, many of the EFTFs/RPC genes have been detected within Müller glia and their progenitor derivatives, including six3, pax6, rx1 (Rax) and vsx2 [see Figure 3a; (Bernardos et al., 2007; Lenkowski et al., 2013; Nagashima et al., 2013; Qin et al., 2009; Raymond et al., 2006; Thummel et al., 2010)]. In the zebrafish retina, the transcription factor ascl1 is necessary for Müller glia transdifferentiation and retinal regeneration, and is upregulated within the Müller glia within six hours post-injury (Fausett and Goldman, 2006). More recently, it has been shown that ascl1a can directly drive the 
pluripotency factor and RNA-binding protein lin-28, which further contributes to let7 inhibition, a factor that has been shown to directly repress expression of regenerationassociated genes, including asc/1a, lin-28, oct4, pax6b and c-myc (Nelson et al., 2012; Ramachandran et al., 2010a). Transcriptional repression also plays an important role in retinal regeneration; for example, the transcriptional repressor insm1 suppresses the Wnt antagonist dkk, resulting in the activation of the Wnt signalling pathway, which is required for progenitor proliferation (Ramachandran et al., 2012).

Recently, EGF signalling has been shown to stimulate Müller glia de-differentiation via an epidermal growth factor receptor (EGFR)/MAPK-signalling pathway that impacts on regeneration-associated genes like asc1a, c-myc and pax6, resulting in the activation of both the Notch and Wnt signalling pathways. Furthermore, Wnt signalling has been shown to enhance the stimulation of Müller glia cells both with or without injury (Meyers et al., 2012; Ramachandran et al., 2011), and activation of Müller glia cells following injury leads to the upregulation of Notch signalling components, including deltaC, notch1 and notch3 (Raymond et al., 2006; Yurco and Cameron, 2005). Unlike mammals (see section 5.4.2.), Notch signalling in the zebrafish acts to suppress the formation of Müller glia-derived progenitors in the uninjured retina, whereas inhibition of this pathway leads to the expansion of the region of proliferating Müller glia cells (Wan et al., 2012).

\subsubsection{Müller Glia in Mammals}

In 2000, a seminal paper by Dyer and Cepko demonstrated that mammalian Müller glia could also be induced to proliferate in vivo in response to neurotoxic damage (Dyer and Cepko, 2000). Here, the authors showed that Müller glia down-regulate the tumour suppressor gene, $p 27^{K i p 1}$, within the first 24 hours in response to ouabain and domoic acidinduced injury. This and subsequent studies by Ooto et al. showed for the first time that the mammalian retina is capable of some degree of regeneration when exposed to appropriate stimuli (Ooto et al., 2004). Using the N-methyl-D-aspartate (NMDA) neurotoxicity model of retinal ganglion cell ablation, Ooto and colleagues showed that Müller glia cells re-enter the cell cycle 2 days post-injury in rat, similar to what has been previously reported in the chick and fish (Fischer and Reh, 2003; Raymond et al., 2006; Yurco and Cameron, 2005). Moreover, these newly generated cells upregulate the expression of the neural progenitor marker, Nestin, prior to expressing the mature retinal markers, protein kinase $C$ (PKC) and neuron-specific enolase (NSE) as well as Rhodopsin and Recoverin, markers of early bipolar and photoreceptor cell differentiation, respectively (Ooto et al., 2004). Karl et al. (Karl et al., 2008) found that Müller glia re-enter the cell cycle in response to NMDA retinal damage, and 
upregulate Pax6, Notch and DIl1, similar to the responses reported in non-mammalian vertebrates, such as fish and chicks (Hayes et al., 2007; Kassen et al., 2007; Raymond et al., 2006; Yurco and Cameron, 2007). Finally, following MNU-induced photoreceptor degeneration, Müller glia cells initiated proliferation via a Cyclin D1 and Cyclin D3-dependent mechanism (Das et al., 2006a; Wan et al., 2007). A proportion of the resulting Müller gliaderived cells went on to express the photopigment Rhodopsin as well as Synaptophysin, a presynaptic protein that labels only photoreceptor terminals, suggesting the potential for synapse formation (Wan et al., 2008). In each of these neurotoxic studies, Müller glial proliferation has been proposed to be a consequence of cell loss. Indeed, axotomy and laser injury models have similarly lead to re-entry into the cell cycle (Kohno et al., 2006; Panagis et al., 2005; Tackenberg et al., 2009; Xue et al., 2006). Interestingly, however, sub toxic injections of both glutamate and its analogue $\alpha$-aminoadipate also result in augmented Müller glia proliferation and de-differentiation into neurons in vivo and in vitro (Takeda et al., 2008).

An increase in Nestin expression in Müller glia cells following injury is widely accepted as a marker of a move towards a state more similar to that of a neural progenitor. It is worth noting, however, that Xue et al., postulate that Nestin, an intermediate filament, may provide structural support to the degenerative retina and should not be taken purely as evidence of proliferation (Xue et al., 2006). Notably, the absence of a spontaneous injury-induced response from mammalian Müller glia cells implies that some mechanisms may be in place to limit proliferation of these cells in the adult mammalian retina. At least two, and probably many more, molecular mechanisms are employed within the adult retina to maintain Müller glia cells in a mitotically quiescent state. For instance, TGF- $\beta 2$, which is produced by inner retinal neurons, activates the TGF- $\beta$ receptors in Nestin-positive progenitor cells and GLAST-positive Müller glia cells, stimulating the expression of the cyclin dependent inhibitor, p27 ${ }^{\text {Kip1 }}$ (Close et al., 2005). Furthermore, the EGF receptor expression in Müller glia cells gradually declines as the retina matures, reducing their ability to respond to EGF and again inducing cytostasis. Interestingly, studies of light induced photoreceptor degeneration in albino rats have reported the upregulation of the EGF receptor in Müller glia cells, allowing for these cells to be driven into S-phase by intraocular EGF injections (Close et al., 2006).

Supplementation with FGF2, EGF, activin-A, and insulin alone in the NMDA-injured eye fails to increase the proliferation of mammalian Müller glia cells further than that already activated by injury (Ooto et al., 2004). However, co-application of FGF2 and insulin to the neurotoxininjured (NDMA and KA) retina is able to augment the numbers of activated Müller cells in vivo (Das et al., 2006b). Karl et al. monitored the numbers of BrdU-positive cells within the 
course of a week post-injury and documented that an increase in BrdU-positive cells could be seen as early as $4 \mathrm{~h}$ post-EGF supplementation, but that almost half were lost within $24 \mathrm{~h}$ post-EGF addition. Nevertheless, a subset persisted and could be seen within the retina 30 days post-injection (Karl et al., 2008). While it is not exactly clear why this decline occurs, it could represent the failure of the newly generated neurons to make stable synaptic connections, critical for their survival.

Analyses of activated Müller glia cells following injury have demonstrated that these cells upregulate components of both the Wnt and Notch signalling pathways in response to retinal injury (Das et al., 2006a; Del Debbio et al., 2010; Liu et al., 2013). Exogenous application of Wnt2a, along with NMDA, is sufficient to induce a three-fold increase in the number of injuryinduced stem cell-like Müller glia cells as compared to eyes injected with neurotoxins alone, whereas application of the Wnt pathway antagonist, Fzd-CZD leads to a two-fold decrease in this cell population. DAPT, a Notch pathway inhibitor, has a similar effect. Of note, application of both the Wnt and Notch antagonists results in a lower injury-induced stem celllike Müller glia cell population compared to controls, and levels of CyclinA and CyclinD1 transcripts are also decreased following Wnt and Notch attenuation. Together, these results indicate that the proliferation levels observed after Müller glia activation are likely due to the activation of the Wnt and Notch cascade (Das et al., 2006a). Finally, Shh has also been shown to regulate Müller glia proliferation following retinal injury. Intraocular injections of Shh following $\mathrm{MNU}$-induced retinal damage results in an increase in BrdU-positive cells. Conversely, when injected with the Shh inhibitor, cyclopamine, proliferation is decreased in these retinas, in comparison to controls, suggesting that Shh signalling is activated endogenously following retinal injury (Wan et al., 2007), similar to the Wnt and Notch pathways.

These findings demonstrate that the induction of known developmental signalling pathways after injury can stimulate the regeneration of Müller glia cells following retinal injury, beyond the response to injury alone. In addition to stimulating proliferation, these developmental pathways can also influence the cell fate adopted by these newly generated cells. For example, while supplementation of RA did not induce any changes in the numbers or location of BrdU-positive cells within the NDMA-injured rat retina, the authors reported a specific increase in the proportion of BrdU-positive/PKC-positive cells within the central retina, with no other changes to the differentiation of other retinal neuronal cell types (Ooto et al., 2004). This might indicate that supplementation with RA predominantly promotes a bipolar cell fate. However, subsequent work by Karl and colleagues using a reporter mouse line that expresses enhanced GFP under the metabotropic glutamate receptor 6 failed to 
demonstrate an increase in bipolar cell number following RA stimulation (Karl et al., 2008). The use of the transgenic reporter line would be expected to be the more robust measure, compared to immunohistochemistry; however, the authors only administered a single injection of RA whereas Ooto et al. injected RA three times over the course of a week following NDMA damage. Such modifications in the protocols used may account for the differences in the results obtained. Interestingly, while Shh is normally secreted by ganglion cells and is known to directly control their genesis during embryonic retinal development, exogenous administration of Shh following retinal injury does not result in ganglion regeneration in vivo. Instead the Müller glia-derived progeny preferentially differentiate into rod-like cells, as indicated by an increase in Rhodopsin expression in the degenerative retinas following Shh treatment (Wan et al., 2007).

Müller glial cells within the mammalian retina appear to at least attempt to generate cells, including photoreceptors following stimulation via growth factors and/or injury. However, additional studies are required to confirm whether differentiation is completed and whether or not these new cells are integrated into the existing circuitry. To date, only one study has aimed to address this. Using the S334ter rat model, which suffers retinal degeneration due to the premature termination of the Opsin mRNA translation, Del Debbio and colleagues demonstrated that following Müller glia activation in vivo induced by intravitreal injections of Wnt2b (wnt agonist) and Jag1 (Notch agonist) at P10 followed by Shh and DAPT (Notch antagonist) two days later, a rare subset of GS-positive/Opsin-positive (2.1\%) cells can be detected within the ONL of the retina (Del Debbio et al., 2010). The same group have since reported that these cells are capable of driving the optokinetic head tracking response, where light perception is determined upon the head and neck movement in response to a visual stimulus. Although the number of new cells that expressed Opsin was small, the authors found a significant improvement in head tracking behaviour, close to wild type levels, 17 days after Müller glia activation when compared with control untreated animals (Del Debbio et al., 2014).

In addition to extrinsic signalling cues, intrinsic manipulations can also increase the potential of Müller glia-mediated regeneration. In both fish and chick, Ascl1a is required for retinal regeneration; in fish, asc/1a is upregulated within 6 hours of injury in the activated Müller glia cells, and is required for the increased proliferation and de-differentiation of these cells. While the mammalian homolog, Mash1 is not upregulated in the mammalian retina following injury (Karl et al., 2008), overexpression of Mash1 in dissociated mouse Müller glia cultures and retinal explants demonstrate that forced expression of this gene alone is sufficient to drive the upregulation of retinal progenitor markers, as well as down-regulating glial genes 
through direct chromatin remodelling (Pollak et al., 2013). Following these induced changes, Müller glia cells are able to differentiate into a neuronal cell types as soon as six days postinjury, as determined through their neuronal morphology and the upregulation of many retinal-specific markers, and furthermore, demonstrate signs of functionality in response to various neurotransmitter agonists, including NMDA and kainite (Pollak et al., 2013).

While Ascl1 is sufficient to drive the expression of pan-neuronal and early retinal markers, the expression of photoreceptor genes, such as Rhodopsin and S-opsin is rare. In fact, the newly generated neuronal cells from the Ascl1-reprogrammed Müller glia primarily differentiate into bipolar cells (Pollak et al., 2013). Nevertheless, it may be possible to force expression of transcription factors of known importance during cell fate determination to manipulate the cell fate adopted by these Müller glia-derived cells. For instance, ectopic expression of Math3 and NeuroD with Pax6 is sufficient to promote an amacrine cell fate, whereas the combination of $\mathrm{Crx}$ and NeuroD allows for the generation of photoreceptor cell types (Ooto et al., 2004).

Taken together, it seems likely that the Müller glia within the adult mammalian retina have the capability of proliferating and upregulating early neuronal markers in response to injury, similar to the cells contained with the CE. In contrast to the $\mathrm{CE}$, these cells also demonstrate greater potential for differentiation into mature retinal neurons in vivo, as well as migrating within the neural retina to occupy the correct laminar region appropriate for the newly generated cell type. Given that Müller Glia are distributed throughout the lateral extent of the retina, these features offer the potential for widespread centre-periphery repopulation. However, despite the increase in proliferation exhibited by these cells following damage, the number of new cells generated appears to be relatively low. Ooto and colleagues suggest that reactivated Müller Glia are likely to only undergo a single division following injury before exiting the cell cycle (Ooto et al., 2004). It is not yet clear if it is possible to reactivate these cells in the face of sustained retinal degeneration, as occurs in disease, and if so, for how many divisions. Photoreceptor cells outnumber Müller glia cells in the mouse retina by a ratio of approximately 30:1 (Carter-Dawson and LaVail, 1979a). Thus, for transdifferentiation to be effective, every Müller glia cell would be required to undergo multiple rounds of division in order to adequately replenish the lost photoreceptors cells, while maintaining sufficient numbers of Müller glia cells to support the newly formed photoreceptors, as they do in the normal, uncompromised retina. These remain significant challenges that must be addressed. Nonetheless, the mammalian retina appears to harbour a latent regenerative potential that many hope might be sufficiently exploited in a therapeutic context to restore vision. 


\subsection{Summary of Endogenous Repair Mechanisms in Vertebrates}

In summary, while regeneration and repair is mediated by a variety of ocular cell sources within the lower vertebrate retina, widespread reactivation of these evolutionarily restricted mechanisms in mammals appears more limited. It is possible to induce a number of otherwise quiescent cell types to re-enter the cell cycle, at least as far as S-phase. However, our ability to guide these cells to generate differentiated cell types in numbers sufficient to mediate repair is, currently, poor. Probably the most encouraging, to date, is the potential for Müller glial cell reactivation. This cell type routinely generates new photoreceptors following injury and degeneration in the lower vertebrate retina and recent reports have indicated that it can be stimulated to produce differentiated neuronal cell types, albeit only low numbers of photoreceptors, thus far, in the mammalian retina. Moreover, it is arguably better placed anatomically to enable a widespread repopulation of the neural retina compared to those cell types located in peripheral ocular structures.

\section{Photoreceptor Transplantation}

We have made significant progress in defining the mechanisms that modulate endogenous retinal repair. However, we have yet to reach a state where these may be reactivated in the diseased mammalian retina to a level sufficient to restore visual function. For this reason, many have investigated an alternative, that of replacing the dead cells with new photoreceptors from exogenous sources (Figure 4). The transplanted cells would be expected to function in the same way as endogenous photoreceptors and, since they would connect into the existing circuitry, normal retinal processing would be maintained. It is increasingly clear that there are changes within the remaining retinal circuitry that may influence this processing [reviewed in (Jones et al., 2003; Jones and Marc, 2005; Vugler, 2010)], but nonetheless, the extensive evidence from gene therapy (Bainbridge et al., 2008; Maguire et al., 2008) and the use of retinal implant devices (Caspi et al., 2009; Humayun et al., 2009) demonstrates that it is possible to restore visual function in the degenerating retina. This premise has led to significant interest in the potential for photoreceptor replacement.

\subsection{Defining the Appropriate Donor Cell}

Photoreceptor replacement has largely fallen into one of two strategies; replacement via the transplantation of a whole sheet of retina, often from neonatal sources, or by the transplantation of dissociated cell suspensions. A discussion of the former is outside the 
scope of the current review but can be found in comprehensive reviews by Seiler and Aramant (2012), and Ghosh and Ehinger (2000) (Ghosh and Ehinger, 2000; Seiler and Aramant, 2012). Regarding cell suspension transplants, transplanted cells must migrate from the site of transplantation into the recipient retina, differentiate into photoreceptors and form new synaptic connections to inner retinal neurons. In our own studies, we have described this process as a cell becoming 'integrated' within the recipient retina, while defining the surgical process of delivering the donor cells into the eye as 'transplantation'. Although many studies have routinely used the term integration following transplantation of stem cell-derived retinal cell types, a proper analysis of the "integrated" cell morphology is important. On this basis, we have described photoreceptors as integrated when they (1) show appropriate location in the ONL, (2) one of the inner or outer processes are visible, as well as (3) inner or outer segments and/or (4) synaptic bouton-like structures with the host INL (MacLaren et al., 2006; Pearson et al., 2012) (Figure 4d).

A major challenge has been to identify a donor cell source capable of completing this process of integration. The brain and the retina are both derived from a common source, the neuroectoderm. Given that immature neurons and progenitor cells are intrinsically capable of migrating and differentiating during development, numerous early studies into photoreceptor transplantation examined the potential of brain-derived neural stem/progenitor cells transplanted to the neural retina (Klassen et al., 2007; Mellough et al., 2007; Sakaguchi et al., 2004). Variously, cells have been introduced into the subretinal space (Figure 4c) and the intravitreal cavity. The subretinal space has the advantage of being immediately adjacent to the ONL, where the photoreceptors reside, while the intravitreal cavity is less invasive, being readily accessible via normal surgical procedures. While transplanted neural stem/progenitor cells can migrate into the adult retina, the migration is not restricted to the photoreceptor layer (Sakaguchi et al., 2005; Young et al., 2000). Moreover, these non-retinal sources of donor cells frequently fail to differentiate into mature retinal phenotypes as assessed by immunohistochemistry (Young et al., 2000). More recent studies using tissuerestricted reporter genes have further confirmed that cells derived from such sources that are incorporated into the recipient retina fail to exhibit intrinsic features of mature retinal neurons (Sam et al., 2006).

Many groups have subsequently examined the potential of donor cells isolated from embryonic retinas, which have the intrinsic potential to differentiate into retinal neurons. Different groups have tried transplanting these cells following different in vitro expansion and differentiation protocols prior to transplantation; depending upon the culture conditions used, the cells survive and differentiate into glial cells (Yang et al., 2002) and/or cells expressing 
retinal-specific markers, including some specific for photoreceptors (Qiu et al., 2005), following transplantation. However, they typically fail to correctly integrate into the neural retina, remaining instead at the site of transplantation. Greater success has been achieved when transplanting immature retinal cells into immature recipients, suggesting that the maturation state of the recipient may play a role in determining transplantation outcome: Murine RPCs transplanted into the eyes of neonatal Brazilian Opossums, which provide a foetal-like environment, survived and differentiated in vivo, although integrated cells were not found within the ONL, where photoreceptors normally reside (Sakaguchi et al., 2004). Since the same donor cells failed to migrate into the retina of more mature recipients, it was suggested that the age of the host tissue had a key role in determining the fate of transplanted progenitor cells and their ability to subsequently integrate into the circuitry of a non-autologous retina (Sakaguchi et al., 2003; Sakaguchi et al., 2004; Van Hoffelen et al., 2003).

Poor migration and integration of cells into the host retina could arise from either the intrinsic properties of the transplanted donor cells, from host retinal barriers, or more likely, a combination of both. By using postnatal donor cells from a transgenic mouse ubiquitously expressing GFP and taking these cells from the peak of rod photoreceptor genesis and transplanting them into recipients of exactly the same developmental stage, we found that the transplanted cells migrated into the ONL (and no other layer) of the recipient retina and matured into morphologically normal photoreceptors [Figure 4; (MacLaren et al., 2006)]. Moreover, in contrast to progenitor cells (Sakaguchi et al., 2003; Sakaguchi et al., 2004), these same cells could also integrate with similar efficiency into the non-neurogenic, adult retinal environment [(MacLaren et al., 2006), see also (Bartsch et al., 2008) and (Kwan et al., 1999)]. This indicated that transplantation success depended upon the developmental stage of the donor cell, rather than that of the recipient. The reports by both MacLaren, Pearson et al. and Bartsch et al. demonstrated a strong dependence on the age of the donor cell at the time of transplantation, with efficiency being best when donor cells were taken from around $\mathrm{P} 4$, compared to earlier or later developmental stages. The rod-specific transcription factor $\mathrm{Nrl}$ is first expressed shortly after terminal mitosis [see section 4.2.1.; (Akimoto et al., 2006)]. By using the Nrl.GFP transgenic mouse line, in which GFP expression in the retina is restricted to post-mitotic rod photoreceptors, we were able to demonstrate that effective transplantation is only achieved using post-mitotic photoreceptor precursor cells, cells that are specified to differentiate into rod photoreceptors, rather than progenitor cells or photoreceptors at other stages of development (Lakowski et al., 2010; MacLaren et al., 2006; Pearson et al., 2012). Although we found adult photoreceptor cells to be very poor donors, a report by Reh and colleagues indicates that fully mature photoreceptors can also 
integrate within the wild type retina, but exhibited poorer survival (Gust and Reh, 2011). We have since found that it is indeed possible for mature photoreceptors to integrate, but that they do so with markedly lower efficiency, even when accounting for reduced viability (Gonzalez-Cordero et al., 2013; Pearson et al., 2012). Taken together, photoreceptor transplantation is possible, even into the mature recipient retina, but optimal transplantation is achieved when the donor cells are at the correct stage in development at the time of transplantation (Bartsch et al., 2008; MacLaren et al., 2006) (summarised in Table 1).

The ability to replace cone photoreceptors is of significant importance for clinical application. Cone transplantation has, however, proved challenging. We previously reported that it is possible to transplant small numbers of cones using a Crx.GFP reporter line to isolate photoreceptor precursors at different stages of development (Lakowski et al., 2010). Again, these cells were post-mitotic and their developmental stage influenced the efficiency of cone transplantation, with mature cones integrating very poorly (Barber, Pearson, unpublished data). In each of these studies, the numbers of integrated cones was very low. In a recent study, Ader and colleagues used rods that had been genetically engineered to remove $\mathrm{Nrl}$, turning them into cells with cone-like properties (Mears et al., 2001a). Following transplantation, these cells integrated with moderate efficiency and expressed cone markers, despite frequently adopting a more rod-like morphology (Santos-Ferreira et al., 2014).

Cell-cell fusion is a physiological mechanism that controls a number of developmental processes. In transplantation paradigms, it has also been shown to explain formation of stable heterokaryons and synkaryons following the transplantation of hematopoietic stem cells (Terada et al., 2002; Weimann et al., 2003; Ying et al., 2002). Indeed, a recent study in the retina showed that transplanted hematopoietic stem cells can fuse with retinal neurons in the damaged retina and re-programme them to a more immature state (Sanges et al., 2013). Fusion has been considered unlikely to occur in the retina following photoreceptor transplantation since the donor cells used in the majority of the reported studies are postmitotic, and, to the authors' knowledge, there have been no reports of fusion between two post-mitotic neurons. Nonetheless, the issue of cell fusion following photoreceptor transplantation has been addressed in a number of studies. Donor cells transplanted into knockout models of inherited blindness express the protein missing in the recipient endogenous photoreceptors (e.g. Rod $\square$-transducin in the Gnat1 ${ }^{-/}$knockout, Rhodopsin in the $\left.R h o^{-/}\right)$. In MacLaren et al., the authors used a permanent nuclear marker used to label only the donor cells, which demonstrated the presence of GFP-positive cells within the recipient retina that contained a single nucleus bearing the nuclear label. They also transplanted GFP donor cells into recipients ubiquitously expressing cyan fluorescent protein 
(CFP) and found integrated GFP-positive cells with no co-localisation of the CFP fluorescent signal (MacLaren et al., 2006). In an independent study, Bartsch et al. reported the same finding following the transplantation of donor cells electroporated with tdTomato into recipients that ubiquitously expressed eGFP (Bartsch et al., 2008). In a more recent study, Eberle et al., (2011) used donor cells derived from a double-crossed transgenic line in which eGFP is fused to human Rhodopsin protein and DsRed is expressed under the control of the $\square$-actin promoter. Here, integrated cells expressed both DsRed (cell body) and GFP (localised only to the outer segment) in the correct anatomical locations (Eberle et al., 2011). Note that in the studies described, the recipient retinae are not degenerating; however, a recent report by MacLaren and colleagues has also demonstrated the absence of fusion following transplantation into the degenerating retina (Singh et al., 2013a).

\subsection{Function}

Collectively, there is significant evidence showing that transplanted rod-precursor cells derived from the developing retina are able to migrate into the adult retina and differentiate over a period of several weeks to acquire the specialized morphological features of mature photoreceptor cells (Bartsch et al., 2008; Eberle et al., 2011; Eberle et al., 2012; Kwan et al., 1999; MacLaren et al., 2006; Pearson et al., 2012; Warre-Cornish et al., 2014). However, the fundamental question remained as to whether these transplanted photoreceptor cells could actually improve function. A major challenge for the cell suspension approach was, and indeed still is, to generate sufficient numbers of transplantable cells and in parallel to achieve high and consistent levels of integration. By optimizing the transplantation protocols sufficiently to increase integration $\sim 20-30$ fold, we were able to demonstrate rod-mediated vision after photoreceptor transplantation in a mouse model of congenital stationary night blindness, the Gnat $1^{-/}$mouse (Pearson et al., 2012). A time-course study of the period immediately after transplantation revealed that by 6 weeks post-transplantation $\sim 95 \%$ of GFP $^{+v e}$ cells integrated within the recipient retina expressed rod $\square$-transducin, the protein missing in the endogenous photoreceptors of the recipient, in their outer segments (WarreCornish et al., 2014). Suction electrode recordings revealed that the newly integrated donor rod cells are light sensitive and display dim-flash kinetics very similar to wild type rods. Moreover, the dim light-evoked signals generated by transplanted rods are projected to higher visual areas, including V1 of the visual cortex, and are capable of driving at least some forms of visually-guided behaviour (Pearson et al., 2012). These findings support previous studies of single measures of function, including simple light-dark preference and wheel running tests following the transplantation of retinal cell suspensions derived from early postnatal retinae (Klassen et al., 2004; Kwan et al., 1999). Takahashi and colleagues 
recently reported a physiological characterisation of Nrl.GFP donor cells transplanted into the $\mathrm{Crx}^{-/}$recipient, which lacks outer segments and is non-functional, and similarly reported that integrated donor cells formed new outer segments and presented $\mathrm{Ca}^{2+}$ responses and rectifier outward currents similar to wild type photoreceptors (Homma et al., 2013). The electroretinogram (ERG) is recorded at the corneal surface and is an average of the electrical responses of all the cells in the retina in response to a light stimulus. It is one of the most commonly used assessments of visual function. Despite robust integration of transplanted photoreceptors, however, the levels achieved thus far still appear insufficient to drive a robust ERG response in the recipient retinae (Pearson et al., 2012). By using gene replacement in the same model, we found that approximately 120,000 functioning rod photoreceptors were required to generate a reproducible scotopic ERG response (Pearson et al., 2012). Taken together, there is an increasing body of evidence demonstrating the ability to restore visual function in the diseased retina following the transplantation of immature photoreceptors (see also, section 8.2.).

\subsection{Challenges Facing Transplantation}

The work of the last decade or so has demonstrated that photoreceptor transplantation is possible and that the new cells are capable of driving visual function. However, a number of major challenges must be addressed before this strategy can be applied clinically. These include, amongst others, the achievement of high levels of integration and long-term survival of donor cells within the severely degenerate retina and the establishment of protocols for the robust generation of transplantable populations of donor cells from a renewable source. The most successful transplants have, thus far, been achieved either in wild type animals or in models with only slow to moderate degeneration. Donor cells transplanted into the subretinal space must migrate out from the cell mass, through the recipient interphotoreceptor matrix (IPM). They must then cross the outer limiting membrane (OLM), a series of adherens junctions formed between photoreceptors and Müller glia, before migrating through the ONL to an appropriate position (Warre-Cornish et al., 2014). Thus, in addition to the donor cell being at the correct stage in development, their interactions with the recipient retinal environment are also likely to be key in determining transplantation outcome. Accordingly, the diseased retina presents a new set of challenges. A comprehensive review of the challenges presented by the disease retina to photoreceptor transplantation is beyond the scope of this review, but is considered in depth elsewhere (Pearson, 2014). 
Briefly, the cytoarchitecture of the recipient ONL becomes disrupted and the OLM can in some, but not all, cases become compromised (RAP, unpublished data). The latter may be beneficial to transplantation since the OLM may represent a physical barrier to the migration of donor cells into the ONL (Pearson et al., 2010; West et al., 2008). However, another major feature of retinal degeneration is Müller glial cell reactive gliosis; the resulting glial scar can envelop the entire retina at late stages of degeneration (Jones et al., 2003). Not only does this scar form a physical barrier between injured and healthy tissue, it can also act as a reservoir for the accumulation of inhibitory ECM proteins, including chondroitin sulphate proteoglycans (CSPGs), which are known to be inhibitory to axonal regeneration (Theocharis et al., 2010).

We recently reported the first comprehensive study comparing rod transplantation into 6 different models of inherited degeneration (Barber et al., 2013). Importantly, the severity of degeneration was not a determining factor in transplantation outcome and robust donor cell integration was observed, even at late stages of degeneration in some (Barber et al., 2013), as also reported by others (Kwan et al., 1999; Singh et al., 2013b). This is important since, at least initially, such novel therapies would likely be applied to only very advanced cases. However, the aetiology associated with a given disease type was shown to impact significantly on both the number and the morphology of integrated rods.

In particular, we noted an inverse correlation between the extent of gliosis in the recipient retina and the number of integrated cells (Barber et al., 2013). Glial scarring has been proposed to explain the lack of integration of retinal sheets with the host retina, as neurite extension does not occur in regions of reactive gliosis (Zhang et al., 2003). Similarly, dissociated hippocampus-derived neuronal progenitors integrated poorly in the adult retina of dystrophic rats compared with non-dystrophic animals (Young et al., 2000). Confusingly, gliosis has been reported to have beneficial, as well as negative, effects on transplantation strategies; Nishida et al. reported that the upregulation of intermediate filament proteins following retinal damage may actually promote the survival of transplanted neuronal stem cells (Nishida et al., 2000). Gliosis is complex process, however, and assessments made on the basis of intermediate filament protein expression alone may be misleading. CSPGs are upregulated in response to injury and disease throughout the CNS (Fawcett, 2009; Gumy et al., 2010; Inatani et al., 2000) and participate in the inhibition of axon regeneration (Crespo et al., 2007; Friedlander et al., 1994; Gilbert et al., 2005). With respect to transplantation, they are likely to present a repulsive barrier to transplanted donor cells. We have found very different patterns of CSPG expression in different models of degeneration, as assessed with the broad-spectrum CSPG antibody CS-56 (Barber et al., 2013) (Hippert, Graca et al., in 
press). Moreover, levels of CSPGs in the recipient IPM are inversely correlated with the number of integrated donor cells post-transplantation (Barber et al., 2013). Strategies that prevent the formation of inhibitory CSPGs, or break them down, are of significant interest for improving the outcome of cell regeneration and replacement strategies. The bacterial enzyme chondroitinase $A B C$ (ChABC) degrades CSPG side chains and has been reported to promote functional recovery (Bradbury et al., 2007; Crespo et al., 2007) and structural plasticity (Fawcett, 2009) after spinal cord damage. These principles have been applied in the retina with encouraging results with several studies demonstrating that co-treatment with ChABC at the time of transplantation leads to significant increases in the number of integrated cells (Barber et al., 2013; Ma et al., 2011; Suzuki et al., 2007).

Another major consideration for cell replacement is the long-term survival of the integrated cells in a foreign environment. The eye is frequently described as being immune-privileged. However, photoreceptor cell death is associated with activation of the resident macrophage population, called microglia. The presence of increased numbers of macrophages shortly after cell transplantation is associated with significantly fewer integrated photoreceptors in wild type mice (Warre-Cornish et al., 2014; West et al., 2010). Immune privilege is further compromised if RPE cells die or the outer blood-retinal barrier is disrupted (Wenkel and Streilein, 1998), as often occurs in degenerating or injured retinae. The transplantation procedure itself introduces further, albeit localised, trauma. Together, these have significant implications for photoreceptor transplantation. A number of tissue sources are under consideration for photoreceptor replacement therapy, including ESCs, induced Pluripotent Stem Cells (iPSCs), and dissociated cells either from acute tissue isolation or from cultured populations (see below), as well as the transplantation of whole retinal sheets (Seiler and Aramant, 2012). Many of these will have an immune profile different to the cells of the recipient retina.

Few studies have examined the survival of retinal cells transplanted to the subretinal space, although a number of studies have examined the long-term survival of neural stem/progenitor cells. Interestingly, cultured neural progenitors appear to be less immunogeneic, compared with freshly dissociated neural progenitors, even following xenotransplantation (Hori et al., 2003; Ma and Streilein, 1998). Similarly, others have demonstrated good survival of cultured retinal allografts in pigs at 10 weeks posttransplantation (Warfvinge et al., 2006). The survival of the transplanted cells in these studies was evaluated by examining the mass of cells present in the subretinal space (Warfvinge et al., 2006; Wojciechowski et al., 2002). By transplanting post-mitotic photoreceptor precursor cells, we further sought to assess the ability of integrated donor 
photoreceptors to survive within recipient retinas with partially mismatched haplotypes. We found that transplanted photoreceptor cells integrated within the adult mouse retina are subject to a delayed host immune response from around four months post-transplantation. However, they can survive for extended periods of time, up to a year, provided these immune responses are modulated (West et al., 2010). These are promising results but do demonstrate that the host immune system must be added to the factors to consider when assessing the relative merits of the different donor cell types.

Another major challenge, and the one that is discussed in detail in sections $7 \& 8$ (also summarised in Table 1), is the scalable generation of donor cells suitable for transplantation. The studies outlined in section 6.1 indicate that donor cells need to be at an appropriate developmental stage, post-mitotic precursors, at the time of transplantation for optimal integration. However, photoreceptor genesis in humans occurs between weeks 11 and 16 (O'Brien et al., 2003). This presents not only an ethical but also a practical problem; it is not possible to acquire sufficient donor cell material from developing embryos and we thus require an alternative donor cell source. Stem cells themselves appear to have limited potential to generate new photoreceptors when directly transplanted. However, if they can be directed towards the photoreceptor precursor stage in vitro, they should, in theory, have the potential for effective transplantation when placed in vivo. This is a formidable challenge, but by using the wealth of knowledge surrounding the intrinsic and extrinsic factors required for proper retinal development and regeneration, researchers are now taking major steps towards achieving it.

\section{Directed Differentiation of Ocular Stem/Progenitor Cells to Generate Transplantation-Competent Donor Cells}

Given the at least partial regenerative capacity of the eye itself, there has been considerable interest in the use of ocular stem cell populations as a source for the targeted generation of donor photoreceptors for transplantation. Below we consider the progress made and our current understanding of the potential of the different ocular structures as a source of donor photoreceptors for transplantation (Figure 1b and summarised in Table 1). 


\subsection{De-Differentiation of Pigmented Epithelial Cells}

Pigmented epithelial cells of the mammalian CE, RPE and iris have generated much interest in the field retinal stem cell biology and cell transplantation therapies for retinal degenerative diseases as a potential source of donor cells. They are embryonically related to the neural retina and can be obtained relatively easily through surgery, meaning that they could be obtained from the patient (i.e. they are autologous). While only limited regeneration potential has been documented in vivo (section 5), in vitro studies have demonstrated that these cells can be expanded in culture.

\subsubsection{Ciliary Epithelium-Derived Stem Cells}

Although the mammalian adult retina appears to have restricted regenerative capabilities (section 5.1.2), Van der Kooy and Ahmad independently reported that the mammalian CE contains a population of retinal stem cells (Ahmad et al., 2000; Tropepe et al., 2000). Dispersed throughout the pigmented epithelium, these proposed retinal stem cells represent a very rare population (approximately $0.2 \%$ of CE pigmented cells), and under normal conditions remain quiescent during adult life. However, when isolated from the eye and cultured in vitro, these cells demonstrate characteristics typical of neural stem cells, including self-renewal and multipotency. With or without exogenous growth factor stimulation (typically FGF2 and/or EGF), these single pigmented cells clonally proliferate to form sphere colonies containing similar proportions of pigmented and non-pigmented cells. Despite their heavy pigmentation, non-pigmented colony-forming stem cells can be derived from albino animals, suggesting that the biochemical properties of pigment formation are not required for stem cell function (Ahmad et al., 2000; Tropepe et al., 2000). While initially identified in rodents, these cells have subsequently been isolated from rabbit (Inoue et al., 2005), pig (Gu et al., 2007; Macneil et al., 2007), monkey (Martinez-Navarrete et al., 2008) and human (Bhatia et al., 2009; Coles et al., 2004; Jasty et al., 2012; Martinez-Navarrete et al., 2008; Mayer et al., 2005; Moe et al., 2009; Xu et al., 2007a) eyes, suggesting an evolutionary conservation of this population in the adult vertebrate eye.

Demonstrating a cardinal feature of stem cells, the resulting neurospheres can be dissociated and grown after several passages, though many reports have suggested that the growth of these cells as free-forming spheres become less vigorous after multiple passages, especially when compared to brain-derived neural stem cells (Moe et al., 2009; Xu et al., 2007a; Yanagi et al., 2006). However, these cells have a broader expansion potential when 
cultured as monolayers, maintaining a progenitor phenotype (Coles et al., 2004; Gualdoni et al., 2010; Macneil et al., 2007; Xu et al., 2007a). Comparing mouse, rat and human derived CB retinal stem/progenitor cells, human-derived $C B$ cells formed similar numbers of neurospheres colonies after one week in culture; however the average numbers of cells within each sphere was substantially less than in rodents. This could reflect the differences in development between the species, or could represent a more limited and restricted stem cell potential through the course of evolution (Xu et al., 2007a).

These cells are also capable of responding to a number of exogenous signalling molecules in vitro; increased proliferation of these cells has been documented following supplementation with (i) SCF (stem cell factor), which is believed to act via the c-Kit receptor (Das et al., 2004), (ii) Notch signalling as observed through the upregulation of the Notch1 receptor (Das et al., 2004), as well as (iii) the canonical Wnt pathway mediated through Wnt3a (Das et al., 2006b). More recently, Balenci and colleagues demonstrated that the proliferative behaviour of these adult retinal stem/progenitor cells in vitro reduces when exposed to members of the BMPs, specifically BMP2 and BMP4 (Balenci et al., 2013). In this screening, secreted Frizzled-related protein 2 (sFRP2) also reduced the numbers of adult retinal stem cell primary spheres. Interestingly, the authors noted that these factors are secreted by the adult mouse cornea and lens, implying that the continued quiescence of these cells in vivo in the face of damage or retinal degeneration (see section 5.1.2) may be due, at least in part, to their inhibition by the secretion of a combination of factors by surrounding tissues (Balenci et al., 2013).

Several teams have tried to expand and subsequently differentiate CE-derived progenitor cells (Ahmad et al., 2000; Coles et al., 2004; Das et al., 2005; Tropepe et al., 2000; Xu et al., 2007a). After several days in culture, these cells express early neuronal markers, such as Nestin and Vsx2, indicative of differentiation into a neural cell lineage. Transcriptome profiling of CE-derived stem/progenitor cells demonstrates that they share $80 \%$ of genes with early retinal progenitor cells, but considerably fewer with late retinal progenitors (Das et al., 2005). Similarly, these cells express the FGF receptor, FGFR1, when cultured without the presence of exogenous GF stimulation, as do early progenitors in vivo (Giordano et al., 2007). Das et al. reported that these CE-derived cells preferentially produce earlier born retinal cells in vitro (Das et al., 2005), although others have reported their differentiation into later born cell types, including rods and bipolar cells. There remains some uncertainty in the field as to whether or not CE-derived progenitor cells are truly multipotent and thus fulfil an important criterion of true stem cells (Cicero et al., 2009; Gualdoni et al., 2010; Moe et al., 2009; Yanagi et al., 2006). A number of reports found expression of the rod photoreceptor 
marker, Rhodopsin, following differentiation (Asami et al., 2007; Coles et al., 2004; Tropepe et al., 2000), although other studies have failed to convincingly detect this marker by immunohistochemistry (Cicero et al., 2009; Gualdoni et al., 2010; Macneil et al., 2007). Using the Nrl.GFP transgenic reporter line that expresses GFP in the developing and mature rod photoreceptors, Gualdoni et al. found that the rod differentiation cascade was not initiated in the CE-derived retinal stem/progenitor cells in vitro when using a number of standard differentiation conditions previously reported to generate rod photoreceptors. They demonstrated that these CE cells do possess the ability to proliferate in culture, concomitantly losing their pigmentation over time and expressing a subset of eye field and retinal progenitor markers. However, they also maintained expression of some markers of differentiated CE and typically lacked a neural morphology (Gualdoni et al., 2010).

This study supports previous work in which Cicero and colleagues proposed that both murine and human CE-derived neurospheres consisted of proliferating pigmented epithelial cells rather than true retinal stem cells or progenitors. Dyer, Cicero and colleagues used gene expression studies to show that these cells express markers of the mature CE, rather than genes normally expressed in retinal progenitor cells or stem cells. Lastly, the authors showed that a subset of these cells, approximately 17\%, became Nestin-positive but that expression was not sustained, and the cells failed to adopt a mature retinal phenotype. Collectively, these results challenge the idea of a true retinal stem cell population within the mammalian $\mathrm{CB}$, instead supporting the notion of partial CE transdifferentiation. However, they do not rule out the existence of a rare dormant stem cell. The fact that only a very small proportion of CE cells are capable of giving rise to retinal neurospheres in vitro argues against CE transdifferentiation. More recent studies have demonstrated that these sphereinitiating cells can be prospectively enriched based upon size, pigmentation density and the low expression of $\mathrm{P}$-cadherin as well as high Pax6 expression (Ballios et al., 2012; Demontis et al., 2012; Inoue et al., 2010; Xu et al., 2007b). When sorted on the basis of size and pigmentation criteria, Ballios et al. reported that this population is more efficient at generating spheres than other cells within the CE (Ballios et al., 2012). Moreover, when cultured in the presence of factors required for rod photoreceptor maturation, including taurine, RA, Shh and FGF2 (see section 4.2.1; Figure 2b), Rhodopsin expression was increased, and when cultured for extended periods of time (up to 44 days), these cells lost their pigmentation and ciliation, and adopted a retinal morphology, indistinguishable from cultured primary photoreceptor precursors (Ballios et al., 2012; Demontis et al., 2012). 
It may be premature to reach a final conclusion on this topic based purely upon sequential marker expression and in vitro morphological analyses alone. Like many protocols to differentiate other pluripotent stem cell types (see section 8.1), these cells do not form outer segments in vitro (Ballios et al., 2012). Whether they can be induced to do so using the newly described $3 D$ culture techniques (see section 8.1 ) remains to be seen and a full characterisation, including functional studies, of the progeny of these CE-derived cells is still required. Nonetheless, three independent studies, on both mouse and human CE-derived cells, have shown these newly differentiated cells are functionally responsive, as demonstrated through the acquisition of electrophysiological characteristics (Del Debbio et al., 2013; Demontis et al., 2012; Jasty et al., 2012). In one instance, using the AAV2/8-pRho-eGFP construct, Demontis et al. demonstrated that single eGFP-positive cells expressed cGMP channels gated by endogenous cGMP, as well as voltage-gated channels, typical of rod maturation. Furthermore, they showed that these cells displayed rudimentary responses to light reminiscent of P10 rats and developing rods in Xenopus tadpoles, whose light responses are of smaller amplitude and lower sensitivity than those of their adult counterparts (Demontis et al., 2012).

The debate over the identity and multipotency of CE cells highlights an important issue with many studies attempting to characterise multipotency, particularly the heavy dependency on the expression of single markers, such as Rhodopsin, for the assessment of retinal neuronal potential. Moreover, many studies assess the expression any given marker by just one technique, typically immunohistochemistry. These may lead to erroneous conclusions both about the potential of these cells, and the culture conditions that generate them. Such problems stand to be addressed by the increasingly elegant studies that use transgenic and viral reporter lines. To summarise, it remains possible that CE-derived cells could be harnessed for clinical cell transplantation once a number of obstacles associated with lineage conversion and cell number expansion are overcome.

\subsubsection{RPE-Derived Stem Cells}

Initial studies monitoring the in vitro potential of RPE-derived cells reported that these cells lacked the ability to generate neurospheres in vitro from both mouse and human RPE tissues (Coles et al., 2004; Tropepe et al., 2000). However, following subsequent modifications designed to improve the clonal growth and expansion of adult neural stem cells in vitro (Wachs et al., 2003), Engelhardt and colleagues were able to demonstrate that rat RPE cells, when dissociated and cultured in vitro, express the neural progenitor markers, Nestin and Musashi1, while down-regulating RPE-specific markers, including RPE65 
(Engelhardt et al., 2005). Similar to the CB, these cells are able to proliferate in vitro under both adherent and neurosphere conditions, although expansion was more limited. A low proportion of these cells were able to differentiate in vitro into neuronal cells, as determined through the expression of the neuronal marker $\beta$-III tubulin, and Doublecortin, a specific marker for neuronal precursors (Lee et al., 2003), and the adoption of a neuronal morphology. Despite this, further cultivation of these cells under these differentiating conditions (removal of growth factors and addition of $5 \%$ serum) failed to give rise to a more mature retinal phenotype, indicating that, at least under the culture conditions used within this study, RPE cells are capable of de-differentiating into a young neuronal phenotype, but lack the capacity of retinal maturation (Engelhardt et al., 2005).

Promisingly, more recent work by Temple and colleagues has shown for the first time that RPE tissue derived from human adult donors can be successfully coaxed into a multipotent state (Salero et al., 2012). These cells are able to undergo several rounds of division (doubling approximately every two days) when cultured under adherent conditions, prior to differentiation into multiple lineages, including CNS and mesoderm-associated lineages. Under these conditions, proliferation was stimulated by serum alone, and the cells could be passaged at least six-eight times. Surprisingly, RPE cells harvested from 99-year-old donors were also shown to produce actively growing cultures, similar to the growth of RPE cells derived from younger donors, suggesting that donor age is not an impediment to expanding these cells in vitro. When grown under clonal, non-adherent conditions, these cells form neurospheres in the presence of knockout serum replacement media and FGF2. Primary neurospheres were visible after four days in vitro and could be passaged at least three times. While these dissociated RPE cells were able to form neurospheres in culture, it was noted that these cells grew more readily when plated under adherent conditions, most likely reflecting the importance of cell-cell interactions and cell adhesion for RPE growth.

Of value for transplantation, RPE-derived progenitor cells are capable of fate change when grown in conditions to promote retinal differentiation, which included the addition of Fgf2, Fgf8, Shh and RA. Under these conditions, gene expression analyses demonstrated that these cells are able to upregulate the expression of Nestin by 1000 -fold, and $\beta$-tubulin III by 90 -fold. Furthermore, these cells expressed markers consistent with the early forebrain/eye field lineage, including LHX2, OTX2, and RAX. However, the expression of more mature retinal markers, including RHODOPSIN and VSX2, did not increase, demonstrating a limited potential to differentiate towards mature retinal neuronal phenotypes under these differentiation conditions. Importantly, it should be noted that these cells were cultured for four weeks, a relatively short time in terms of human development. Those protocols reporting 
a promising photoreceptor-like morphology from murine CE-derived cells were maintained for a culture period of 44 days (Ballios et al., 2012; Demontis et al., 2012; Salero et al., 2012). Collectively, these findings yield hope for the use of RPE cells for autologous or allogenic replacement therapies and a thorough assessment of the differentiation potential of these cells with the use of more sophisticated methods (as outlined in section 8.1) will be important (Salero et al., 2012).

\subsubsection{Iris-Derived Stem Cells}

While reports of iris-derived transdifferentiation have not been documented in vivo, in vitro studies have indicated that a subset of these cells retain the potential to generate neurospheres when removed from their in vivo environment. Neurospheres from these cells can be derived from early postnatal through to adult stages in mouse, rat and human, although the number of neurospheres generated declines with age (Asami et al., 2007; Jasty et al., 2012). Application of exogenous growth factors (FGF2) demonstrates that while neurospheres can form without stimulation, the presence of growth factors results in a 3.5fold increase in neurospheres generation. Furthermore, enrichment of Nestin-positive cells via the use of a transgenic reporter line driving GFP expression from the Nestin promoter (Yamaguchi et al., 2000), results in a more pronounced capability of these cells to form neurospheres. Similarly, enrichment of neurospheres-initiating cells from the IPE is also achievable by Fluorescence-Activated Cell Sorting (FACS) for the cell surface marker CD133, a broad marker associated with both neural and haematopoietic stem cells. Following this selection criterion, a greater number of neurospheres are formed, in fact even greater than the Nestin-positive IPE subpopulation. Further analyses of CD133-positive and negative populations demonstrates that the CD133-positive fraction of cells yields spheres containing more proliferative cells, some of which express Nestin and Musashi1, whereas some of the CD133-negative cells are shown to acquire TuJ and/or Nestin expression, adopting more of a neuronal morphology. The CD133-positive population therefore appears to represent the proliferative population of cells, and as such, could be used for enrichment prior to differentiation in vitro (Asami et al., 2007). Whether CD133 represents a positive selector for stem like cells in other regions, like the CE, is not known.

While capable of proliferating in vitro, IPE-derived cells also possess the ability of transdifferentiating into neuronal cell types, without proliferating. Following culture with FGF2, IPE-derived cells increase mRNA expression of pro-neural genes, including Nestin, Notch, Otx2 and Pax6, while concomitantly undergoing pigment elimination (Asami et al., 2007). Furthermore, prolonged culture in the presence of fetal bovine serum (FBS) induces 
the expression of Rhodopsin (photoreceptor cells), TuJ, Glutamine Synthetase (Müller glia cells) and $\mathrm{HPCl} /$ syntaxin (amacrine cells). Similar to these rodent studies, pigmented porcine iris cells have also been shown to proliferate, to form spheres, and to differentiate into neurons and glia, although retinal-specific markers were not expressed under the conditions used in this study (Macneil et al., 2007). More recently, human iris tissue acquired following iridectomies has been shown to be equally capable of proliferating and expressing neural progenitor markers, including Pax6, Sox2 and Nestin. Again, however, the IPE-derived cells show only partial neuronal differentiation, expressing $\square$-III-tubulin, Map-2 and Rhodopsin, whilst retaining their differentiated epithelial phenotype (Froen et al., 2011).

While differentiation of IPE cells into photoreceptors is rare, it is possible to induce the expression of photoreceptor-specific markers by means of gene transfer (Akagi et al., 2004; Akagi et al., 2005; Haruta et al., 2001). For instance, mis-expression of Crx induces rat IPE cells to express several photoreceptor specific antigens, including Rhodopsin, Recoverin and Arrestin (Akagi et al., 2004; Akagi et al., 2005). Interestingly, in primates, forced expression of both NeuroD and Crx in combination is required to induce a photoreceptor-like phenotype, demonstrating that this approach, although slightly altered from that of rodents, is feasible in higher vertebrates.

\subsection{Müller Glia-Derived Stem Cells}

In keeping with the progenitor-like profile that Müller glia can adopt in lower vertebrates, Müller glia cells of the mature mammalian retina share significant transcriptome overlap with retinal progenitor cells (Blackshaw et al., 2004; Roesch et al., 2008). Given these properties, it has been postulated that these cells could provide a source of retinal neuronal cells within the adult mammalian retina. Confusingly, however, when using the neurosphere assay, Tropepe and colleagues reported no production of neurospheres from the adult central retina. This may mean that the central retina of mature mammals lacks the same selfrenewal properties demonstrated in lower vertebrates, and/or may not be able to respond to the neurosphere culture conditions. In line with the latter, Das et al., demonstrated that Müller glia cells obtained from adult rats can be cultured under proliferative conditions before being transferred to neurosphere-generating conditions. These conditions resulted in a small subset $(0.18 \%)$ of retrospectively enriched Müller glia cells that can generate clonal neurospheres (Das et al., 2006a). These Müller glia-derived neurospheres express neural stem cell markers, including Sox2, Nestin and Musashi1, and are able to generate secondary and tertiary spheres, demonstrating their ability to self-renew. Furthermore, when 
induced to differentiate through the removal of FGF2 and the addition of serum, these cells are able to change their morphology adopting more of a neuronal phenotype, as well as giving rise to both neurons and glia. Finally, when dissociated and transplanted in vivo, these cells migrate into the recipient retina although the evidence to indicate that they form new photoreceptors is limited.

Components of the Wnt and Notch signalling pathways are upregulated in Müller glia enriched cultures derived from the normal adult rat retinas (Das et al., 2006a). In keeping with this, exogenous supplementation with Wnt2a significantly increases the generation of neurospheres from these cells, whereas there is a notable decrease when cultured with the Wnt antagonist, Fzd-CRD. Likewise, when enriched Müller glia cultures are transfected with NICD to constitutively activate the Notch pathway, there is a significant increase in neurosphere production (Das et al., 2006a). Interestingly, the presence of antagonists of either the Wnt or the Notch signalling pathway results in a lower number of neurospheres than controls, implying that endogenous Wnt and Notch signalling is required for the maintenance of stem cell properties of these enriched Müller glia cells (Das et al., 2006a). Inactivation of the tumour suppressor gene $p 53$ significantly increases the proliferation of these cells, believed to be due to the nuclear accumulation of $\square$-catenin and Yap protein in the $p 53^{-/}$Müller glia-derived retinal progenitors (Zhao et al., 2014). Importantly, and of relevance for transplantation studies, when these cells are transplanted into host animals, they can integrate and express either retinal ganglion cell or photoreceptor markers, such as Islet1 and Brn3, or Rhodopsin and IRBP, respectively. Furthermore, despite the lack of the tumour suppressor gene, these cells do not generate tumours, aligning with the fact that there are no documented cases of tumours of Müller glia cell origin (Zhao et al., 2014).

A population of Müller glia cells with stem cell characteristics has been isolated from the adult human retinae, suggesting that this regenerative capacity may be utilised in response to injury or disease (Bhatia et al., 2009; Lawrence et al., 2007). While there is no evidence to currently suggest that they can contribute to regeneration in vivo, they can be induced to grow and differentiate in vitro. In a recent study, Singhal and colleagues transplanted retinal ganglion cells derived from human Müller glia cells into the eyes of Lister hooded rats previously depleted of retinal ganglion cells by NMDA and reported an improvement of the negative scotopic threshold response of an ERG (indicative of RGC function) (Singhal et al., 2012). Subsequently, it has been shown that it is also possible to generate photoreceptorlike cells from human Müller glia cells. When transplanted into $\mathrm{P} 23 \mathrm{H}$ rat, a rodent model for primary photoreceptor degeneration, these cells are able to migrate and integrate into the retina, and potentially improve photoreceptor function in vivo, as indicated by an increase in 
a-wave amplitude and slope of the scotopic flash ERG (Jayaram et al., 2014) These results suggest that human Müller glia stem cells isolated from the normal adult retina might be cultured in vitro to generate a source of retinal cell types suitable for transplantation studies. However, a challenge for this source is that it is unlikely to be any more plentiful than those from human embryonic sources, necessitating highly efficient expansion strategies and potentially limiting their use as a donor source for transplantation.

\subsection{Overview of Ocular-Derived Donor Cells - Limitations}

The discussion above shows that there exists in the literature a wide array of protocols aimed at generating specific retinal cells types, typically photoreceptors, from ocular stem cell-like sources. As we move towards more effective differentiation protocols, such as those described in the next section, these cells still represent a viable source for autologous transplantation. There remain a number of issues to resolve, however. These include whether such sources can generate donor cells in the numbers required for effective transplantation. Moreover, it is not yet known how faithfully these newly generated cells reflect their new phenotype, whether they retain aspects of their initial identity or how stable their newly acquired identity is over time. Further studies are also required to verify the true functionality of these cells, as well as their integration and survival capabilities in vivo.

\section{Directed Differentiation of Pluripotent Stem Cells to Generate Transplantation- Competent Donor Cells}

Pluripotent stem cells are an attractive alternative to ocular-derived stem cell populations as a source of donor cells (Figure 1c-d). Although ineffective for photoreceptor transplantation in their undifferentiated state, they represent a potentially inexhaustible source of donors, if we are able to fully recapitulate retinal development in vitro to a developmental state equivalent to the period of photoreceptor genesis. In the early 1980's, two independent laboratories published reports describing the isolation of pluripotent ESC lines from the inner cell mass of murine blastocysts (Evans and Kaufman, 1981; Martin, 1981) (Figure 1d). ESCs are characterised by a number of key properties. They can be maintained indefinitely in an undifferentiated state in vitro, demonstrate the capacity to differentiate into cells from all three germ layers (endoderm, mesoderm and ectoderm) and following their introduction into the pre-implantation stage embryo, they give rise to chimeric animals. Fifteen years later, human embryonic stem cells (hESCs) had also been isolated (Thomson et al., 1998), initiating a wave of interest in ESCs as a possible source for cell replacement therapies. 
The development of iPSCs technology represents a new autologous source of cells (Figure 1c). Importantly, iPSCs also offer a new source of human cells for studying human development, physiology and a number of pathologies. In 2007, Takahashi and Yamanaka reported that, from a screening of 24 different factors, just four transcription factors (Oct4, Sox2, c-Myc and Klf4) are necessary to reprogram human fibroblasts into iPSCs. These cells possess many features similar to ESCs, including morphology, self-renewal ability and the capacity to form teratomas (Takahashi et al., 2007). Since then, iPSC technology has evolved and there are now many reports of iPSCs that are similar, albeit not identical, to ESCs, not only in gene expression but also in miRNA expression, DNA methylation and histone modification patterns. Importantly, iPS cell lines capable of generating adult chimeras with germ line transmission have been described (Gai et al., 2009; Hamanaka et al., 2011; Maherali et al., 2007; Okita et al., 2007; Wernig et al., 2007). Moreover, somatic cells can now be reprogrammed into iPSCs using a variety of approaches, including integration-free methods, such as Sendai virus, episomal plasmids, recombinant proteins (Kim et al., 2009) and small molecules [(Zhou et al., 2009); see review (Borooah et al., 2013)].

Fibroblasts are the most common source of cells for reprogramming, although a number of tissues can now be utilised to derive iPS cell lines, including blood (Loh et al., 2009), keratinocytes (Aasen et al., 2008), cord blood cells (Giorgetti et al., 2009), and even adult neural stem cells (Kim, 2007). Furthermore, the differentiation status, i.e. the epigenetic state of the somatic cells chosen, appears to influence not only the reprogramming efficiency but also the potential of the cells to differentiate into the desired cell type for therapy (Kim et al., 2010). Hematopoietic stem cells, for example, are reprogrammed into iPSCs much more efficiently than fully differentiated lymphocytes (Eminli et al., 2009). In the eye, foetal RPE has also been reprogrammed into iPS cell lines. When compared to other iPS and ES cell lines for their capacity to generate RPE colonies in vitro following differentiation, the human foetal RPE-derived iPS cell lines have been shown to perform superiorly (Hu et al., 2010).

The success of future cell replacement therapies will likely depend on the right choice of the initial pluripotent population and the ability to efficiently guide these cells towards the required differentiated cell type. The generation of reliable and robust differentiation protocols that can be used with either ES or iPS cell lines is, therefore, of great importance. There has been very significant progress to this end over the last 10 years, which we discuss below. 


\subsection{Obtaining Retinal Cells from Pluripotent Stem Cells}

Evans and Martin both demonstrated that upon exposure to serum, floating embryoid body (EB) cultures of ESCs undergo differentiation and generate many cells types from the three germinal layers. However in these studies, the presence of neural tissues was not pronounced (Evans and Kaufman, 1981; Martin, 1981). Since then, there has been intense investigation into protocols for the generation of neural lineages from mouse ESCs (Bain et al., 1995; Kawasaki et al., 2000; Lee et al., 2000; Mizuseki et al., 2003; Wichterle et al., 2002; Ying et al., 2003). To date, there are two main methods for the differentiation of pluripotent stem cells into multi-lineages. The first protocol encompasses ESC differentiation in a monolayer (2D method) culture, while the second approach relies on the formation of cell aggregates, or EBs, grown in suspension. The differentiation potential into different cell fates is further determined by modifications to the culture environment, such as the addition of growth factors, serum concentration, nutrient limitation and ECM (Kurosawa, 2007) (Figure 2c).

As described in section 4.1, in normal development the eye is specified in a stepwise manner, commencing with commitment to a telencephalic identify, followed by formation of the eye field (Figure 2a). One of the first studies to report the generation of ESC-derived retinal cell types was based on methods for neural differentiation. In a study by Zhao et al., neural progenitor differentiation was induced either by the addition of RA or insulintransferrin-selenium fibronectin (ITSFn) and basic FGF (bFGF) to cultures (Zhao et al., 2002). Further differentiation into photoreceptors was achieved by co-culture with postnatal (P1) retinas. This study provided a proof-of-concept for ESC differentiation into cells that express markers of rod photoreceptors.

Subsequently, efficient telencephalic differentiation of mouse ESCs was demonstrated by using a serum-free floating culture of EBs (SFEB) system. Under these conditions, further treatment of SFEB cultures with Wnt and Nodal antagonists, Dickkopf-1 (Dkk-1) and LeftyA (SFEB/DL), respectively, improved the efficiency of Bf1-positive telencephalic cell generation considerably (nine-fold increase over $15 \%$ as opposed to 1.6\%) (Watanabe et al., 2005). During early embryonic patterning, Wnt signalling mediates dorsal-ventral patterning of the embryo, whilst Nodal signalling is involved in both anterior-posterior and left-right patterning. Specifically, Dkk-1 is a secreted protein, which has been shown to be necessary for head induction (Glinka et al., 1998) and Lefty is understood to be important in regulating the range and duration of Nodal activity (Sakuma et al., 2002). As such, the combination of both factors was sufficient to generate ESC-derived neural and rostral populations. 
Finally, further differentiation of these cells towards a Rax/Pax6-positive retinal lineage has been achieved when the SFEB/DL-generated telencephalic EBs were treated with $5 \%$ serum and activin-A (SFEB/DLFA) at initial stages of culture [Figure 2c; (Ikeda et al., 2005)]. In this work, Ikeda and colleagues examined a number of factors known to be important in retinal development, including Shh, Wnt, BMP4, Nodal (without LeftyA), IGF, FGF1, FGF2, and FGF antagonists for their ability to induce Rax-positive retinal progenitors. However, only serum and activin-A were shown to induce significant retinal-directed differentiation. The mechanism of action of these two factors in early eye development has yet to be elucidated. Nevertheless, $\sim 26 \%$ of generated cells expressed markers of RPCs and were able to differentiate into Crx-positive photoreceptor precursors. When cultured with embryonic retinal cells, SFEB/DLFA differentiated ES cells gave rise to a small number Rhodopsin and Recoverin positive cells, able to migrate into retinal explants in vitro. This was the first study to demonstrate the systematic and stepwise in vitro differentiation of ESCs into rostral neuronal progenitors, followed by progression to retinal progenitors, and finally photoreceptor precursors.

Since then, a number of studies have combined the idea of sequential specification, starting with the induction of a telencephalic, followed by retinal, identity. Lamba et al. reported the first differentiation of hESC into RPCs using this concept [Figure 2c; (Lamba et al., 2006)]. In this study the authors employed the Wnt antagonist, Dkk-1, as described above, a BMP antagonist, Noggin, and IGF-1. During normal stages of development, both Noggin and Dkk1 act as neural inducers (Smith and Harland, 1992). IGF-1 is another head inducer factor, which functions in a manner similar to Dkk-1 by antagonising Wnt signalling in the early developing embryo. In Xenopus, overexpression of IGF-1 leads to the formation of ectopic eyes (Richard-Parpaillon et al., 2002). Following an initial brief suspension culture where neural induction was stimulated, EBs were plated on poly(D-lysine) and Matrigel for further differentiation. The cells were cultured in the continued presence of Dkk-1, Noggin and IGF1 , with the addition of bFGF. Under these conditions, the differentiated cultures contained about $80 \%$ RPCs and $12 \%$ were CRX-positive photoreceptor precursors after three weeks in culture (Lamba et al., 2006). It is worth noting that this is significantly faster than normal human eye development, where the first CRX-positive photoreceptor precursors would not normally be seen until week ten (O'Brien et al., 2003).

The protocol described by Lamba yields a high percentage of RPC-like cells in a relatively short time frame. However, the inclusion of the basement membrane matrix, Matrigel, which contains a number of unspecified growth factors, is likely to influence differentiation and, 
potentially, the duration of differentiation. For ESCs to be used in the clinical setting, it is of paramount importance to have precisely defined culture conditions capable of inducing retinal specification. This was achieved a couple of years later by Osakada et al. This team was the first to describe defined culture conditions that promoted photoreceptor differentiation from mouse, monkey and human ESCs [Figure 2c; (Osakada et al., 2008; Osakada et al., 2009b)]. Their protocol was based on the SFEB/DLFA method described by Ikeda et al., which was used to generate RPCs from a mouse ES cell line where GFP was knocked in at the Rax locus. Rax-positive RPCs were isolated by FACS and further cultured on poly-D-lysine/laminin/fibronectin-coated dishes. Inhibition of Notch signalling has been shown to promote photoreceptor differentiation in vivo (Jadhav et al., 2006; Yaron et al., 2006). Accordingly, Osakada et al. found that the addition of the $y$-secretase inhibitor, DAPT increased the number of Crx-positive photoreceptors precursors. Of note, the addition of DAPT at the early RPC stage, when the retinal progenitors were still dividing, led to a decrease in the number of mitotic cells and a concomitant increase in early born ganglion cells and Crx-positive photoreceptor precursors. All other retinal cells, including horizontal, amacrine, bipolar, immature Müller cells and immature RPE, were present in these cultures, with no significant changes in their numbers or ratios following DAPT treatment. Therefore, DAPT appears to direct the mitotic RPCs toward differentiation to post-mitotic photoreceptor precursors and ganglion cells. To further improve photoreceptor differentiation, the addition of a cocktail of factors (acidic FGF, basic FGF, taurine, Shh and RA) known to be involved in rod genesis during development, was assessed. In agreement with the developmental studies, the presence of these extrinsic factors enhanced rod photoreceptor differentiation with approximately $17 \%$ of total cells expressing the photopigment Rhodopsin.

One of the most elegant and comprehensive descriptions of the stages of retinal differentiation arising from human ES and iPSCs is that of Meyer et al. (Meyer et al., 2009). In this study, a combination of suspension and adherent two-dimensional (2D) cultures was used. A striking difference with this, compared to previous protocols, is that no inducible factors were added to the suspension culture and retinal differentiation was, instead, achieved by endogenous secretion of Dkk-1, Noggin and FGF signalling. Following a period in suspension, cell aggregates were plated on laminin and allowed to form neuroepithelial rosettes, which were then mechanically isolated to grow as suspension structures again. This combination of suspension/2D-adherent culture conditions proved to be very effective in producing RPCs and CRX-positive photoreceptor precursors in a temporal order that closely mimics normal development. Further improvement of this protocol permitted the differentiation of human ESC and iPSC-derived optic vesicles (OV-structures), which could be separated from neural forebrain-like regions [Figure 2c; (Meyer et al., 2011)]. Following 
separation, the OV-structures continued to mature and differentiate and by day 80 of culture, photoreceptors expressed CRX and RECOVERIN. Of worth, these studies did not demonstrate the presence of more mature RHODOPSIN-positive photoreceptors, questioning whether further modifications to these protocols are required.

The most striking and efficient protocol for the differentiation of retinal tissue was developed by Sasai and colleagues [Figure 2c; (Eiraku et al., 2011)]. Using a three-dimensional (3D) culture system, mouse ESCs prepared in the previously described SFEB system form EBs, which are then cultured in suspension with Matrigel (growth factor reduced [GFR]) from day 1 of culture. These conditions permitted the spontaneous formation of self-organised retinal neuroepithelia, which remarkably, evaginates into optic vesicle-like structures expressing the retinal specific progenitor marker, Rax, that in turn invaginates to form bilayered optic cuplike structures during the first week of culture. This was the first time that retinal development, including morphogenesis, had been replicated in vitro from ESCs. Most remarkably, and contrary to what would have been predicted from our understanding of retinal development in vivo, this process occurs in the absence of neighbouring tissues, such as surface ectoderm, lens and mesenchymal tissue. Consequently, Eiraku et al. propose that the signals and physical forces drive retinal differentiation are intrinsic to these cells and the neuroepithelium being formed.

Further retinal development was achieved when the optic cup-like structures were manually isolated from the floating EBs (Eiraku et al., 2011). The isolated optic cups spontaneously formed large, continuous epithelial structures, which showed a clear apical-basal stratification similar to the early postnatal retina. Following a further two weeks of differentiation, all the cell types of the mature retina were detected, with photoreceptors present in the outermost layer (facing the media environment) and other cell types overlying them towards the basal surface of the neuroepithelium (facing the inside of the vesicle). In this study, the only external inductive signals introduced into this culture system came from a short pulse of RA and taurine added to the differentiation culture from days 10 to 14 . RA appears to be very important in maintaining the integrity of the epithelium, since the absence of RA in these cultures led to the loss of lamination, with only neural rosette-like structures remaining. This study represents a landmark in the generation of neural tissues from ESCs. Nonetheless, the conditions still require improvement; there is a loss of neural integrity after 35 days in culture, and these conditions are unable to support the full maturation of photoreceptors, particularly the formation of outer segments. 
Of clinical relevance, a very similar 3D-based differentiation protocol using human ESCs, has been described by the same group [Figure 2c; (Nakano et al., 2012)]. Similar to the mouse ESC differentiation cultures, these cultures were also dependent on GFR-Matrigel and generated optic cup-like structures that self-organised into a stratified neural retina containing all retinal cells, including cone and rod photoreceptors. In contrast to mouse ESCs, a higher concentration of knock-out serum (KSR) was required to derive human retinal epithelium. However, this higher concentration of KSR also triggered an undesired caudalisation of neural progenitors. To solve the problem and drive differentiation to a more rostral phenotype, a Wnt inhibitor, IWR1e, was added together with the ROCK inhibitor, Y27632 , from day 0 of culture. The number of RAX-positive retinal progenitors was further improved when serum and the Hh agonist smoothened agonist, SAG, were added to early stages of culture. By day 42, CRX-positive photoreceptor precursors were present in the neuroepithelium region and 20 days later these cells started to express RECOVERIN. By day 126 in culture, the authors report the expression of markers characteristic of more mature photoreceptors, including NRL and RHODOPSIN. A comprehensive time-course of photoreceptor genesis was not performed in this study, so it is not clear when the onset of this expression occurred. Importantly, and in keeping with the mouse ESC 3D cultures described by the same group, no outer segment formation was observed despite the extended periods of culture.

Over the same period, a variety of retinal differentiation protocols for mouse and human iPS cells have also been described (Hirami et al., 2009; Homma et al., 2013; Jin et al., 2012; Lamba et al., 2010; Mellough et al., 2012; Meyer et al., 2009; Osakada et al., 2009a; Osakada et al., 2009b; Parameswaran et al., 2010; Phillips et al., 2012; Reichman et al., 2014). Hirami and colleagues described one of the first protocols for photoreceptor differentiation from mouse and human iPS (Hirami et al., 2009). A 2D protocol similar to those described for ESCs was used, and included Wnt and Nodal inhibition, supplemented in parallel with RA and taurine. This was sufficient to drive mouse iPS cells towards a photoreceptor fate. Hirami et al., and others, have also described the differentiation of human iPS cells towards this fate, although efficiency is typically lower than that achieved with mouse iPS cells (Hirami et al., 2009; Lamba et al., 2010; Osakada et al., 2009a). Phillips et al. (Phillips et al., 2012) generated a human iPS cell line from reprogrammed blood cells and demonstrated the formation of retinal OV-structures using the Meyer protocols (Meyer et al., 2009; Meyer et al., 2011). Although the retinal epithelium contained RECOVERIN-positive photoreceptors by around day 70 of culture, the presence of late markers, such as OPSINS, were only detected when these vesicles were dissociated and the cells plated on laminin for further culture. Additionally, our group has demonstrated the 
differentiation of human ESC and iPS cells into RPCs and photoreceptor precursors using a Matrigel-based adherent culture system where neuroepithelia-containing vesicles form very early in culture (day 2). Under these conditions, the integrity of the neuroepithelium was lost very quickly, although markers of photoreceptor precursors, such as CRX and NRL, were detectable 30 days after plating (Boucherie et al., 2013).

Recently, Zhong et al. (Zhong et al., 2014) reported further adaptations of the Meyer protocols (Meyer et al., 2009; Meyer et al., 2011; Phillips et al., 2012) and demonstrated that human iPS cell lines can efficiently form neuroepithelium containing retinal structures. Again, this protocol consisted of a stepwise 2D-suspension/3D-suspension differentiation system that is not dependent on exogenous factors at the initial suspension phase; although it does require the addition of GFR-Matrigel (Figure 2c). This study demonstrates for the first time the long-term (up to 180 days) culture of intact retinal neuroepithelial morphology. This appears to be dependent on the presence of serum, taurine and RA. Most importantly, photoreceptor maturation was improved by the addition of RA to these cultures during a specific time window (week 10 to 14 of culture). Furthermore, convincing evidence from electron microscopy images of the mature neuroepithelia regions showed the presence of developing outer-segment discs, albeit in low numbers. In agreement, perforated-patch clamp experiments demonstrated that a couple of photoreceptor cells, tested between week 25 and 27 of culture, were responsive to light. Collectively, this was the first study to demonstrate outer segment formation in vitro. Of note, these structures formed despite the RPE in close apposition to the nascent photoreceptor layer, questioning the whether the proximity of the RPE is truly necessary for photoreceptor maturation. Most recently, Reichman et al. described a very simple differentiation protocol that allows human iPS cells to overgrow under neuralising conditions and both RPE and neuroepithelia vesicles containing photoreceptors were observed after three weeks in culture (Reichman et al., 2014).

The differentiation of mouse and human pluripotent stem cells towards photoreceptor lineages has progressed rapidly in the last decade. A robust number of photoreceptor precursors as well as a small number of more mature and functional photoreceptors can now be obtained from human stem cells using a variety of step-wise differentiation protocols. This includes a number that take significant steps towards clinical application, reducing the need for serum and factors with unspecified content, such as Matrigel. It is important to emphasise the long time required to generate photoreceptors with the more robust and efficient differentiation protocols available. Further improvements, which aim to accelerate the differentiation of photoreceptors, are likely to be necessary for clinical approaches. An 
additional consideration, which is perhaps more pressing, is the need for a careful characterisation of the stages of development of these human ESC-derived photoreceptors, as we know from mice studies that only stage-specific photoreceptor precursors are capable of integrating into the adult retina efficiently (see section 6.1.). Whether or not the same holds true for human photoreceptors remains to be determined.

\subsection{Transplantation of Embryonic and Induced Pluripotent Stem Cell-Derived Photoreceptors}

In the previous section, we described recent advances in generating pluripotent stem cells (PSC)-derived photoreceptors and conclude that we can now generate sufficient numbers of photoreceptors for transplantation experiments. To date, only a relatively small number of studies have transplanted ESC-derived retinal cell types (Banin et al., 2006; GonzalezCordero et al., 2013; Lamba et al., 2009; Lamba et al., 2010; Tucker et al., 2011; West et al., 2012; Zhou et al., 2011). The large body of work on transplantation using early postnatal cells from mice has established a number of parameters required to increase the number of integrated cells following transplantation; these include both the number and purity of the transplanted population (Table 1).

One of the first transplantations of ESC-derived-retinal cells was performed with cells derived from hESCs. Following three weeks of hESCs retinal differentiation (Lamba et al., 2006), Lamba et al. (Lamba et al., 2009) transplanted a virally labelled GFP-positive mixed population of retinal cell types (between $50,000-80,000$ ) to the subretinal space of adult wild type and $\mathrm{Crx}^{-/-}$mice, a model of retinal degeneration that lacks both rod and cone function (Furukawa et al., 1999). Within this mixed population, the differentiated cells were reported to contain $80 \%$ retinal cells, of which $30 \%$ were CRX-positive and $15 \%$ were NRL-positive, indicating that at least a small proportion were at a stage equivalent to post-mitotic rod precursors in the developing retina. Following transplantation into wild type and $\mathrm{Cr} \mathrm{x}^{-/}$retinas, GFP-positive cells were observed in all layers of the retina and a small number of cells were correctly located in the $\mathrm{ONL}$. In the $\mathrm{Crx}^{-/}$animals, donor cell identity was confirmed using a human-specific NRL antibody and an average of 3,000 cells located within the ONL were counted per eye. These cells did not appear to form new outer segments. This may not be surprising since donor cells frequently adopt an atypical morphology in severely degenerated retinas (Barber et al., 2013). Lamba et al. assessed connectivity and functionality of the transplanted cells into the endogenous circuitry by ERG. Despite the low number of 
integrated photoreceptors, the transplanted $\mathrm{Crx}^{-/}$eyes showed a light response with b-wave restoration (Lamba et al., 2009).

Another study by the same group demonstrated the differentiation of a human iPS cell line towards photoreceptor cell fate. Approximately $12 \%$ of the differentiated cells were CRXpositive, with $1 \%$ of all cells in culture expressing late postnatal markers, RECOVERIN and RHODOPSIN. In order to purify and isolate photoreceptors only, the cells were labelled and FAC sorted for the human photoreceptor specific promoter IRBP driving GFP (IRBP-GFP), introduced by lentiviral vectors. Following transplantation into adult wild type eyes, approximately 50 cells per eye were shown to migrate into the ONL. However, no inner processes projecting towards the INL, or inner and outer segments were present (Lamba et al., 2010).

A mouse DsRed iPS cell line has also been used to differentiate photoreceptor precursors with a protocol that used a cocktail of growth factors and recombinant proteins (Tucker et al., 2011). In this study, $\sim 250,000$ retinal cells at day 33 of differentiation were transplanted into the $R h \mathrm{O}^{-/}$, a model that lacks Rhodopsin protein. Importantly, to avoid teratoma formation, these cultured cells were first depleted of cells positive for the SSEA1 marker of undifferentiated ESCs. There, the authors described a repopulation of the ONL following transplantation with an average of 16,000 cells integrated into the ONL. This is particularly striking since transplantation of post-mitotic rod precursors from the postnatal retina leads to the integration of only 500-1,000 donor cells, three weeks post-transplantation (Barber et al., 2013). The DsRed photoreceptors were positive for Rhodopsin, which is absent in the host photoreceptors, and expressed Recoverin and the outer segment markers, ROM1. Similar to results obtained by Lamba et al., the authors reported a restoration of ERG response posttransplantation. Interestingly, correct morphology does not appear to be a prerequisite for function. A recent report demonstrated that photoreceptors with profound structural deficits, including the absence of fully formed outer segments, are still able to support significant levels of visual function. Moreover, functional read-outs, including ERG amplitudes, can be markedly better than might be predicted from the level of Opsin expression and segment formation (Thompson et al., 2014). Together, these findings point to the functionality of stem cell-derived photoreceptors; however, further work is required to demonstrate whether or not they can improve vision.

We recently reported an adaptation of the Osakada SFEB/DLFA differentiation protocol; this involved forming individual EBs in 96-well plates and establishing the optimal cell density for the generation of Rax-positive retinal progenitors, which consequently led to the generation 
of several different retinal cells types, including photoreceptors (West et al., 2012). Our findings have demonstrated the important role of the differentiation niche; cultures containing only RPCs, as determined by the expression of Rax.GFP, resulted in a greater number of Rhodopsin-positive cells than when RPCs were grown together with other neuronal cell types. Thus, a differentiation culture containing a pure population of RPCs is important for efficient photoreceptor differentiation. This 2D method of differentiation has been shown to generate cell populations in which all the aggregates contain Crx-positive cells (> $65 \%$ of cells were Crx-positive) by day 24 , a finding that has been corroborated by quantitative polymerase chain reaction (qPCR) data. Under these culture conditions, the levels of $\mathrm{Crx}$ transcripts at day 28 were comparable in levels to that of the early postnatal (P0) retina. However, of these Crx-positive photoreceptor precursor cells, only a small proportion stained for the more mature photoreceptor markers, Rhodopsin and Recoverin. Moreover, when an Nrl.RFP ES cell line was used to both determine the precise onset of rod photoreceptor differentiation and allow for the isolation of a pure photoreceptor precursor population for transplantation, we found that there were very few $\mathrm{Nrl}$ expressing cells, despite the presence of many Crx-positive cells. This was further underlined by transplantation experiments. Following transplantation of 200,000 FAC sorted GFP-positive retinal cells, labelled with AAV2/9.CMV.GFP virus, into the subretinal space of adult mice, no integrated cells were observed. Together, these results indicated that the defined SFEB/DLFA method of differentiation is unable to accurately recapitulate retinal development, at least for the correct adoption of photoreceptor fate, nor be scaled up sufficiently to produce large numbers of transplantation competent photoreceptor precursors.

As mentioned above, in 2011, Sasai and colleagues reported the near-normal histogenesis of retinal neuroepithelium using a 3D culture system (Eiraku et al., 2011). In 2013, we reported the first study assessing the transplantation competence of mouse ESC-derived photoreceptors generated using an adapted version of the Eiraku protocol (GonzalezCordero et al., 2013). To scale up the protocol to produce high numbers of photoreceptors for transplantation, the EBs were kept as intact structures (whole EBs), as opposed to the manually isolated optic vesicles described by Eiraku et al. Additionally, serum free culture conditions containing RA and taurine at normal levels of $\mathrm{O}_{2}$ were shown to be more conducive to retinal development, allowing for the formation of inner and outer nuclear layerlike structures containing mature retinal cell types, including photoreceptors. In our study, a precise characterisation of rod genesis in the 3D differentiation cultures established that ESC-derived rods are similar to early postnatal stages of development (with day 26 of cultured cells corresponding to P4-P6 of development). Importantly, this demonstrates that the timing of photoreceptor development in 3D cultures closely follows normal in vivo 
development. Furthermore, the authors demonstrate that ESC-derived rods went on to develop features of mature photoreceptors, as indicated by the presence of late markers of the phototransduction cascade, rod $\square$-transducin and Peripherin. This stage of culture is therefore more similar to the P10-12 stages of development. Finally, it was shown that inner segments and cilium-like structures were observed by electron microscopy, although the formation of outer segment-like structures was not detected. Most recently, Roska, Busskamp and colleagues have recently reported the formation of outer segments, together with inner segments and connecting cilia, and light-evoked electrical responses from mouse ESC-derived retinal cultures following the expression of two microRNAs, miR-182 and miR183 (Busskamp et al., 2014).

In the study by Gonzalez-Cordero et al., ESC-derived photoreceptor precursors were virally labelled with AAV2/9. Rhodopsin.GFP and purified by FACS. Sorted rods (200,000 cells per eye) were then transplanted to the subretinal space of the adult Gnat $1^{-/}$mouse model of degeneration (Figure 4). Using this method, ESC-derived photoreceptors were reported to (i) migrate and integrate into the appropriate location in the ONL, (ii) display morphology of mature rod photoreceptors, (iii) form synapse-like contacts with bipolar cells within the INL and (iv) contain the phototransduction protein rod a-transducin, which is absent in the photoreceptors of the host retina. It was also demonstrated that ESC-derived photoreceptor precursors could integrate in the Prph $2^{r d 2 / r d 2}$ and, to a certain extent, the $R h o^{-/}$models of degeneration in a manner similar to other studies (Barber et al., 2013). Importantly, cells isolated from earlier cultures (days 26-29), comparable to early postnatal stages in the mouse and hence representing a more immature rod photoreceptor phenotype, integrated in higher numbers than those isolated from late cultures (day 34). In this regard, the ESCderived photoreceptor precursors behaved very similarly to the freshly dissociated Nrl.GFPpositive photoreceptor precursors from postnatal retinas (MacLaren et al., 2006; Pearson et al., 2012). However, despite these similarities, it is important to emphasise that an average of just 500 integrated photoreceptors were observed three weeks post-transplantation in the Gnat ${ }^{-1}$ recipient, compared to an average of 5,000-10,000 cells following single injections of donor photoreceptors from P4-8 mice retinas. Given these low numbers of integrated cells, no visual rescue assessments were attempted in this study.

In close agreement with Gonzalez-Cordero et al., Arsenijivic and colleagues, reported findings using a mouse ESC-line obtained from the Crx.GFP mouse. They reported that, following transplantation, developmentally stage-specific photoreceptors obtained from the 3D culture system migrate and integrate within the recipient retina (Decembrini et al., 2014). These Crx.GFP-positive cells were transplanted subretinally into the immunodeficient non- 
obese diabetic/severe combined immunodeficient (NOD/SCID) adult retina. Photoreceptors derived from day 25 cultures integrated within the recipient ONL in higher numbers than donors taken from day 30 cultures. Decembrini et al., reported numbers of integrated cells very similar to those reported by Gonzalez-Cordero et al. despite the use of immune deficient animals, suggesting that these non-autologous cells are not subject to immune rejection, at least at one month post-injection (Decembrini et al., 2014).

Another approach for cell replacement strategies involves the transplantation of retinal sheets, rather than dissociated cells. This strategy could be of considerable interest, particularly for the treatment of very severe degenerations where the entire ONL is absent. It has a distinct advantage over cell transplantation in the potential extent of photoreceptor replacement. A number of groups have examined the potential for transplanting retinal progenitor and full thickness retinal sheets, some including RPE. Collectively, these studies show graft survival, connectivity and function in animal models [reviewed by (Seiler and Aramant, 2012)] and patients receiving retinal progenitor sheet transplants demonstrated improvements in vision (Radtke et al., 2008). As yet, it is unclear to what extent normal visual processing will be affected by the inclusion of inner retinal neurons within the retinal sheet. Silverman and colleagues pioneered techniques to derive photoreceptor sheets from adult and early postnatal retina (Silverman et al., 1992; Silverman and Hughes, 1989a; Silverman and Hughes, 1989b; Silverman and Hughes, 1990). Ghosh and colleagues (Ghosh et al., 1999) reported limited success following the transplantation of partialthickness sheets into the rabbit, most probably due to damage incurred during tissue processing. Unfortunately such isolation techniques necessarily induce trauma to the tissue. A second, more practical issue is the surgical logistics of getting a fragile tissue sheet into the recipient subretinal space and ensuring that it lies flat against the recipient neural retinal surface. Supply of fetal donor tissue is, inevitably, limited. Interestingly, however, Takahashi and colleagues adapted the Eiraku/Sasai 3D-differentiation protocol to differentiate mouse ES or iPS cells into neuroepithelium structures containing a defined ONL (Assawachananont et al., 2014). Subsequently, these mES/iPSC-derived retinal sheets, obtained from differently-staged cultures [young (day 11-17) and old (day 18-24)], were transplanted into the $R d 1$ mouse, an animal model for rapid and severe degeneration in which the ONL is almost completely lost by three weeks (Lolley et al., 1994). Following transplantation, photoreceptor precursors in the transplanted ONL were shown to form inner and outer segments, as demonstrated by the presence of Recoverin and Rhodopsin. Furthermore, the authors evaluated the integration potential of these sheets with the host retina by analysing the connection with the host bipolar cells via immunostaining for PKC. They observed two possible outcomes; in some instances, the transplanted stem cell-derived neuroepithelium 
contained an INL and, therefore, interneurons were present between host INL and transplanted ONL. However, when the transplanted sheets did not contain a proper INL, the ESC-derived ONL readily contacted the host INL. Under these circumstances, the donor photoreceptors make physical contacts with the host bipolar cells. Further studies are required to determine whether these represent functional synaptic connections and whether these ESC-derived photoreceptor sheets are able to drive visual function. Another important consideration is the contact between the transplanted photoreceptors' newly formed outer segments and the endogenous RPE. Again, while the outer segments from the stem cellderived photoreceptors were sometimes well aligned and in contact with the host RPE, other regions formed rosettes, which inhibited direct contact with the RPE. In summary, the last year has seen the publication of a number of reports from different groups demonstrating the competence of mouse ESC and iPSC-derived photoreceptors to survive and make contact with the recipient retina following transplantation (Assawachananont et al., 2014; Decembrini et al., 2014; Gonzalez-Cordero et al., 2013; Tucker et al., 2013). Improvements in transplantation efficiency represent the next essential step; this includes not only improving the number of cells that integrate within the diseased recipient retina, but also ensuring their continued survival. We must also determine whether these cells truly integrate into the recipient retinal circuitry and are capable of driving visual function. Most importantly, the recently described human ESC/iPSC-derived photoreceptors (Nakano et al., 2012; Zhong et al., 2014) must now be assessed for their capacity to migrate and integrate into the recipient retina and whether they can also drive visual function. To date, only one study has reported the transplantation of human ESC-derived photoreceptors (Lamba et al., 2009) and therefore more detailed transplantation assessments are required to identify the optimal stage of maturation for robust integration.

\section{Clinical Perspectives}

The prospects for taking photoreceptor replacement therapy towards clinical application look promising. The past decade has seen major advances, including the demonstration of visual function following photoreceptor transplantation and the generation of transplantable donor cells from renewable sources. We have also seen that it is possible to transplant cells into even very severely diseased retinas. Nonetheless, there are many significant challenges to overcome before taking this into the clinic. The efficiency of donor cell integration after transplantation into the diseased retina must be significantly improved, particularly using ESC/iPSC-derived sources. Achieving this will undoubtedly require the full elucidation of the intrinsic and extrinsic factors regulating photoreceptor development and maturation. Not all degenerations are the same and each presents its own set of barriers that may impede the 
passage of donor cells from the site of transplantation into the recipient retina (see (Barber et al., 2013)). The remaining retinal circuitry will also undergo changes that may affect the processing of new visual information in an as yet unknown manner. Once there, the transplanted cells may, depending upon their source and the level of inflammation in the recipient eye, face challenges from the host immune system.

The development of human ESC/iPSC-derived photoreceptor differentiation methods suitable for clinical use will be an essential phase of preparation towards future clinical studies involving photoreceptor transplantation. Points to consider include the choice of ES or iPS cell lines and differentiation protocol to be used, development of storage strategies and donor cell isolation and purification methods. Importantly, all of these criteria will need to be delivered under good manufacturing practice (GMP) compliant conditions.

While ESCs have a great capacity for expansion and differentiation and, therefore, represent a suitable source of cells for transplantation therapies, there are some concerns related to immunogenicity. The transplanted population could trigger a host immune rejection and, thus, treatments could be compromised (see (Pearson et al., 2014)). Our understanding of the host immunological responses following photoreceptor transplantation is still very limited, having only been assessed in a handful of studies. A detailed characterisation of the innate and adaptive responses following transplantation is required if ESC-derived photoreceptors are to be used in clinical settings. The use of donor cells generated from iPS cell lines that are human leukocyte antigen (HLA)-haplotype matched may avoid this issue and there are many initiatives around the world to set up human iPS cell banks, with such aims in mind.

To take any differentiation protocol forward to clinical application, it must be GMP compliant and use clinical grade lines to generate, store and purify human ESC/iPSC-derived photoreceptors. The majority of the differentiation protocols discussed in section 8.1 involve animal-derived products. Xeno-contaminated cells are unsuitable for transplantation into patients because of the risk of zoonoses and the potential activation of animal retroviruses, as well as the possibility of immune rejection. Although some xeno-free differentiation protocols to generate retinal cell types have been investigated (Sridhar et al., 2013; Tucker et al., 2013), there is still much optimisation required before we can generate transplantation-competent photoreceptor precursors (or indeed retinal sheets) under GMPcompliant conditions. The combination of knowledge obtained from donor-derived, mouse and human ESC-derived photoreceptor precursor transplantation studies will assist the development of a defined scalable, non-xenogeneic protocol for generating, purifying and storing hESC-derived photoreceptor precursors. 
The differentiation of human ESC/iPSC-derived photoreceptors requires long-term cultures (Nakano et al., 2012; Zhong et al., 2014). This lengthy differentiation is not unexpected if compared to human eye development. However, keeping these cultures is not only laborious and costly for the researcher but also makes it harder to translate to large-scale preparations, required for clinical purposes. Therefore, the development of appropriate cryopreservation methods suitable for photoreceptor precursors will be important so that cells at the right stage of development can be obtained at any time. It is also extremely important to develop a selection strategy for hESC/iPSC-derived photoreceptors that will separate these cells from the inherently heterogeneous mix of cells derived from pluripotent stem cell cultures. We, and others, previously described the transplantation of mouse photoreceptor precursors via selection employing cell surface antigens, CD markers (Eberle et al., 2011; Lakowski et al., 2010; Lakowski et al., 2011). Rod photoreceptor precursor cells isolated on the basis of $\mathrm{CD} 73^{+} / \mathrm{CD} 24^{+}$expression integrate into the adult wild type mouse retina in numbers similar, or even higher, than cells selected on the basis of Nrl.GFP (Lakowski et al., 2011). We are now characterising the presence of these, and other, cell surface markers in mouse ESC-derived photoreceptors to establish their potential as markers to isolate these cells for transplantation (Lakowski et al., manuscript in revision). We are also assessing if the same and/or other cell surface markers are present in stage specific human photoreceptor cells.

Importantly, much of the work reported to date has focused on rod transplantation in animal models, typically rodents. While it is possible that rod transplantation may benefit patients with significant rod loss, the challenges to widespread replacement are significant, as mentioned above. A more realistic aim, perhaps, is local replacement, particularly of cone photoreceptors. Transplantation of cone or cone-like photoreceptors has been investigated in a small number of murine models, but the numbers of integrated cones is markedly lower than that of rods (Lakowski et al., 2010; Santos-Ferreira et al., 2014). It will be essential to define strategies for the generation of transplantable ESC/iPSC-derived cones. We also need to understand the mechanisms governing the integration, maturation and survival of these cells following transplantation into the diseased retina. Synaptic connectivity must also be considered since correct visual processing relies on the establishment of numerous synaptic connections between cones and the inner retina, compared to the almost one-toone synaptic connectivity of rods and rod bipolar cells. Cone transplantation raises the question of whether rodents, as nocturnal, rod-dominant animals, represent the best model on which to test the integration of human photoreceptors. In the absence of alternatives, it is logical to continue with these experimental models to identify limitations, particularly within 
the recipient retina, and to define strategies to overcome them. Nonetheless, we must be prepared for many unforeseen challenges when moving to the human retina.

Finally, it is important to remember that cell therapy is just one of a number of novel therapeutic strategies under investigation. At this point in time, it is very hard to make predictions as to which will be the 'right' strategy. More likely, different types of disease will most likely be receptive to different strategies, even within a single category, such as RP, depending on the precise nature of the resulting degeneration.

\section{Conclusions}

Built on a background of more than 50 years of research in retinal development and transplantation strategies, the last decade has seen enormous progress in both photoreceptor replacement and regeneration strategies. The study of lower vertebrates has enabled us to identify populations of cells with stem cell-like potential in ocular structures as diverse as the post-mitotic neural retina and the CB. Although it has yet to be shown conclusively that these sources are capable of generating transplantation-competent cells for photoreceptor transplantation, they do raise the prospect of reactivating endogenous repair mechanisms. This has only been possible through our understanding of these mechanisms in lower vertebrates, which themselves are very often the reactivation of processes occurring during embryogenesis. Restoration of visual function by cell replacement strategies is a real possibility. This too has been underpinned by knowledge of photoreceptor neurogenesis, permitting the identification of markers that identify transplantation-competent cells. Finally, we are now able to direct the generation of structures as complex as the mammalian retina from ES and iPS cells in vitro by presenting exogenous stimuli in a pattern that recapitulates normal retinal development in a dish. In a time of immense translational progress, it is essential that we do not forget that these advances are, almost without exception, built on the foundations of basic research into retinal development. 


\section{Reference List}

Aasen,T., Raya,A., Barrero,M.J., Garreta,E., Consiglio,A., Gonzalez,F., Vassena,R., Bilic,J., Pekarik,V., Tiscornia,G. et al. (2008). Efficient and rapid generation of induced pluripotent stem cells from human keratinocytes. Nat. Biotechnol. 26, 1276-1284.

Abdouh,M. and Bernier,G. (2006). In vivo reactivation of a quiescent cell population located in the ocular ciliary body of adult mammals. Exp. Eye Res. 83, 153-164.

Ahmad,I., Tang,L., and Pham,H. (2000). Identification of neural progenitors in the adult mammalian eye. Biochem. Biophys. Res. Commun. 270, 517-521.

Akagi,T., Akita,J., Haruta,M., Suzuki,T., Honda,Y., Inoue,T., Yoshiura,S., Kageyama,R., Yatsu,T., Yamada,M. et al. (2005). Iris-derived cells from adult rodents and primates adopt photoreceptor-specific phenotypes. Invest Ophthalmol. Vis. Sci. 46, 3411-3419.

Akagi,T., Mandai,M., Ooto,S., Hirami,Y., Osakada,F., Kageyama,R., Yoshimura,N., and Takahashi,M. (2004). Otx2 homeobox gene induces photoreceptor-specific phenotypes in cells derived from adult iris and ciliary tissue. Invest Ophthalmol. Vis. Sci. 45, 4570-4575.

Akimoto,M., Cheng,H., Zhu,D., Brzezinski,J.A., Khanna,R., Filippova,E., Oh,E.C., Jing,Y., Linares,J.L., Brooks,M. et al. (2006). Targeting of GFP to newborn rods by Nrl promoter and temporal expression profiling of flow-sorted photoreceptors. Proc. Natl. Acad. Sci. U. S. A 103, 3890-3895.

Al-Hussaini,H., Kam,J.H., Vugler,A., Semo,M., and Jeffery,G. (2008). Mature retinal pigment epithelium cells are retained in the cell cycle and proliferate in vivo. Mol. Vis. 14, 1784-1791.

Ali,R.R. (2012). Ocular gene therapy: introduction to the special issue. Gene Ther. 19, 119120.

Amato,M.A., Arnault,E., and Perron,M. (2004). Retinal stem cells in vertebrates: parallels and divergences. Int. J. Dev. Biol. 48, 993-1001.

Anchan,R.M., Reh,T.A., Angello,J., Balliet,A., and Walker,M. (1991). EGF and TGF-alpha stimulate retinal neuroepithelial cell proliferation in vitro. Neuron 6, 923-936.

Andreazzoli,M. (2009). Molecular regulation of vertebrate retina cell fate. Birth Defects Res.

C. Embryo. Today 87, 284-295.

Arnhold,S., Semkova,I., Andressen,C., Lenartz,D., Meissner,G., Sturm,V., Kochanek,S., Addicks,K., and Schraermeyer,U. (2004). Iris pigment epithelial cells: a possible cell source for the future treatment of neurodegenerative diseases. Exp. Neurol. 187, 410-417.

Asami,M., Sun,G., Yamaguchi,M., and Kosaka,M. (2007). Multipotent cells from mammalian iris pigment epithelium. Dev. Biol. 304, 433-446.

Assawachananont,J., Mandai,M., Okamoto,S., Yamada,C., Eiraku,M., Yonemura,S., Sasai,Y., and Takahashi,M. (2014). Transplantation of Embryonic and Induced Pluripotent 
Stem Cell-Derived 3D Retinal Sheets into Retinal Degenerative Mice. Stem Cell Reports. 2, 662-674.

Bain,G., Kitchens,D., Yao,M., Huettner,J.E., and Gottlieb,D.I. (1995). Embryonic stem cells express neuronal properties in vitro. Dev. Biol. 168, 342-357.

Bainbridge,J.W., Smith,A.J., Barker,S.S., Robbie,S., Henderson,R., Balaggan,K., Viswanathan,A., Holder,G.E., Stockman,A., Tyler,N. et al. (2008). Effect of gene therapy on visual function in Leber's congenital amaurosis. N. Engl. J. Med. 358, 2231-2239.

Balenci,L., Wonders,C., Coles,B.L., Clarke,L., and Van Der Kooy,D. (2013). Bone morphogenetic proteins and secreted frizzled related protein 2 maintain the quiescence of adult mammalian retinal stem cells. Stem Cells 31, 2218-2230.

Ballios,B.G., Clarke,L., Coles,B.L., Shoichet,M.S., and Van Der Kooy,D. (2012). The adult retinal stem cell is a rare cell in the ciliary epithelium whose progeny can differentiate into photoreceptors. Biol. Open. 1, 237-246.

Banin,E., Obolensky,A., Idelson,M., Hemo,I., Reinhardtz,E., Pikarsky,E., Ben-Hur,T., and Reubinoff,B. (2006). Retinal incorporation and differentiation of neural precursors derived from human embryonic stem cells. Stem Cells 24, 246-257.

Barber,A.C., Hippert,C., Duran,Y., West,E.L., Bainbridge,J.W., Warre-Cornish,K., Luhmann,U.F., Lakowski,J., Sowden,J.C., Ali,R.R. et al. (2013). Repair of the degenerate retina by photoreceptor transplantation. Proc. Natl. Acad. Sci. U. S. A 110, 354-359.

Barbosa-Sabanero,K., Hoffmann,A., Judge,C., Lightcap,N., Tsonis,P.A., and Del RioTsonis,K. (2012). Lens and retina regeneration: new perspectives from model organisms. Biochem. J. 447, 321-334.

Bartsch,U., Oriyakhel,W., Kenna,P.F., Linke,S., Richard,G., Petrowitz,B., Humphries,P., Farrar,G.J., and Ader,M. (2008). Retinal cells integrate into the outer nuclear layer and differentiate into mature photoreceptors after subretinal transplantation into adult mice. Exp. Eye Res. 86, 691-700.

Bernardos,R.L., Barthel,L.K., Meyers,J.R., and Raymond,P.A. (2007). Late-stage neuronal progenitors in the retina are radial Muller glia that function as retinal stem cells. $J$. Neurosci. 27, 7028-7040.

Bhatia,B., Singhal,S., Lawrence,J.M., Khaw,P.T., and Limb,G.A. (2009). Distribution of Muller stem cells within the neural retina: evidence for the existence of a ciliary margin-like zone in the adult human eye. Exp. Eye Res. 89, 373-382.

Blackshaw,S., Harpavat,S., Trimarchi,J., Cai,L., Huang,H., Kuo,W.P., Weber,G., Lee,K., Fraioli,R.E., Cho,S.H. et al. (2004). Genomic analysis of mouse retinal development. PLoS. Biol. 2, E247. 
Bone-Larson,C., Basu,S., Radel,J.D., Liang,M., Perozek,T., Kapousta-Bruneau,N., Green,D.G., Burmeister,M., and Hankin,M.H. (2000). Partial rescue of the ocular retardation phenotype by genetic modifiers. J. Neurobiol. 42, 232-247.

Borday,C., Cabochette,P., Parain,K., Mazurier,N., Janssens,S., Tran,H.T., Sekkali,B., Bronchain,O., Vleminckx,K., Locker,M. et al. (2012). Antagonistic cross-regulation between Wnt and Hedgehog signalling pathways controls post-embryonic retinal proliferation. Development 139, 3499-3509.

Borooah,S., Phillips,M.J., Bilican,B., Wright,A.F., Wilmut,I., Chandran,S., Gamm,D., and Dhillon,B. (2013). Using human induced pluripotent stem cells to treat retinal disease. Prog. Retin. Eye Res. 37, 163-181.

Boucherie,C., Mukherjee,S., Henckaerts,E., Thrasher,A.J., Sowden,J.C., and Ali,R.R. (2013). Brief report: self-organizing neuroepithelium from human pluripotent stem cells facilitates derivation of photoreceptors. Stem Cells 31, 408-414.

Bouwmeester,T., Kim,S., Sasai,Y., Lu,B., and De Robertis,E.M. (1996). Cerberus is a head-inducing secreted factor expressed in the anterior endoderm of Spemann's organizer. Nature 382, 595-601.

Bradbury,M.S., Panagiotakos,G., Chan,B.K., Tomishima,M., Zanzonico,P., Vider,J., Ponomarev,V., Studer,L., and Tabar,V. (2007). Optical bioluminescence imaging of human ES cell progeny in the rodent CNS. J. Neurochem. 102, 2029-2039.

Bringmann,A., Pannicke,T., Grosche,J., Francke,M., Wiedemann,P., Skatchkov,S.N., Osborne,N.N., and Reichenbach,A. (2006). Muller cells in the healthy and diseased retina. Prog. Retin. Eye Res. 25, 397-424.

Brzezinski,J.A., Lamba,D.A., and Reh,T.A. (2010). Blimp1 controls photoreceptor versus bipolar cell fate choice during retinal development. Development 137, 619-629.

Buch,P.K., MacLaren,R.E., and Ali,R.R. (2007). Neuroprotective gene therapy for the treatment of inherited retinal degeneration. Curr. Gene Ther. 7, 434-445.

Bunce,C., Xing,W., and Wormald,R. (2010). Causes of blind and partial sight certifications in England and Wales: April 2007-March 2008. Eye (Lond) 24, 1692-1699.

Bunker,C.H., Berson,E.L., Bromley,W.C., Hayes,R.P., and Roderick,T.H. (1984). Prevalence of retinitis pigmentosa in Maine. Am. J. Ophthalmol. 97, 357-365.

Burmeister,M., Novak,J., Liang,M.Y., Basu,S., Ploder,L., Hawes,N.L., Vidgen,D., Hoover,F., Goldman,D., Kalnins,V.I. et al. (1996). Ocular retardation mouse caused by Chx10 homeobox null allele: impaired retinal progenitor proliferation and bipolar cell differentiation. Nat. Genet. 12, 376-384.

Busskamp,V., Krol,J., Nelidova,D., Daum,J., Szikra,T., Tsuda,B., Juttner,J., Farrow,K., Scherf,B.G., Alvarez,C.P. et al. (2014). miRNAs 182 and 183 Are Necessary to Maintain Adult Cone Photoreceptor Outer Segments and Visual Function. Neuron. 
Busskamp,V., Picaud,S., Sahel,J.A., and Roska,B. (2012). Optogenetic therapy for retinitis pigmentosa. Gene Ther. 19, 169-175.

Campochiaro,P.A. (2006). Ocular versus extraocular neovascularization: mirror images or vague resemblances. Invest Ophthalmol. Vis. Sci. 47, 462-474.

Carl,M. and Wittbrodt,J. (1999). Graded interference with FGF signalling reveals its dorsoventral asymmetry at the mid-hindbrain boundary. Development 126, 5659-5667.

Carter-Dawson,L.D. and LaVail,M.M. (1979a). Rods and cones in the mouse retina. I. Structural analysis using light and electron microscopy. J. Comp Neurol. 188, 245-262.

Carter-Dawson,L.D. and LaVail,M.M. (1979b). Rods and cones in the mouse retina. II. Autoradiographic analysis of cell generation using tritiated thymidine. J. Comp Neurol. 188, 263-272.

Caspi,A., Dorn,J.D., McClure,K.H., Humayun,M.S., Greenberg,R.J., and McMahon,M.J. (2009). Feasibility study of a retinal prosthesis: spatial vision with a 16-electrode implant. Arch. Ophthalmol. 127, 398-401.

Centanin,L., Hoeckendorf,B., and Wittbrodt,J. (2011). Fate restriction and multipotency in retinal stem cells. Cell Stem Cell 9, 553-562.

Cepko,C. (2014). Intrinsically different retinal progenitor cells produce specific types of progeny. Nat. Rev. Neurosci. 15, 615-627.

Cepko,C.L. (1999). The roles of intrinsic and extrinsic cues and bHLH genes in the determination of retinal cell fates. Curr. Opin. Neurobiol. 9, 37-46.

Cepko,C.L., Austin,C.P., Yang,X., Alexiades,M., and Ezzeddine,D. (1996). Cell fate determination in the vertebrate retina. Proc. Natl. Acad. Sci. U. S. A 93, 589-595.

Chang,C. and Hemmati-Brivanlou,A. (1998). Neural crest induction by Xwnt7B in Xenopus. Dev. Biol. 194, 129-134.

Chen,J., Rattner,A., and Nathans,J. (2005). The rod photoreceptor-specific nuclear receptor Nr2e3 represses transcription of multiple cone-specific genes. J. Neurosci. 25, 118129.

Chen,S., Li,H., Gaudenz,K., Paulson,A., Guo,F., Trimble,R., Peak,A., Seidel,C., Deng,C., Furuta,Y. et al. (2013). Defective FGF signaling causes coloboma formation and disrupts retinal neurogenesis. Cell Res. 23, 254-273.

Chow,R.L. and Lang,R.A. (2001). Early eye development in vertebrates. Annu. Rev. Cell Dev. Biol. 17, 255-296.

Cicero,S.A., Johnson,D., Reyntjens,S., Frase,S., Connell,S., Chow,L.M., Baker,S.J., Sorrentino,B.P., and Dyer,M.A. (2009). Cells previously identified as retinal stem cells are pigmented ciliary epithelial cells. Proc. Natl. Acad. Sci. U. S. A 106, 6685-6690. 
Close,J.L., Gumuscu,B., and Reh,T.A. (2005). Retinal neurons regulate proliferation of postnatal progenitors and Muller glia in the rat retina via TGF beta signaling. Development 132, 3015-3026.

Close,J.L., Liu,J., Gumuscu,B., and Reh,T.A. (2006). Epidermal growth factor receptor expression regulates proliferation in the postnatal rat retina. Glia 54, 94-104.

Coles,B.L., Angenieux,B., Inoue,T., Del Rio-Tsonis,K., Spence,J.R., McInnes,R.R., Arsenijevic,Y., and Van Der Kooy,D. (2004). Facile isolation and the characterization of human retinal stem cells. Proc. Natl. Acad. Sci. U. S. A 101, 15772-15777.

Crespo,D., Asher,R.A., Lin,R., Rhodes,K.E., and Fawcett,J.W. (2007). How does chondroitinase promote functional recovery in the damaged CNS? Exp. Neurol. 206, 159171.

Damiani,D., Novelli,E., Mazzoni,F., and Strettoi,E. (2012). Undersized dendritic arborizations in retinal ganglion cells of the rd1 mutant mouse: a paradigm of early onset photoreceptor degeneration. J. Comp Neurol. 520, 1406-1423.

Das,A.V., James,J., Rahnenfuhrer,J., Thoreson,W.B., Bhattacharya,S., Zhao,X., and Ahmad,I. (2005). Retinal properties and potential of the adult mammalian ciliary epithelium stem cells. Vision Res. 45, 1653-1666.

Das,A.V., James,J., Zhao,X., Rahnenfuhrer,J., and Ahmad,I. (2004). Identification of c-Kit receptor as a regulator of adult neural stem cells in the mammalian eye: interactions with Notch signaling. Dev. Biol. 273, 87-105.

Das,A.V., Mallya,K.B., Zhao,X., Ahmad,F., Bhattacharya,S., Thoreson,W.B., Hegde,G.V., and Ahmad,I. (2006a). Neural stem cell properties of Muller glia in the mammalian retina: regulation by Notch and Wnt signaling. Dev. Biol. 299, 283-302.

Das,A.V., Zhao,X., James,J., Kim,M., Cowan,K.H., and Ahmad,I. (2006b). Neural stem cells in the adult ciliary epithelium express GFAP and are regulated by Wnt signaling. Biochem. Biophys. Res. Commun. 339, 708-716.

Davidson,B.P., Cheng,L., Kinder,S.J., and Tam,P.P. (2000). Exogenous FGF-4 can suppress anterior development in the mouse embryo during neurulation and early organogenesis. Dev. Biol. 221, 41-52.

Davis,A.A., Matzuk,M.M., and Reh,T.A. (2000). Activin A promotes progenitor differentiation into photoreceptors in rodent retina. Mol. Cell Neurosci. 15, 11-21.

Davis,N., Yoffe,C., Raviv,S., Antes,R., Berger,J., Holzmann,S., Stoykova,A., Overbeek,P.A., Tamm,E.R., and Ashery-Padan,R. (2009). Pax6 dosage requirements in iris and ciliary body differentiation. Dev. Biol. 333, 132-142.

De Jong,P.T. (2006). Age-related macular degeneration. N. Engl. J. Med. 355, 1474-1485. 
Decembrini,S., Koch,U., Radtke,F., Moulin,A., and Arsenijevic,Y. (2014). Derivation of traceable and transplantable photoreceptors from mouse embryonic stem cells. Stem Cell Reports. 2, 853-865.

Del Debbio,C.B., Balasubramanian,S., Parameswaran,S., Chaudhuri,A., Qiu,F., and Ahmad,I. (2010). Notch and Wnt signaling mediated rod photoreceptor regeneration by Muller cells in adult mammalian retina. PLoS. One. 5, e12425.

Del Debbio,C.B., Peng,X., Xiong,H., and Ahmad,I. (2013). Adult ciliary epithelial stem cells generate functional neurons and differentiate into both early and late born retinal neurons under non-cell autonomous influences. BMC. Neurosci. 14, 130.

Del Debbio,C.B., Santos,M.F., Yan,C.Y., Ahmad,I., and Hamassaki,D.E. (2014). Rho GTPases control ciliary epithelium cells proliferation and progenitor profile induction in vivo. Invest Ophthalmol. Vis. Sci. 55, 2631-2641.

Demontis,G.C., Aruta,C., Comitato,A., De,M.A., and Marigo,V. (2012). Functional and molecular characterization of rod-like cells from retinal stem cells derived from the adult ciliary epithelium. PLoS. One. 7, e33338.

Denayer,T., Locker,M., Borday,C., Deroo,T., Janssens,S., Hecht,A., van,R.F., Perron,M., and Vleminckx,K. (2008a). Canonical Wnt signaling controls proliferation of retinal stem/progenitor cells in postembryonic Xenopus eyes. Stem Cells 26, 2063-2074.

Denayer,T., Tran,H.T., and Vleminckx,K. (2008b). Transgenic reporter tools tracing endogenous canonical Wnt signaling in Xenopus. Methods Mol. Biol. 469, 381-400.

Dowling.J.E. (2012). The Retina: An Approachable Part of the Brain. Harvard University Press.

Durston,A.J., Timmermans,J.P., Hage,W.J., Hendriks,H.F., de Vries,N.J., Heideveld,M., and Nieuwkoop,P.D. (1989). Retinoic acid causes an anteroposterior transformation in the developing central nervous system. Nature 340, 140-144.

Dyer,M.A. and Cepko,C.L. (2000). Control of Muller glial cell proliferation and activation following retinal injury. Nat. Neurosci. 3, 873-880.

Eberle,D., Kurth,T., Santos-Ferreira,T., Wilson,J., Corbeil,D., and Ader,M. (2012). Outer segment formation of transplanted photoreceptor precursor cells. PLoS. One. 7, e46305.

Eberle,D., Schubert,S., Postel,K., Corbeil,D., and Ader,M. (2011). Increased integration of transplanted CD73-positive photoreceptor precursors into adult mouse retina. Invest Ophthalmol. Vis. Sci. 52, 6462-6471.

Eiraku,M., Takata,N., Ishibashi,H., Kawada,M., Sakakura,E., Okuda,S., Sekiguchi,K., Adachi,T., and Sasai,Y. (2011). Self-organizing optic-cup morphogenesis in threedimensional culture. Nature 472, 51-56. 
EI,Y.W., Borday,C., Hamdache,J., Parain,K., Tran,H.T., Vleminckx,K., Perron,M., and Locker,M. (2012). Hes4 controls proliferative properties of neural stem cells during retinal ontogenesis. Stem Cells 30, 2784-2795.

Eminli,S., Foudi,A., Stadtfeld,M., Maherali,N., Ahfeldt,T., Mostoslavsky,G., Hock,H., and Hochedlinger,K. (2009). Differentiation stage determines potential of hematopoietic cells for reprogramming into induced pluripotent stem cells. Nat. Genet. 41, 968-976.

Engelhardt,M., Bogdahn,U., and Aigner,L. (2005). Adult retinal pigment epithelium cells express neural progenitor properties and the neuronal precursor protein doublecortin. Brain Res. 1040, 98-111.

Engelhardt,M., Wachs,F.P., Couillard-Despres,S., and Aigner,L. (2004). The neurogenic competence of progenitors from the postnatal rat retina in vitro. Exp. Eye Res. 78, 10251036.

England,S.J., Blanchard,G.B., Mahadevan,L., and Adams,R.J. (2006). A dynamic fate map of the forebrain shows how vertebrate eyes form and explains two causes of cyclopia. Development 133, 4613-4617.

Evans,J.R. (2001). Risk factors for age-related macular degeneration. Prog. Retin. Eye Res. 20, 227-253.

Evans,M.J. and Kaufman,M.H. (1981). Establishment in culture of pluripotential cells from mouse embryos. Nature 292, 154-156.

Fausett,B.V. and Goldman,D. (2006). A role for alpha1 tubulin-expressing Muller glia in regeneration of the injured zebrafish retina. J. Neurosci. 26, 6303-6313.

Fawcett,J. (2009). Molecular control of brain plasticity and repair. Prog. Brain Res. 175, 501-509.

Finger,R.P., Wickremasinghe,S.S., Baird,P.N., and Guymer,R.H. (2014). Predictors of anti-VEGF treatment response in neovascular age-related macular degeneration. Surv. Ophthalmol. 59, 1-18.

Fischer,A.J. and Reh,T.A. (2000). Identification of a proliferating marginal zone of retinal progenitors in postnatal chickens. Dev. Biol. 220, 197-210.

Fischer,A.J. and Reh,T.A. (2003). Potential of Muller glia to become neurogenic retinal progenitor cells. Glia 43, 70-76.

Freddo,T.F. (1996). Ultrastructure of the iris. Microsc. Res. Tech. 33, 369-389.

Freund,C.L., Gregory-Evans,C.Y., Furukawa,T., Papaioannou,M., Looser,J., Ploder,L., Bellingham,J., Ng,D., Herbrick,J.A., Duncan,A. et al. (1997). Cone-rod dystrophy due to mutations in a novel photoreceptor-specific homeobox gene (CRX) essential for maintenance of the photoreceptor. Cell 91, 543-553.

Freund,C.L., Wang,Q.L., $\quad$ Chen,S., $\quad$ Muskat,B.L., $\quad$ Wiles,C.D., $\quad$ Sheffield,V.C., Jacobson,S.G., Mclnnes,R.R., Zack,D.J., and Stone,E.M. (1998). De novo mutations in 
the CRX homeobox gene associated with Leber congenital amaurosis. Nat. Genet. 18, 311312.

Friedlander,D.R., Milev,P., Karthikeyan,L., Margolis,R.K., Margolis,R.U., and Grumet,M. (1994). The neuronal chondroitin sulfate proteoglycan neurocan binds to the neural cell adhesion molecules $\mathrm{Ng}$-CAM/L1/NILE and N-CAM, and inhibits neuronal adhesion and neurite outgrowth. J. Cell Biol. 125, 669-680.

Froen,R.C., Johnsen,E.O., Petrovski,G., Berenyi,E., Facsko,A., Berta,A., Nicolaissen,B., and Moe,M.C. (2011). Pigment epithelial cells isolated from human peripheral iridectomies have limited properties of retinal stem cells. Acta Ophthalmol. 89, e635-e644.

Fuhrmann,S. (2010). Eye morphogenesis and patterning of the optic vesicle. Curr. Top. Dev. Biol. 93, 61-84.

Furukawa,T., Morrow,E.M., Li,T., Davis,F.C., and Cepko,C.L. (1999). Retinopathy and attenuated circadian entrainment in Crx-deficient mice. Nat. Genet. 23, 466-470.

Gai,H., Leung,E.L., Costantino,P.D., Aguila,J.R., Nguyen,D.M., Fink,L.M., Ward,D.C., and Ma,Y. (2009). Generation and characterization of functional cardiomyocytes using induced pluripotent stem cells derived from human fibroblasts. Cell Biol. Int. 33, 1184-1193.

Gallina,D., Todd,L., and Fischer,A.J. (2013). A comparative analysis of Muller gliamediated regeneration in the vertebrate retina. Exp. Eye Res.

Gamm,D.M., Wang,S., Lu,B., Girman,S., Holmes,T., Bischoff,N., Shearer,R.L., Sauve,Y., Capowski,E., Svendsen,C.N. et al. (2007). Protection of visual functions by human neural progenitors in a rat model of retinal disease. PLoS. One. 2, e338.

Gamse,J. and Sive,H. (2000). Vertebrate anteroposterior patterning: the Xenopus neurectoderm as a paradigm. Bioessays 22, 976-986.

Ghosh,F. and Ehinger,B. (2000). Full-thickness retinal transplants: a review. Ophthalmologica 214, 54-69.

Ghosh,F., Juliusson,B., Arner,K., and Ehinger,B. (1999). Partial and full-thickness neuroretinal transplants. Exp. Eye Res. 68, 67-74.

Gilbert,R.J., McKeon,R.J., Darr,A., Calabro,A., Hascall,V.C., and Bellamkonda,R.V. (2005). CS-4,6 is differentially upregulated in glial scar and is a potent inhibitor of neurite extension. Mol. Cell Neurosci. 29, 545-558.

Giordano,F., De,M.A., Vetrini,F., and Marigo,V. (2007). Fibroblast growth factor and epidermal growth factor differently affect differentiation of murine retinal stem cells in vitro. Mol. Vis. 13, 1842-1850.

Giorgetti,A., Montserrat,N., Aasen,T., Gonzalez,F., Rodriguez-Piza,I., Vassena,R., Raya,A., Boue,S., Barrero,M.J., Corbella,B.A. et al. (2009). Generation of induced 
pluripotent stem cells from human cord blood using OCT4 and SOX2. Cell Stem Cell 5, 353357.

Glinka,A., Wu,W., Delius,H., Monaghan,A.P., Blumenstock,C., and Niehrs,C. (1998). Dickkopf-1 is a member of a new family of secreted proteins and functions in head induction. Nature 391, 357-362.

Goldman,D. (2014). Muller glial cell reprogramming and retina regeneration. Nat. Rev. Neurosci. 15, 431-442.

Gonzalez-Cordero,A., West,E.L., Pearson,R.A., Duran,Y., Carvalho,L.S., Chu,C.J., Naeem,A., Blackford,S.J., Georgiadis,A., Lakowski,J. et al. (2013). Photoreceptor precursors derived from three-dimensional embryonic stem cell cultures integrate and mature within adult degenerate retina. Nat. Biotechnol. 31, 741-747.

Gu,P., Harwood,L.J., Zhang,X., Wylie,M., Curry,W.J., and Cogliati,T. (2007). Isolation of retinal progenitor and stem cells from the porcine eye. Mol. Vis. 13, 1045-1057.

Gualdoni,S., Baron,M., Lakowski,J., Decembrini,S., Smith,A.J., Pearson,R.A., Ali,R.R., and Sowden,J.C. (2010). Adult ciliary epithelial cells, previously identified as retinal stem cells with potential for retinal repair, fail to differentiate into new rod photoreceptors. Stem Cells 28, 1048-1059.

Gumy,L.F., Tan,C.L., and Fawcett,J.W. (2010). The role of local protein synthesis and degradation in axon regeneration. Exp. Neurol. 223, 28-37.

Gust,J. and Reh,T.A. (2011). Adult donor rod photoreceptors integrate into the mature mouse retina. Invest Ophthalmol. Vis. Sci. 52, 5266-5272.

Haines,J.L., Hauser,M.A., Schmidt,S., Scott,W.K., Olson,L.M., Gallins,P., Spencer,K.L., Kwan,S.Y., Noureddine,M., Gilbert,J.R. et al. (2005). Complement factor H variant increases the risk of age-related macular degeneration. Science 308, 419-421.

Hamanaka,S., Yamaguchi,T., Kobayashi,T., Kato-Itoh,M., Yamazaki,S., Sato,H., Umino,A., Wakiyama,Y., Arai,M., Sanbo,M. et al. (2011). Generation of germlinecompetent rat induced pluripotent stem cells. PLoS. One. 6, e22008.

Harris,W.A. and Perron,M. (1998). Molecular recapitulation: the growth of the vertebrate retina. Int. J. Dev. Biol. 42, 299-304.

Hartong,D.T., Berson,E.L., and Dryja,T.P. (2006). Retinitis pigmentosa. Lancet 368, 17951809.

Haruta,M., Kosaka,M., Kanegae,Y., Saito,I., Inoue,T., Kageyama,R., Nishida,A., Honda,Y., and Takahashi,M. (2001). Induction of photoreceptor-specific phenotypes in adult mammalian iris tissue. Nat. Neurosci. 4, 1163-1164.

Hashimoto,H., Itoh,M., Yamanaka,Y., Yamashita,S., Shimizu,T., Solnica-Krezel,L., Hibi,M., and Hirano,T. (2000). Zebrafish Dkk1 functions in forebrain specification and axial mesendoderm formation. Dev. Biol. 217, 138-152. 
Hatakeyama,J. and Kageyama,R. (2004). Retinal cell fate determination and bHLH factors. Semin. Cell Dev. Biol. 15, 83-89.

Hayes,S., Nelson,B.R., Buckingham,B., and Reh,T.A. (2007). Notch signaling regulates regeneration in the avian retina. Dev. Biol. 312, 300-311.

Heisenberg,C.P., Houart,C., Take-Uchi,M., Rauch,G.J., Young,N., Coutinho,P., Masai,I., Caneparo,L., Concha,M.L., Geisler,R. et al. (2001). A mutation in the Gsk3-binding domain of zebrafish Masterblind/Axin1 leads to a fate transformation of telencephalon and eyes to diencephalon. Genes Dev. 15, 1427-1434.

Hemmati-Brivanlou,A., Kelly,O.G., and Melton,D.A. (1994). Follistatin, an antagonist of activin, is expressed in the Spemann organizer and displays direct neuralizing activity. Cell 77, 283-295.

Hendrickson,A., Bumsted-O'Brien,K., Natoli,R., Ramamurthy,V., Possin,D., and Provis,J. (2008). Rod photoreceptor differentiation in fetal and infant human retina. Exp. Eye Res. 87, 415-426.

Hennig,A.K., Peng,G.H., and Chen,S. (2008). Regulation of photoreceptor gene expression by Crx-associated transcription factor network. Brain Res. 1192, 114-133.

Hicks,D. and Courtois,Y. (1988). Acidic fibroblast growth factor stimulates opsin levels in retinal photoreceptor cells in vitro. FEBS Lett. 234, 475-479.

Hicks,D. and Courtois,Y. (1992). Fibroblast growth factor stimulates photoreceptor differentiation in vitro. J. Neurosci. 12, 2022-2033.

Hirami,Y., Osakada,F., Takahashi,K., Okita,K., Yamanaka,S., Ikeda,H., Yoshimura,N., and Takahashi,M. (2009). Generation of retinal cells from mouse and human induced pluripotent stem cells. Neurosci. Lett. 458, 126-131.

Hollenberg,M.J. and Spira,A.W. (1973). Human retinal development: ultrastructure of the outer retina. Am. J. Anat. 137, 357-385.

Holt,C.E., Bertsch,T.W., Ellis,H.M., and Harris,W.A. (1988). Cellular determination in the Xenopus retina is independent of lineage and birth date. Neuron 1, 15-26.

Homma,K., Okamoto,S., Mandai,M., Gotoh,N., Rajasimha,H.K., Chang,Y.S., Chen,S., Li,W., Cogliati,T., Swaroop,A. et al. (2013). Developing rods transplanted into the degenerating retina of Crx-knockout mice exhibit neural activity similar to native photoreceptors. Stem Cells 31, 1149-1159.

Hori,J., Ng,T.F., Shatos,M., Klassen,H., Streilein,J.W., and Young,M.J. (2003). Neural progenitor cells lack immunogenicity and resist destruction as allografts. Stem Cells 21, 405416.

Horsford,D.J., Nguyen,M.T., Sellar,G.C., Kothary,R., Arnheiter,H., and McInnes,R.R. (2005). Chx10 repression of Mitf is required for the maintenance of mammalian neuroretinal identity. Development 132, 177-187. 
Houart,C., Caneparo,L., Heisenberg,C., Barth,K., Take-Uchi,M., and Wilson,S. (2002). Establishment of the telencephalon during gastrulation by local antagonism of Wnt signaling. Neuron 35, 255-265.

Houart,C., Westerfield,M., and Wilson,S.W. (1998). A small population of anterior cells patterns the forebrain during zebrafish gastrulation. Nature 391, 788-792.

Hu,Q., Friedrich,A.M., Johnson,L.V., and Clegg,D.O. (2010). Memory in induced pluripotent stem cells: reprogrammed human retinal-pigmented epithelial cells show tendency for spontaneous redifferentiation. Stem Cells 28, 1981-1991.

Humayun,M.S., Dorn,J.D., Ahuja,A.K., Caspi,A., Filley,E., Dagnelie,G., Salzmann,J., Santos,A., Duncan,J., Dacruz,L. et al. (2009). Preliminary 6 month results from the Argus II epiretinal prosthesis feasibility study. Conf. Proc. IEEE Eng Med. Biol. Soc. 2009, 45664568.

Hyatt,G.A. and Dowling,J.E. (1997). Retinoic acid. A key molecule for eye and photoreceptor development. Invest Ophthalmol. Vis. Sci. 38, 1471-1475.

Hyatt,G.A., Schmitt,E.A., Fadool,J.M., and Dowling,J.E. (1996). Retinoic acid alters photoreceptor development in vivo. Proc. Natl. Acad. Sci. U. S. A 93, 13298-13303.

Ikeda,H., Osakada,F., Watanabe,K., Mizuseki,K., Haraguchi,T., Miyoshi,H., Kamiya,D., Honda,Y., Sasai,N., Yoshimura,N. et al. (2005). Generation of Rx+/Pax6+ neural retinal precursors from embryonic stem cells. Proc. Natl. Acad. Sci. U. S. A 102, 11331-11336.

Ilia,M. and Jeffery,G. (1999). Retinal mitosis is regulated by dopa, a melanin precursor that may influence the time at which cells exit the cell cycle: analysis of patterns of cell production in pigmented and albino retinae. J. Comp Neurol. 405, 394-405.

Inatani,M., Tanihara,H., Oohira,A., Otori,Y., Nishida,A., Honjo,M., Kido,N., and Honda,Y. (2000). Neuroglycan C, a neural tissue-specific transmembrane chondroitin sulfate proteoglycan, in retinal neural network formation. Invest Ophthalmol. Vis. Sci. 41, 4338-4346.

Inoue,T., Coles,B.L., Dorval,K., Bremner,R., Bessho,Y., Kageyama,R., Hino,S., Matsuoka,M., Craft,C.M., McInnes,R.R. et al. (2010). Maximizing functional photoreceptor differentiation from adult human retinal stem cells. Stem Cells 28, 489-500.

Inoue,Y., Yanagi,Y., Tamaki,Y., Uchida,S., Kawase,Y., Araie,M., and Okochi,H. (2005). Clonogenic analysis of ciliary epithelial derived retinal progenitor cells in rabbits. Exp. Eye Res. 81, 437-445.

Jacobson,S.G., Cideciyan,A.V., Ratnakaram,R., Heon,E., Schwartz,S.B., Roman,A.J., Peden,M.C., Aleman,T.S., Boye,S.L., Sumaroka,A. et al. (2012). Gene therapy for leber congenital amaurosis caused by RPE65 mutations: safety and efficacy in 15 children and adults followed up to 3 years. Arch. Ophthalmol. 130, 9-24. 
Jadhav,A.P., Mason,H.A., and Cepko,C.L. (2006). Notch 1 inhibits photoreceptor production in the developing mammalian retina. Development 133, 913-923.

Jager,R.D., Mieler,W.F., and Miller,J.W. (2008). Age-related macular degeneration. $N$. Engl. J. Med. 358, 2606-2617.

James,J., Das,A.V., Rahnenfuhrer,J., and Ahmad,I. (2004). Cellular and molecular characterization of early and late retinal stem cells/progenitors: differential regulation of proliferation and context dependent role of Notch signaling. J. Neurobiol. 61, 359-376.

Jasty,S., Srinivasan,P., Pasricha,G., Chatterjee,N., and Subramanian,K. (2012). Gene expression profiles and retinal potential of stem/progenitor cells derived from human iris and ciliary pigment epithelium. Stem Cell Rev. 8, 1163-1177.

Jayaram,H., Jones,M.F., Eastlake,K., Cottrill,P.B., Becker,S., Wiseman,J., Khaw,P.T., and Limb,G.A. (2014). Transplantation of photoreceptors derived from human Muller glia restore rod function in the P23H rat. Stem Cells Transl. Med. 3, 323-333.

Jensen,A.M. and Wallace,V.A. (1997). Expression of Sonic hedgehog and its putative role as a precursor cell mitogen in the developing mouse retina. Development 124, 363-371.

Jia,L., Oh,E.C., Ng,L., Srinivas,M., Brooks,M., Swaroop,A., and Forrest,D. (2009). Retinoid-related orphan nuclear receptor RORbeta is an early-acting factor in rod photoreceptor development. Proc. Natl. Acad. Sci. U. S. A 106, 17534-17539.

Jian,Q., Xu,H., Xie,H., Tian,C., Zhao,T., and Yin,Z. (2009). Activation of retinal stem cells in the proliferating marginal region of RCS rats during development of retinitis pigmentosa. Neurosci. Lett. 465, 41-44.

Jin,Z.B., Okamoto,S., Xiang,P., and Takahashi,M. (2012). Integration-free induced pluripotent stem cells derived from retinitis pigmentosa patient for disease modeling. Stem Cells Transl. Med. 1, 503-509.

Johns,P.R. and Easter,S.S., Jr. (1977). Growth of the adult goldfish eye. II. Increase in retinal cell number. J. Comp Neurol. 176, 331-341.

Jones,B.W. and Marc,R.E. (2005). Retinal remodeling during retinal degeneration. Exp. Eye Res. 81, 123-137.

Jones,B.W., Watt,C.B., Frederick,J.M., Baehr,W., Chen,C.K., Levine,E.M., Milam,A.H., LaVail,M.M., and Marc,R.E. (2003). Retinal remodeling triggered by photoreceptor degenerations. J. Comp Neurol. 464, 1-16.

Kaneko,Y., Sakakibara,S., Imai,T., Suzuki,A., Nakamura,Y., Sawamoto,K., Ogawa,Y., Toyama,Y., Miyata,T., and Okano,H. (2000). Musashi1: an evolutionally conserved marker for CNS progenitor cells including neural stem cells. Dev. Neurosci. 22, 139-153.

Karl,M.O., Hayes,S., Nelson,B.R., Tan,K., Buckingham,B., and Reh,T.A. (2008). Stimulation of neural regeneration in the mouse retina. Proc. Natl. Acad. Sci. U. S. A 105, 19508-19513. 
Kassen,S.C., Ramanan,V., Montgomery,J.E., Burket,T., Liu,C.G., Vihtelic,T.S., and Hyde,D.R. (2007). Time course analysis of gene expression during light-induced photoreceptor cell death and regeneration in albino zebrafish. Dev. Neurobiol. 67, 10091031.

Katoh,K., Omori,Y., Onishi,A., Sato,S., Kondo,M., and Furukawa,T. (2010). Blimp1 suppresses Chx10 expression in differentiating retinal photoreceptor precursors to ensure proper photoreceptor development. J. Neurosci. 30, 6515-6526.

Kawasaki,H., Mizuseki,K., Nishikawa,S., Kaneko,S., Kuwana,Y., Nakanishi,S., Nishikawa,S.I., and Sasai,Y. (2000). Induction of midbrain dopaminergic neurons from ES cells by stromal cell-derived inducing activity. Neuron 28, 31-40.

Kazanskaya,O., Glinka,A., and Niehrs,C. (2000). The role of Xenopus dickkopf1 in prechordal plate specification and neural patterning. Development 127, 4981-4992.

Kim,D., Kim,C.H., Moon,J.I., Chung,Y.G., Chang,M.Y., Han,B.S., Ko,S., Yang,E., Cha,K.Y., Lanza,R. et al. (2009). Generation of human induced pluripotent stem cells by direct delivery of reprogramming proteins. Cell Stem Cell 4, 472-476.

Kim,K., Doi,A., Wen,B., Ng,K., Zhao,R., Cahan,P., Kim,J., Aryee,M.J., Ji,H., Ehrlich,L.I. et al. (2010). Epigenetic memory in induced pluripotent stem cells. Nature 467, 285-290.

Kim,S.U. (2007). Genetically engineered human neural stem cells for brain repair in neurological diseases. Brain Dev. 29, 193-201.

Klassen,H., Schwartz,P.H., Ziaeian,B., Nethercott,H., Young,M.J., Bragadottir,R., Tullis,G.E., Warfvinge,K., and Narfstrom,K. (2007). Neural precursors isolated from the developing cat brain show retinal integration following transplantation to the retina of the dystrophic cat. Vet. Ophthalmol. 10, 245-253.

Klassen,H.J., Ng,T.F., Kurimoto,Y., Kirov,I., Shatos,M., Coffey,P., and Young,M.J. (2004). Multipotent retinal progenitors express developmental markers, differentiate into retinal neurons, and preserve light-mediated behavior. Invest Ophthalmol. Vis. Sci. 45, 41674173.

Klein,R.J., Zeiss,C., Chew,E.Y., Tsai,J.Y., Sackler,R.S., Haynes,C., Henning,A.K., SanGiovanni,J.P., Mane,S.M., Mayne,S.T. et al. (2005). Complement factor H polymorphism in age-related macular degeneration. Science 308, 385-389.

Koberlein,J., Beifus,K., Schaffert,C., and Finger,R.P. (2013). The economic burden of visual impairment and blindness: a systematic review. BMJ Open. 3, e003471.

Kohno,H., Sakai,T., and Kitahara,K. (2006). Induction of nestin, Ki-67, and cyclin D1 expression in Muller cells after laser injury in adult rat retina. Graefes Arch. Clin. Exp. Ophthalmol. 244, 90-95. 
Koike,C., Nishida,A., Ueno,S., Saito,H., Sanuki,R., Sato,S., Furukawa,A., Aizawa,S., Matsuo,I., Suzuki,N. et al. (2007). Functional roles of Otx2 transcription factor in postnatal mouse retinal development. Mol. Cell Biol. 27, 8318-8329.

Kokkinopoulos,I., Pearson,R.A., Macneil,A., Dhomen,N.S., MacLaren,R.E., Ali,R.R., and Sowden,J.C. (2008). Isolation and characterisation of neural progenitor cells from the adult Chx10(orJ/orJ) central neural retina. Mol. Cell Neurosci. 38, 359-373.

Kralj-Hans,I., Tibber,M., Jeffery,G., and Mobbs,P. (2006). Differential effect of dopamine on mitosis in early postnatal albino and pigmented rat retinae. J. Neurobiol. 66, 47-55.

Kubota,R., Hokoc,J.N., Moshiri,A., McGuire,C., and Reh,T.A. (2002). A comparative study of neurogenesis in the retinal ciliary marginal zone of homeothermic vertebrates. Brain Res. Dev. Brain Res. 134, 31-41.

Kurosawa,H. (2007). Methods for inducing embryoid body formation: in vitro differentiation system of embryonic stem cells. J. Biosci. Bioeng. 103, 389-398.

Kwan,A.S., Wang,S., and Lund,R.D. (1999). Photoreceptor layer reconstruction in a rodent model of retinal degeneration. Exp. Neurol. 159, 21-33.

Lakowski,J., Baron,M., Bainbridge,J., Barber,A.C., Pearson,R.A., Ali,R.R., and Sowden,J.C. (2010). Cone and rod photoreceptor transplantation in models of the childhood retinopathy Leber congenital amaurosis using flow-sorted Crx-positive donor cells. Hum. Mol. Genet. 19, 4545-4559.

Lakowski,J., Han,Y.T., Pearson,R.A., Gonzalez-Cordero,A., West,E.L., Gualdoni,S., Barber,A.C., Hubank,M., Ali,R.R., and Sowden,J.C. (2011). Effective transplantation of photoreceptor precursor cells selected via cell surface antigen expression. Stem Cells 29, 1391-1404.

Lamba,D.A., Gust,J., and Reh,T.A. (2009). Transplantation of human embryonic stem cellderived photoreceptors restores some visual function in Crx-deficient mice. Cell Stem Cell 4, 73-79.

Lamba,D.A., Karl,M.O., Ware,C.B., and Reh,T.A. (2006). Efficient generation of retinal progenitor cells from human embryonic stem cells. Proc. Natl. Acad. Sci. U. S. A 103, 12769-12774.

Lamba,D.A., McUsic,A., Hirata,R.K., Wang,P.R., Russell,D., and Reh,T.A. (2010). Generation, purification and transplantation of photoreceptors derived from human induced pluripotent stem cells. PLoS. One. 5, e8763.

Lawrence,J.M., Singhal,S., Bhatia,B., Keegan,D.J., Reh,T.A., Luthert,P.J., Khaw,P.T., and Limb,G.A. (2007). MIO-M1 cells and similar muller glial cell lines derived from adult human retina exhibit neural stem cell characteristics. Stem Cells 25, 2033-2043. 
Le,T.T., Wroblewski,E., Patel,S., Riesenberg,A.N., and Brown,N.L. (2006). Math5 is required for both early retinal neuron differentiation and cell cycle progression. Dev. Biol. 295, 764-778.

Leaver,S.G., Cui,Q., Plant,G.W., Arulpragasam,A., Hisheh,S., Verhaagen,J., and Harvey,A.R. (2006). AAV-mediated expression of CNTF promotes long-term survival and regeneration of adult rat retinal ganglion cells. Gene Ther. 13, 1328-1341.

Lee,E.J., Kim,I.B., Lee,E., Kwon,S.O., Oh,S.J., and Chun,M.H. (2003). Differential expression and cellular localization of doublecortin in the developing rat retina. Eur. J. Neurosci. 17, 1542-1548.

Lee,S.H., Lumelsky,N., Studer,L., Auerbach,J.M., and McKay,R.D. (2000). Efficient generation of midbrain and hindbrain neurons from mouse embryonic stem cells. Nat. Biotechnol. 18, 675-679.

Lendahl,U., Zimmerman,L.B., and McKay,R.D. (1990). CNS stem cells express a new class of intermediate filament protein. Cell 60, 585-595.

Lenkowski,J.R., Qin,Z., Sifuentes,C.J., Thummel,R., Soto,C.M., Moens,C.B., and Raymond,P.A. (2013). Retinal regeneration in adult zebrafish requires regulation of TGFbeta signaling. Glia 61, 1687-1697.

Lenkowski,J.R. and Raymond,P.A. (2014). Muller glia: Stem cells for generation and regeneration of retinal neurons in teleost fish. Prog. Retin. Eye Res. 40, 94-123.

Levine,E.M., Roelink,H., Turner,J., and Reh,T.A. (1997a). Sonic hedgehog promotes rod photoreceptor differentiation in mammalian retinal cells in vitro. J. Neurosci. 17, 6277-6288.

Levine,E.M., Roelink,H., Turner,J., and Reh,T.A. (1997b). Sonic hedgehog promotes rod photoreceptor differentiation in mammalian retinal cells in vitro. J. Neurosci. 17, 6277-6288.

Lillien,L. (1995). Changes in retinal cell fate induced by overexpression of EGF receptor. Nature 377, 158-162.

Lillien,L. and Cepko,C. (1992). Control of proliferation in the retina: temporal changes in responsiveness to FGF and TGF alpha. Development 115, 253-266.

Linker,C. and Stern,C.D. (2004). Neural induction requires BMP inhibition only as a late step, and involves signals other than FGF and Wnt antagonists. Development 131, 56715681 .

Liu,B., Hunter,D.J., Rooker,S., Chan,A., Paulus,Y.M., Leucht,P., Nusse,Y., Nomoto,H., and Helms,J.A. (2013). Wnt signaling promotes Muller cell proliferation and survival after injury. Invest Ophthalmol. Vis. Sci. 54, 444-453.

Locker,M., Borday,C., and Perron,M. (2009). Stemness or not stemness? Current status and perspectives of adult retinal stem cells. Curr. Stem Cell Res. Ther. 4, 118-130.

Locker,M., EI,Y.W., Mazurier,N., Dullin,J.P., and Perron,M. (2010). A decade of mammalian retinal stem cell research. Arch. Ital. Biol. 148, 59-72. 
Loh,Y.H., Agarwal,S., Park,I.H., Urbach,A., Huo,H., Heffner,G.C., Kim,K., Miller,J.D., Ng,K., and Daley,G.Q. (2009). Generation of induced pluripotent stem cells from human blood. Blood 113, 5476-5479.

Lolley,R.N., Rong,H., and Craft,C.M. (1994). Linkage of photoreceptor degeneration by apoptosis with inherited defect in phototransduction. Invest Ophthalmol. Vis. Sci. 35, 358362.

Ma,N. and Streilein,J.W. (1998). Contribution of microglia as passenger leukocytes to the fate of intraocular neuronal retinal grafts. Invest Ophthalmol. Vis. Sci. 39, 2384-2393.

Ma,Y., Liu,M., and Li,Y. (2011). Secretion of bacterial chondroitinase ABC from bone marrow stromal cells by glycosylation site mutation: a promising approach for axon regeneration. Med. Hypotheses 77, 914-916.

MacLaren,R.E., Pearson,R.A., Macneil,A., Douglas,R.H., Salt,T.E., Akimoto,M., Swaroop,A., Sowden,J.C., and Ali,R.R. (2006). Retinal repair by transplantation of photoreceptor precursors. Nature 444, 203-207.

Macneil,A., Pearson,R.A., MacLaren,R.E., Smith,A.J., Sowden,J.C., and Ali,R.R. (2007). Comparative analysis of progenitor cells isolated from the iris, pars plana, and ciliary body of the adult porcine eye. Stem Cells 25, 2430-2438.

Maguire,A.M., Simonelli,F., Pierce,E.A., Pugh,E.N., Jr., Mingozzi,F., Bennicelli,J., Banfi,S., Marshall,K.A., Testa,F., Surace,E.M. et al. (2008). Safety and efficacy of gene transfer for Leber's congenital amaurosis. N. Engl. J. Med. 358, 2240-2248.

Maherali,N., Sridharan,R., Xie,W., Utikal,J., Eminli,S., Arnold,K., Stadtfeld,M., Yachechko,R., Tchieu,J., Jaenisch,R. et al. (2007). Directly reprogrammed fibroblasts show global epigenetic remodeling and widespread tissue contribution. Cell Stem Cell 1, 5570.

Man,H.X., Godlewski,S., and Hamard,H. (1964). [Histopathological study of the eyeballs and orbital contents in a case of leukosis of late hematologic revelation which was expressed by various neurologic manifestations with ocular involvement and especially of papilledema]. Bull. Soc. Ophtalmol. Fr. 64, 527-535.

Marquardt,T. (2003). Transcriptional control of neuronal diversification in the retina. Prog. Retin. Eye Res. 22, 567-577.

Marquardt,T. and Gruss,P. (2002). Generating neuronal diversity in the retina: one for nearly all. Trends Neurosci. 25, 32-38.

Martin,G.R. (1981). Isolation of a pluripotent cell line from early mouse embryos cultured in medium conditioned by teratocarcinoma stem cells. Proc. Natl. Acad. Sci. U. S. A 78, 76347638. 
Martinez-Navarrete,G.C., Angulo,A., Martin-Nieto,J., and Cuenca,N. (2008). Gradual morphogenesis of retinal neurons in the peripheral retinal margin of adult monkeys and humans. J. Comp Neurol. 511, 557-580.

Mason,I. (2007). Initiation to end point: the multiple roles of fibroblast growth factors in neural development. Nat. Rev. Neurosci. 8, 583-596.

Mayer,E.J., Carter,D.A., Ren,Y., Hughes,E.H., Rice,C.M., Halfpenny,C.A., Scolding,N.J., and Dick,A.D. (2005). Neural progenitor cells from postmortem adult human retina. Br. J. Ophthalmol. 89, 102-106.

Mazzoni,F., Novelli,E., and Strettoi,E. (2008). Retinal ganglion cells survive and maintain normal dendritic morphology in a mouse model of inherited photoreceptor degeneration. J. Neurosci. 28, 14282-14292.

Mears,A.J., Kondo,M., Swain,P.K., Takada,Y., Bush,R.A., Saunders,T.L., Sieving,P.A., and Swaroop,A. (2001a). Nrl is required for rod photoreceptor development. Nat. Genet. 29, 447-452.

Mears,A.J., Kondo,M., Swain,P.K., Takada,Y., Bush,R.A., Saunders,T.L., Sieving,P.A., and Swaroop,A. (2001b). Nrl is required for rod photoreceptor development. Nat. Genet. 29, 447-452.

Mellough,C.B., Cui,Q., and Harvey,A.R. (2007). Treatment of adult neural progenitor cells prior to transplantation affects graft survival and integration in a neonatal and adult rat model of selective retinal ganglion cell depletion. Restor. Neurol. Neurosci. 25, 177-190.

Mellough,C.B., Sernagor,E., Moreno-Gimeno,I., Steel,D.H., and Lako,M. (2012). Efficient stage-specific differentiation of human pluripotent stem cells toward retinal photoreceptor cells. Stem Cells 30, 673-686.

Meyer,J.S., Howden,S.E., Wallace,K.A., Verhoeven,A.D., Wright,L.S., Capowski,E.E., Pinilla,I., Martin,J.M., Tian,S., Stewart,R. et al. (2011). Optic vesicle-like structures derived from human pluripotent stem cells facilitate a customized approach to retinal disease treatment. Stem Cells 29, 1206-1218.

Meyer,J.S., Shearer,R.L., Capowski,E.E., Wright,L.S., Wallace,K.A., McMillan,E.L., Zhang,S.C., and Gamm,D.M. (2009). Modeling early retinal development with human embryonic and induced pluripotent stem cells. Proc. Natl. Acad. Sci. U. S. A 106, 1669816703.

Meyers,J.R., Hu,L., Moses,A., Kaboli,K., Papandrea,A., and Raymond,P.A. (2012). betacatenin/Wnt signaling controls progenitor fate in the developing and regenerating zebrafish retina. Neural Dev. 7, 30.

Minassian,D.C., Reidy,A., Lightstone,A., and Desai,P. (2011). Modelling the prevalence of age-related macular degeneration (2010-2020) in the UK: expected impact of antivascular endothelial growth factor (VEGF) therapy. Br. J. Ophthalmol. 95, 1433-1436. 
Mizuseki,K., Sakamoto,T., Watanabe,K., Muguruma,K., Ikeya,M., Nishiyama,A., Arakawa,A., Suemori,H., Nakatsuji,N., Kawasaki,H. et al. (2003). Generation of neural crest-derived peripheral neurons and floor plate cells from mouse and primate embryonic stem cells. Proc. Natl. Acad. Sci. U. S. A 100, 5828-5833.

Moe,M.C., Kolberg,R.S., Sandberg,C., Vik-Mo,E., Olstorn,H., Varghese,M., Langmoen,I.A., and Nicolaissen,B. (2009). A comparison of epithelial and neural properties in progenitor cells derived from the adult human ciliary body and brain. Exp. Eye Res. 88, 30-38.

Morrow,E.M., Belliveau,M.J., and Cepko,C.L. (1998). Two phases of rod photoreceptor differentiation during rat retinal development. J. Neurosci. 18, 3738-3748.

Moshiri,A., Close,J., and Reh,T.A. (2004). Retinal stem cells and regeneration. Int. J. Dev. Biol. 48, 1003-1014.

Moshiri,A. and Reh,T.A. (2004). Persistent progenitors at the retinal margin of ptc+/- mice. J. Neurosci. 24, 229-237.

Mukhopadhyay,M., Shtrom,S., Rodriguez-Esteban,C., Chen,L., Tsukui,T., Gomer,L., Dorward,D.W., Glinka,A., Grinberg,A., Huang,S.P. et al. (2001). Dickkopf1 is required for embryonic head induction and limb morphogenesis in the mouse. Dev. Cell 1, 423-434.

Nagashima,M., Barthel,L.K., and Raymond,P.A. (2013). A self-renewing division of zebrafish Muller glial cells generates neuronal progenitors that require $\mathrm{N}$-cadherin to regenerate retinal neurons. Development 140, 4510-4521.

Nakamura,K. and Chiba,C. (2007). Evidence for Notch signaling involvement in retinal regeneration of adult newt. Brain Res. 1136, 28-42.

Nakano,T., Ando,S., Takata,N., Kawada,M., Muguruma,K., Sekiguchi,K., Saito,K., Yonemura,S., Eiraku,M., and Sasai,Y. (2012). Self-formation of optic cups and storable stratified neural retina from human ESCs. Cell Stem Cell 10, 771-785.

Nathans,J., Thomas,D., and Hogness,D.S. (1986). Molecular genetics of human color vision: the genes encoding blue, green, and red pigments. Science 232, 193-202.

Nelson,C.M., Gorsuch,R.A., Bailey,T.J., Ackerman,K.M., Kassen,S.C., and Hyde,D.R. (2012). Stat3 defines three populations of Muller glia and is required for initiating maximal muller glia proliferation in the regenerating zebrafish retina. J. Comp Neurol. 520, 42944311.

Newman,E. and Reichenbach,A. (1996). The Muller cell: a functional element of the retina. Trends Neurosci. 19, 307-312.

Ng,L., Hurley,J.B., Dierks,B., Srinivas,M., Salto,C., Vennstrom,B., Reh,T.A., and Forrest,D. (2001a). A thyroid hormone receptor that is required for the development of green cone photoreceptors. Nat. Genet. 27, 94-98. 
Ng,L., Hurley,J.B., Dierks,B., Srinivas,M., Salto,C., Vennstrom,B., Reh,T.A., and Forrest,D. (2001b). A thyroid hormone receptor that is required for the development of green cone photoreceptors. Nat. Genet. 27, 94-98.

Nguyen,M. and Arnheiter,H. (2000). Signaling and transcriptional regulation in early mammalian eye development: a link between FGF and MITF. Development 127, 3581-3591. Nickerson,P.E., Emsley,J.G., Myers,T., and Clarke,D.B. (2007). Proliferation and expression of progenitor and mature retinal phenotypes in the adult mammalian ciliary body after retinal ganglion cell injury. Invest Ophthalmol. Vis. Sci. 48, 5266-5275.

Niehrs,C. (2001). The Spemann organizer and embryonic head induction. EMBO J. 20, 631 637.

Nishida,A., Furukawa,A., Koike,C., Tano,Y., Aizawa,S., Matsuo,I., and Furukawa,T. (2003). Otx2 homeobox gene controls retinal photoreceptor cell fate and pineal gland development. Nat. Neurosci. 6, 1255-1263.

Nishida,A., Takahashi,M., Tanihara,H., Nakano,I., Takahashi,J.B., Mizoguchi,A., Ide,C., and Honda,Y. (2000). Incorporation and differentiation of hippocampus-derived neural stem cells transplanted in injured adult rat retina. Invest Ophthalmol. Vis. Sci. 41, 4268-4274.

Nishiguchi,K.M., Friedman,J.S., Sandberg,M.A., Swaroop,A., Berson,E.L., and Dryja,T.P. (2004). Recessive NRL mutations in patients with clumped pigmentary retinal degeneration and relative preservation of blue cone function. Proc. Natl. Acad. Sci. U. S. A 101, 17819-17824.

Nishiguchi,K.M., Kaneko,H., Nakamura,M., Kachi,S., and Terasaki,H. (2008). Identification of photoreceptor precursors in the pars plana during ocular development and after retinal injury. Invest Ophthalmol. Vis. Sci. 49, 422-428.

Nordstrom,U., Jessell,T.M., and Edlund,T. (2002). Progressive induction of caudal neural character by graded Wnt signaling. Nat. Neurosci. 5, 525-532.

O'Brien,K.M., Schulte,D., and Hendrickson,A.E. (2003). Expression of photoreceptorassociated molecules during human fetal eye development. Mol. Vis. 9, 401-409.

O'Rahilly,R. (1975). The prenatal development of the human eye. Exp. Eye Res. 21, 93112.

O'Rahilly,R., Muller,F., and Bossy,J. (1982). [Atlas of the stages of development of the nervous system in the intact human embryo]. Arch. Anat. Histol. Embryol. 65, 57-76.

Oh,E.C., Cheng,H., Hao,H., Jia,L., Khan,N.W., and Swaroop,A. (2008). Rod differentiation factor NRL activates the expression of nuclear receptor NR2E3 to suppress the development of cone photoreceptors. Brain Res. 1236, 16-29.

Okita,K., Ichisaka,T., and Yamanaka,S. (2007). Generation of germline-competent induced pluripotent stem cells. Nature 448, 313-317. 
Ooto,S., Akagi,T., Kageyama,R., Akita,J., Mandai,M., Honda,Y., and Takahashi,M. (2004). Potential for neural regeneration after neurotoxic injury in the adult mammalian retina. Proc. Natl. Acad. Sci. U. S. A 101, 13654-13659.

Opas,M., Davies,J.R., Zhou,Y., and Dziak,E. (2001). Formation of retinal pigment epithelium in vitro by transdifferentiation of neural retina cells. Int. J. Dev. Biol. 45, 633-642.

Osakada,F., Ikeda,H., Mandai,M., Wataya,T., Watanabe,K., Yoshimura,N., Akaike,A., Sasai,Y., and Takahashi,M. (2008). Toward the generation of rod and cone photoreceptors from mouse, monkey and human embryonic stem cells. Nat. Biotechnol. 26, 215-224.

Osakada,F., Ikeda,H., Sasai,Y., and Takahashi,M. (2009a). Stepwise differentiation of pluripotent stem cells into retinal cells. Nat. Protoc. 4, 811-824.

Osakada,F., Jin,Z.B., Hirami,Y., Ikeda,H., Danjyo,T., Watanabe,K., Sasai,Y., and Takahashi,M. (2009b). In vitro differentiation of retinal cells from human pluripotent stem cells by small-molecule induction. J. Cell Sci. 122, 3169-3179.

Otteson,D.C., Cirenza,P.F., and Hitchcock,P.F. (2002). Persistent neurogenesis in the teleost retina: evidence for regulation by the growth-hormone/insulin-like growth factor-I axis. Mech. Dev. 117, 137-149.

Owen,C.G., Jarrar,Z., Wormald,R., Cook,D.G., Fletcher,A.E., and Rudnicka,A.R. (2012). The estimated prevalence and incidence of late stage age related macular degeneration in the UK. Br. J. Ophthalmol. 96, 752-756.

Paek,H., Gutin,G., and Hebert,J.M. (2009). FGF signaling is strictly required to maintain early telencephalic precursor cell survival. Development 136, 2457-2465.

Panagis,L., Thanos,S., Fischer,D., and Dermon,C.R. (2005). Unilateral optic nerve crush induces bilateral retinal glial cell proliferation. Eur. J. Neurosci. 21, 2305-2309.

Parain,K., Mazurier,N., Bronchain,O., Borday,C., Cabochette,P., Chesneau,A., Colozza,G., EI,Y.W., Hamdache,J., Locker,M. et al. (2012). A large scale screen for neural stem cell markers in Xenopus retina. Dev. Neurobiol. 72, 491-506.

Parameswaran,S., Balasubramanian,S., Babai,N., Qiu,F., Eudy,J.D., Thoreson,W.B., and Ahmad,I. (2010). Induced pluripotent stem cells generate both retinal ganglion cells and photoreceptors: therapeutic implications in degenerative changes in glaucoma and agerelated macular degeneration. Stem Cells 28, 695-703.

Pearson,R.A. (2014). Advances in repairing the degenerate retina by rod photoreceptor transplantation. Biotechnol. Adv. 32, 485-491.

Pearson,R.A., Barber,A.C., Rizzi,M., Hippert,C., Xue,T., West,E.L., Duran,Y., Smith,A.J., Chuang,J.Z., Azam,S.A. et al. (2012). Restoration of vision after transplantation of photoreceptors. Nature 485, 99-103.

Pearson,R.A., Barber,A.C., West,E.L., MacLaren,R.E., Duran,Y., Bainbridge,J.W., Sowden,J.C., and Ali,R.R. (2010). Targeted disruption of outer limiting membrane 
junctional proteins (Crb1 and ZO-1) increases integration of transplanted photoreceptor precursors into the adult wild-type and degenerating retina. Cell Transplant. 19, 487-503.

Pearson,R.A., Hippert,C., Graca,A.B., and Barber,A.C. (2014). Photoreceptor replacement therapy: Challenges presented by the diseased recipient retinal environment. Vis. Neurosci. 1-12.

Peng,G.H., Ahmad,O., Ahmad,F., Liu,J., and Chen,S. (2005). The photoreceptor-specific nuclear receptor $\mathrm{Nr} 2 \mathrm{e} 3$ interacts with $\mathrm{Crx}$ and exerts opposing effects on the transcription of rod versus cone genes. Hum. Mol. Genet. 14, 747-764.

Perron,M. and Harris,W.A. (2000). Retinal stem cells in vertebrates. Bioessays 22, 685688.

Perron,M., Kanekar,S., Vetter,M.L., and Harris,W.A. (1998). The genetic sequence of retinal development in the ciliary margin of the Xenopus eye. Dev. Biol. 199, 185-200.

Pevny,L.H., Sockanathan,S., Placzek,M., and Lovell-Badge,R. (1998). A role for SOX1 in neural determination. Development 125, 1967-1978.

Phillips,M.J., Wallace,K.A., Dickerson,S.J., Miller,M.J., Verhoeven,A.D., Martin,J.M., Wright,L.S., Shen,W., Capowski,E.E., Percin,E.F. et al. (2012). Blood-derived human iPS cells generate optic vesicle-like structures with the capacity to form retinal laminae and develop synapses. Invest Ophthalmol. Vis. Sci. 53, 2007-2019.

Pollak,J., Wilken,M.S., Ueki,Y., Cox,K.E., Sullivan,J.M., Taylor,R.J., Levine,E.M., and Reh,T.A. (2013). ASCL1 reprograms mouse Muller glia into neurogenic retinal progenitors. Development 140, 2619-2631.

Qin,Z., Barthel,L.K., and Raymond,P.A. (2009). Genetic evidence for shared mechanisms of epimorphic regeneration in zebrafish. Proc. Natl. Acad. Sci. U. S. A 106, 9310-9315.

Qiu,G., Seiler,M.J., Mui,C., Arai,S., Aramant,R.B., de Juan E Jr, and Sadda,S. (2005). Photoreceptor differentiation and integration of retinal progenitor cells transplanted into transgenic rats. Exp. Eye Res. 80, 515-525.

Radtke,N.D., Aramant,R.B., Petry,H.M., Green,P.T., Pidwell,D.J., and Seiler,M.J. (2008). Vision improvement in retinal degeneration patients by implantation of retina together with retinal pigment epithelium. Am. J. Ophthalmol. 146, 172-182.

Ragge,N.K., Brown,A.G., Poloschek,C.M., Lorenz,B., Henderson,R.A., Clarke,M.P., Russell-Eggitt,l., Fielder,A., Gerrelli,D., Martinez-Barbera,J.P. et al. (2005). Heterozygous mutations of OTX2 cause severe ocular malformations. Am. J. Hum. Genet. 76, 1008-1022.

Ramachandran,R., Fausett,B.V., and Goldman,D. (2010a). Ascl1a regulates Muller glia dedifferentiation and retinal regeneration through a Lin-28-dependent, let-7 microRNA signalling pathway. Nat. Cell Biol. 12, 1101-1107. 
Ramachandran,R., Reifler,A., Parent,J.M., and Goldman,D. (2010b). Conditional gene expression and lineage tracing of tuba1a expressing cells during zebrafish development and retina regeneration. J. Comp Neurol. 518, 4196-4212.

Ramachandran,R., Zhao,X.F., and Goldman,D. (2011). Ascl1a/Dkk/beta-catenin signaling pathway is necessary and glycogen synthase kinase-3beta inhibition is sufficient for zebrafish retina regeneration. Proc. Natl. Acad. Sci. U. S. A 108, 15858-15863.

Ramachandran,R., Zhao,X.F., and Goldman,D. (2012). Insm1a-mediated gene repression is essential for the formation and differentiation of Muller glia-derived progenitors in the injured retina. Nat. Cell Biol. 14, 1013-1023.

Rapaport,D.H., Wong,L.L., Wood,E.D., Yasumura,D., and LaVail,M.M. (2004). Timing and topography of cell genesis in the rat retina. J. Comp Neurol. 474, 304-324.

Raymond,P.A., Barthel,L.K., Bernardos,R.L., and Perkowski,J.J. (2006). Molecular characterization of retinal stem cells and their niches in adult zebrafish. BMC. Dev. Biol. 6, 36.

Rebagliati,M.R., Toyama,R., Haffter,P., and Dawid,I.B. (1998). cyclops encodes a nodalrelated factor involved in midline signaling. Proc. Natl. Acad. Sci. U. S. A 95, 9932-9937.

Reh,T.A. (1987). Cell-specific regulation of neuronal production in the larval frog retina. J. Neurosci. 7, 3317-3324.

Reh,T.A., Nagy,T., and Gretton,H. (1987). Retinal pigmented epithelial cells induced to transdifferentiate to neurons by laminin. Nature 330, 68-71.

Reh,T.A. and Pittack,C. (1995). Transdifferentiation and retinal regeneration. Semin. Cell Biol. 6, 137-142.

Reh,T.A. and Tully,T. (1986). Regulation of tyrosine hydroxylase-containing amacrine cell number in larval frog retina. Dev. Biol. 114, 463-469.

Reichman,S., Terray,A., Slembrouck,A., Nanteau,C., Orieux,G., Habeler,W., Nandrot,E.F., Sahel,J.A., Monville,C., and Goureau,O. (2014). From confluent human iPS cells to self-forming neural retina and retinal pigmented epithelium. Proc. Natl. Acad. Sci. U. S. A 111, 8518-8523.

Rhee,K.D., Nusinowitz,S., Chao,K., Yu,F., Bok,D., and Yang,X.J. (2013). CNTF-mediated protection of photoreceptors requires initial activation of the cytokine receptor gp130 in Muller glial cells. Proc. Natl. Acad. Sci. U. S. A 110, E4520-E4529.

Rhodes,R.H. (1979). A light microscopic study of the developing human neural retina. Am. J. Anat. 154, 195-209.

Richard-Parpaillon,L., Heligon,C., Chesnel,F., Boujard,D., and Philpott,A. (2002). The IGF pathway regulates head formation by inhibiting Wnt signaling in Xenopus. Dev. Biol. 244, 407-417. 
Rivolta,C., Berson,E.L., and Dryja,T.P. (2001). Dominant Leber congenital amaurosis, cone-rod degeneration, and retinitis pigmentosa caused by mutant versions of the transcription factor CRX. Hum. Mutat. 18, 488-498.

Roberts,M.R., Hendrickson,A., McGuire,C.R., and Reh,T.A. (2005). Retinoid X receptor (gamma) is necessary to establish the S-opsin gradient in cone photoreceptors of the developing mouse retina. Invest Ophthalmol. Vis. Sci. 46, 2897-2904.

Roberts,M.R., Srinivas,M., Forrest,D., Morreale de,E.G., and Reh,T.A. (2006). Making the gradient: thyroid hormone regulates cone opsin expression in the developing mouse retina. Proc. Natl. Acad. Sci. U. S. A 103, 6218-6223.

Rodieck,R.W. (1998). The First Steps in Seeing. Sinauer Associates Inc.,U.S.

Roesch,K., Jadhav,A.P., Trimarchi,J.M., Stadler,M.B., Roska,B., Sun,B.B., and Cepko,C.L. (2008). The transcriptome of retinal Muller glial cells. J. Comp Neurol. 509, 225238.

Rogers,C.D., Moody,S.A., and Casey,E.S. (2009). Neural induction and factors that stabilize a neural fate. Birth Defects Res. C. Embryo. Today 87, 249-262.

Ronn,L.C., Hartz,B.P., and Bock,E. (1998). The neural cell adhesion molecule (NCAM) in development and plasticity of the nervous system. Exp. Gerontol. 33, 853-864.

Rowan,S., Chen,C.M., Young,T.L., Fisher,D.E., and Cepko,C.L. (2004). Transdifferentiation of the retina into pigmented cells in ocular retardation mice defines a new function of the homeodomain gene Chx10. Development 131, 5139-5152.

Rutishauser,U. (1992). NCAM and its polysialic acid moiety: a mechanism for pull/push regulation of cell interactions during development? Dev. Supp/99-104.

Sakaguchi,D.S., Van Hoffelen,S.J., Grozdanic,S.D., Kwon,Y.H., Kardon,R.H., and Young,M.J. (2005). Neural progenitor cell transplants into the developing and mature central nervous system. Ann. N. Y. Acad. Sci. 1049, 118-134.

Sakaguchi,D.S., Van Hoffelen,S.J., Theusch,E., Parker,E., Orasky,J., Harper,M.M., Benediktsson,A., and Young,M.J. (2004). Transplantation of neural progenitor cells into the developing retina of the Brazilian opossum: an in vivo system for studying stem/progenitor cell plasticity. Dev. Neurosci. 26, 336-345.

Sakaguchi,D.S., Van Hoffelen,S.J., and Young,M.J. (2003). Differentiation and morphological integration of neural progenitor cells transplanted into the developing mammalian eye. Ann. N. Y. Acad. Sci. 995, 127-139.

Sakuma,R., Ohnishi,Y.Y., Meno,C., Fujii,H., Juan,H., Takeuchi,J., Ogura,T., Li,E., Miyazono,K., and Hamada,H. (2002). Inhibition of Nodal signalling by Lefty mediated through interaction with common receptors and efficient diffusion. Genes Cells 7, 401-412. 
Salero,E., Blenkinsop,T.A., Corneo,B., Harris,A., Rabin,D., Stern,J.H., and Temple,S. (2012). Adult human RPE can be activated into a multipotent stem cell that produces mesenchymal derivatives. Cell Stem Cell 10, 88-95.

Sam,T.N., Xiao,J., Roehrich,H., Low,W.C., and Gregerson,D.S. (2006). Engrafted neural progenitor cells express a tissue-restricted reporter gene associated with differentiated retinal photoreceptor cells. Cell Transplant. 15, 147-160.

Sanges,D., Romo,N., Simonte,G., Di,V.U., Tahoces,A.D., Fernandez,E., and Cosma,M.P. (2013). Wnt/beta-catenin signaling triggers neuron reprogramming and regeneration in the mouse retina. Cell Rep. 4, 271-286.

Santos,A., Humayun,M.S., de Juan E Jr, Greenburg,R.J., Marsh,M.J., Klock,I.B., and Milam,A.H. (1997). Preservation of the inner retina in retinitis pigmentosa. A morphometric analysis. Arch. Ophthalmol. 115, 511-515.

Santos-Ferreira,T., Postel,K., Stutzki,H., Kurth,T., Zeck,G., and Ader,M. (2014). Daylight vision repair by cell transplantation. Stem Cells.

Schlichtenbrede,F.C., Macneil,A., Bainbridge,J.W., Tschernutter,M., Thrasher,A.J., Smith,A.J., and Ali,R.R. (2003). Intraocular gene delivery of ciliary neurotrophic factor results in significant loss of retinal function in normal mice and in the Prph2Rd2/Rd2 model of retinal degeneration. Gene Ther. 10, 523-527.

Seiler,M.J. and Aramant,R.B. (2012). Cell replacement and visual restoration by retinal sheet transplants. Prog. Retin. Eye Res. 31, 661-687.

Silver,I.A., Deas,J., and Erecinska,M. (1997). Ion homeostasis in brain cells: differences in intracellular ion responses to energy limitation between cultured neurons and glial cells. Neuroscience 78, 589-601.

Silverman,M.S. and Hughes,S.E. (1989a). Photoreceptor transplantation in inherited and environmentally induced retinal degeneration: anatomy, immunohistochemistry and function. Prog. Clin. Biol. Res. 314, 687-704.

Silverman,M.S. and Hughes,S.E. (1989b). Transplantation of photoreceptors to lightdamaged retina. Invest Ophthalmol. Vis. Sci. 30, 1684-1690.

Silverman,M.S. and Hughes,S.E. (1990). Photoreceptor rescue in the RCS rat without pigment epithelium transplantation. Curr. Eye Res. 9, 183-191.

Silverman,M.S., Hughes,S.E., Valentino,T.L., and Liu,Y. (1992). Photoreceptor transplantation: anatomic, electrophysiologic, and behavioral evidence for the functional reconstruction of retinas lacking photoreceptors. Exp. Neurol. 115, 87-94.

Singh,M.S., Charbel,I.P., Butler,R., Martin,C., Lipinski,D.M., Sekaran,S., Barnard,A.R., and MacLaren,R.E. (2013a). Reversal of end-stage retinal degeneration and restoration of visual function by photoreceptor transplantation. Proc. Natl. Acad. Sci. U. S. A 110, 11011106. 
Singh,M.S., Charbel,I.P., Butler,R., Martin,C., Lipinski,D.M., Sekaran,S., Barnard,A.R., and MacLaren,R.E. (2013b). Reversal of end-stage retinal degeneration and restoration of visual function by photoreceptor transplantation. Proc. Natl. Acad. Sci. U. S. A 110, 11011106.

Singhal,S., Bhatia,B., Jayaram,H., Becker,S., Jones,M.F., Cottrill,P.B., Khaw,P.T., Salt,T.E., and Limb,G.A. (2012). Human Muller glia with stem cell characteristics differentiate into retinal ganglion cell (RGC) precursors in vitro and partially restore RGC function in vivo following transplantation. Stem Cells Transl. Med. 1, 188-199.

Smith,A.J., Bainbridge,J.W., and Ali,R.R. (2012). Gene supplementation therapy for recessive forms of inherited retinal dystrophies. Gene Ther. 19, 154-161.

Smith,W.C. and Harland,R.M. (1991). Injected Xwnt-8 RNA acts early in Xenopus embryos to promote formation of a vegetal dorsalizing center. Cell 67, 753-765.

Smith,W.C. and Harland,R.M. (1992). Expression cloning of noggin, a new dorsalizing factor localized to the Spemann organizer in Xenopus embryos. Cell 70, 829-840.

Sohocki,M.M., Daiger,S.P., Bowne,S.J., Rodriquez,J.A., Northrup,H., Heckenlively,J.R., Birch,D.G., Mintz-Hittner,H., Ruiz,R.S., Lewis,R.A. et al. (2001). Prevalence of mutations causing retinitis pigmentosa and other inherited retinopathies. Hum. Mutat. 17, 42-51.

Sridhar,A., Steward,M.M., and Meyer,J.S. (2013). Nonxenogeneic growth and retinal differentiation of human induced pluripotent stem cells. Stem Cells Transl. Med. 2, 255-264.

Srinivas,M., Ng,L., Liu,H., Jia,L., and Forrest,D. (2006a). Activation of the blue opsin gene in cone photoreceptor development by retinoid-related orphan receptor beta. Mol. Endocrinol. 20, 1728-1741.

Srinivas,M., Ng,L., Liu,H., Jia,L., and Forrest,D. (2006b). Activation of the blue opsin gene in cone photoreceptor development by retinoid-related orphan receptor beta. Mol. Endocrinol. 20, 1728-1741.

Stingl,K. and Zrenner,E. (2013). Electronic approaches to restitute vision in patients with neurodegenerative diseases of the retina. Ophthalmic Res. 50, 215-220.

Stone,J., Maslim,J., Valter-Kocsi,K., Mervin,K., Bowers,F., Chu,Y., Barnett,N., Provis,J., Lewis,G., Fisher,S.K. et al. (1999). Mechanisms of photoreceptor death and survival in mammalian retina. Prog. Retin. Eye Res. 18, 689-735.

Straznicky,K. and Gaze,R.M. (1971). The growth of the retina in Xenopus laevis: an autoradiographic study. J. Embryol. Exp. Morphol. 26, 67-79.

Streit,A., Berliner,A.J., Papanayotou,C., Sirulnik,A., and Stern,C.D. (2000). Initiation of neural induction by FGF signalling before gastrulation. Nature 406, 74-78.

Stroeva,O.G. and Mitashov,V.I. (1983). Retinal pigment epithelium: proliferation and differentiation during development and regeneration. Int. Rev. Cytol. 83, 221-293. 
Suzuki,T., Akimoto,M., Imai,H., Ueda,Y., Mandai,M., Yoshimura,N., Swaroop,A., and Takahashi,M. (2007). Chondroitinase ABC treatment enhances synaptogenesis between transplant and host neurons in model of retinal degeneration. Cell Transplant. 16, 493-503.

Swaroop,A., Kim,D., and Forrest,D. (2010). Transcriptional regulation of photoreceptor development and homeostasis in the mammalian retina. Nat. Rev. Neurosci. 11, 563-576.

Tackenberg,M.A., Tucker,B.A., Swift,J.S., Jiang,C., Redenti,S., Greenberg,K.P., Flannery,J.G., Reichenbach,A., and Young,M.J. (2009). Muller cell activation, proliferation and migration following laser injury. Mol. Vis. 15, 1886-1896.

Takahashi,K., Tanabe,K., Ohnuki,M., Narita,M., Ichisaka,T., Tomoda,K., and Yamanaka,S. (2007). Induction of pluripotent stem cells from adult human fibroblasts by defined factors. Cell 131, 861-872.

Takahashi,K. and Yamanaka,S. (2006). Induction of pluripotent stem cells from mouse embryonic and adult fibroblast cultures by defined factors. Cell 126, 663-676.

Takeda,M., Takamiya,A., Jiao,J.W., Cho,K.S., Trevino,S.G., Matsuda,T., and Chen,D.F. (2008). alpha-Aminoadipate induces progenitor cell properties of Muller glia in adult mice. Invest Ophthalmol. Vis. Sci. 49, 1142-1150.

Tanaka,E.M. and Reddien,P.W. (2011). The cellular basis for animal regeneration. Dev. Cell 21, 172-185.

Taylor,H.R. (2005). Diabetic retinopathy. Clin. Experiment. Ophthalmol. 33, 3-4.

Terada,N., Hamazaki,T., Oka,M., Hoki,M., Mastalerz,D.M., Nakano,Y., Meyer,E.M., Morel,L., Petersen,B.E., and Scott,E.W. (2002). Bone marrow cells adopt the phenotype of other cells by spontaneous cell fusion. Nature 416, 542-545.

Theocharis,A.D., Skandalis,S.S., Tzanakakis,G.N., and Karamanos,N.K. (2010). Proteoglycans in health and disease: novel roles for proteoglycans in malignancy and their pharmacological targeting. FEBS J. 277, 3904-3923.

Thomson,J.A., Itskovitz-Eldor,J., Shapiro,S.S., Waknitz,M.A., Swiergiel,J.J., Marshall,V.S., and Jones,J.M. (1998). Embryonic stem cell lines derived from human blastocysts. Science 282, 1145-1147.

Thummel,R., Enright,J.M., Kassen,S.C., Montgomery,J.E., Bailey,T.J., and Hyde,D.R. (2010). Pax6a and Pax6b are required at different points in neuronal progenitor cell proliferation during zebrafish photoreceptor regeneration. Exp. Eye Res. 90, 572-582.

Tropepe,V., Coles,B.L., Chiasson,B.J., Horsford,D.J., Elia,A.J., McInnes,R.R., and Van Der Kooy,D. (2000). Retinal stem cells in the adult mammalian eye. Science 287, 20322036.

Tsonis,P.A. and Del Rio-Tsonis,K. (2004). Lens and retina regeneration: transdifferentiation, stem cells and clinical applications. Exp. Eye Res. 78, 161-172. 
Tucker,B.A., Anfinson,K.R., Mullins,R.F., Stone,E.M., and Young,M.J. (2013). Use of a synthetic xeno-free culture substrate for induced pluripotent stem cell induction and retinal differentiation. Stem Cells Transl. Med. 2, 16-24.

Tucker,B.A., Park,I.H., Qi,S.D., Klassen,H.J., Jiang,C., Yao,J., Redenti,S., Daley,G.Q., and Young,M.J. (2011). Transplantation of adult mouse iPS cell-derived photoreceptor precursors restores retinal structure and function in degenerative mice. PLoS. One. 6, e18992.

Turner,D.L. and Cepko,C.L. (1987). A common progenitor for neurons and glia persists in rat retina late in development. Nature 328, 131-136.

Turner,D.L., Snyder,E.Y., and Cepko,C.L. (1990). Lineage-independent determination of cell type in the embryonic mouse retina. Neuron 4, 833-845.

Ueda,Y., Mizuno,N., and Araki,M. (2012). Transgenic Xenopus laevis with the ef1-alpha promoter as an experimental tool for amphibian retinal regeneration study. Genesis. 50, 642650.

Van Hoffelen,S.J., Young,M.J., Shatos,M.A., and Sakaguchi,D.S. (2003). Incorporation of murine brain progenitor cells into the developing mammalian retina. Invest Ophthalmol. Vis. Sci. 44, 426-434.

Varga,Z.M., Wegner,J., and Westerfield,M. (1999). Anterior movement of ventral diencephalic precursors separates the primordial eye field in the neural plate and requires cyclops. Development 126, 5533-5546.

Vugler,A.A. (2010). Progress toward the maintenance and repair of degenerating retinal circuitry. Retina 30, 983-1001.

Wachs,F.P., Couillard-Despres,S., Engelhardt,M., Wilhelm,D., Ploetz,S., Vroemen,M., Kaesbauer,J., Uyanik,G., Klucken,J., Karl,C. et al. (2003). High efficacy of clonal growth and expansion of adult neural stem cells. Lab Invest 83, 949-962.

Wall,D.S., Mears,A.J., McNeill,B., Mazerolle,C., Thurig,S., Wang,Y., Kageyama,R., and Wallace,V.A. (2009). Progenitor cell proliferation in the retina is dependent on Notchindependent Sonic hedgehog/Hes1 activity. J. Cell Biol. 184, 101-112.

Wan,J., Ramachandran,R., and Goldman,D. (2012). HB-EGF is necessary and sufficient for Muller glia dedifferentiation and retina regeneration. Dev. Cell 22, 334-347.

Wan,J., Zheng,H., Chen,Z.L., Xiao,H.L., Shen,Z.J., and Zhou,G.M. (2008). Preferential regeneration of photoreceptor from Muller glia after retinal degeneration in adult rat. Vision Res. 48, 223-234.

Wan,J., Zheng,H., Xiao,H.L., She,Z.J., and Zhou,G.M. (2007). Sonic hedgehog promotes stem-cell potential of Muller glia in the mammalian retina. Biochem. Biophys. Res. Commun. 363, 347-354. 
Wang,H., Lau,B.W., Yau,S.Y., Li,S.Y., Leung,N., Wang,N.L., Tang,S.W., Lee,T.M., and So,K.F. (2010). Roles of paroxetine and corticosterone on adult mammalian ciliary body cell proliferation. Chin Med. J. (Engl. ) 123, 1305-1310.

Warfvinge,K., Kiilgaard,J.F., Klassen,H., Zamiri,P., Scherfig,E., Streilein,W., Prause,J.U., and Young,M.J. (2006). Retinal progenitor cell xenografts to the pig retina: immunological reactions. Cell Transplant. 15, 603-612.

Warre-Cornish,K., Barber,A.C., Sowden,J.C., Ali,R.R., and Pearson,R.A. (2014). Migration, integration and maturation of photoreceptor precursors following transplantation in the mouse retina. Stem Cells Dev. 23, 941-954.

Wassarman,K.M., Lewandoski,M., Campbell,K., Joyner,A.L., Rubenstein,J.L., Martinez,S., and Martin,G.R. (1997). Specification of the anterior hindbrain and establishment of a normal mid/hindbrain organizer is dependent on Gbx2 gene function. Development 124, 2923-2934.

Watanabe,K., Kamiya,D., Nishiyama,A., Katayama,T., Nozaki,S., Kawasaki,H., Watanabe,Y., Mizuseki,K., and Sasai,Y. (2005). Directed differentiation of telencephalic precursors from embryonic stem cells. Nat. Neurosci. 8, 288-296.

Wawersik,S. and Maas,R.L. (2000). Vertebrate eye development as modeled in Drosophila. Hum. Mol. Genet. 9, 917-925.

Weimann,J.M., Johansson,C.B., Trejo,A., and Blau,H.M. (2003). Stable reprogrammed heterokaryons form spontaneously in Purkinje neurons after bone marrow transplant. Nat. Cell Biol. 5, 959-966.

Wen,R., Tao,W., Li,Y., and Sieving,P.A. (2012). CNTF and retina. Prog. Retin. Eye Res. 31, 136-151.

Wenkel,H. and Streilein,J.W. (1998). Analysis of immune deviation elicited by antigens injected into the subretinal space. Invest Ophthalmol. Vis. Sci. 39, 1823-1834.

Wernig,M., Meissner,A., Foreman,R., Brambrink,T., Ku,M., Hochedlinger,K., Bernstein,B.E., and Jaenisch,R. (2007). In vitro reprogramming of fibroblasts into a pluripotent ES-cell-like state. Nature 448, 318-324.

West,E.L., Gonzalez-Cordero,A., Hippert,C., Osakada,F., Martinez-Barbera,J.P., Pearson,R.A., Sowden,J.C., Takahashi,M., and Ali,R.R. (2012). Defining the integration capacity of embryonic stem cell-derived photoreceptor precursors. Stem Cells 30, 14241435.

West,E.L., Pearson,R.A., Barker,S.E., Luhmann,U.F., MacLaren,R.E., Barber,A.C., Duran,Y., Smith,A.J., Sowden,J.C., and Ali,R.R. (2010). Long-term survival of photoreceptors transplanted into the adult murine neural retina requires immune modulation. Stem Cells 28, 1997-2007. 
West,E.L., Pearson,R.A., Tschernutter,M., Sowden,J.C., MacLaren,R.E., and Ali,R.R. (2008). Pharmacological disruption of the outer limiting membrane leads to increased retinal integration of transplanted photoreceptor precursors. Exp. Eye Res. 86, 601-611.

Wichterle,H., Lieberam,I., Porter,J.A., and Jessell,T.M. (2002). Directed differentiation of embryonic stem cells into motor neurons. Cell 110, 385-397.

WohI,S.G., Schmeer,C.W., Kretz,A., Witte,O.W., and Isenmann,S. (2009). Optic nerve lesion increases cell proliferation and nestin expression in the adult mouse eye in vivo. Exp. Neurol. 219, 175-186.

Wojciechowski,A.B., Englund,U., Lundberg,C., Wictorin,K., and Warfvinge,K. (2002). Subretinal transplantation of brain-derived precursor cells to young RCS rats promotes photoreceptor cell survival. Exp. Eye Res. 75, 23-37.

Wolda,S.L., Moody,C.J., and Moon,R.T. (1993). Overlapping expression of Xwnt-3A and Xwnt-1 in neural tissue of Xenopus laevis embryos. Dev. Biol. 155, 46-57.

Wood,H.B. and Episkopou,V. (1999). Comparative expression of the mouse Sox1, Sox2 and Sox3 genes from pre-gastrulation to early somite stages. Mech. Dev. 86, 197-201.

Xu,H., Sta Iglesia,D.D., Kielczewski,J.L., Valenta,D.F., Pease,M.E., Zack,D.J., and Quigley,H.A. (2007a). Characteristics of progenitor cells derived from adult ciliary body in mouse, rat, and human eyes. Invest Ophthalmol. Vis. Sci. 48, 1674-1682.

Xu,S., Sunderland,M.E., Coles,B.L., Kam,A., Holowacz,T., Ashery-Padan,R., Marquardt,T., Mclnnes,R.R., and Van Der Kooy,D. (2007b). The proliferation and expansion of retinal stem cells require functional Pax6. Dev. Biol. 304, 713-721.

Xue,L.P., Lu,J., Cao,Q., Kaur,C., and Ling,E.A. (2006). Nestin expression in Muller glial cells in postnatal rat retina and its upregulation following optic nerve transection. Neuroscience 143, 117-127.

Yamaguchi,M., Saito,H., Suzuki,M., and Mori,K. (2000). Visualization of neurogenesis in the central nervous system using nestin promoter-GFP transgenic mice. Neuroreport 11, 1991-1996.

Yanagi,Y., Inoue,Y., Kawase,Y., Uchida,S., Tamaki,Y., Araie,M., and Okochi,H. (2006). Properties of growth and molecular profiles of rat progenitor cells from ciliary epithelium. Exp. Eye Res. 82, 471-478.

Yang,P., Seiler,M.J., Aramant,R.B., and Whittemore,S.R. (2002). In vitro isolation and expansion of human retinal progenitor cells. Exp. Neurol. 177, 326-331.

Yaron,O., Farhy,C., Marquardt,T., Applebury,M., and Ashery-Padan,R. (2006). Notch1 functions to suppress cone-photoreceptor fate specification in the developing mouse retina. Development 133, 1367-1378.

Ying,Q.L., Nichols,J., Evans,E.P., and Smith,A.G. (2002). Changing potency by spontaneous fusion. Nature 416, 545-548. 
Ying,Q.L., Stavridis,M., Griffiths,D., Li,M., and Smith,A. (2003). Conversion of embryonic stem cells into neuroectodermal precursors in adherent monoculture. Nat. Biotechnol. 21, 183-186.

Yoshikawa,T., Mizuno,A., Yasumuro,H., Inami,W., Vergara,M.N., Del Rio-Tsonis,K., and Chiba,C. (2012). MEK-ERK and heparin-susceptible signaling pathways are involved in cellcycle entry of the wound edge retinal pigment epithelium cells in the adult newt. Pigment Cell Melanoma Res. 25, 66-82.

Young,M.J., Ray,J., Whiteley,S.J., Klassen,H., and Gage,F.H. (2000). Neuronal differentiation and morphological integration of hippocampal progenitor cells transplanted to the retina of immature and mature dystrophic rats. Mol. Cell Neurosci. 16, 197-205.

Young,R.W. (1985). Cell differentiation in the retina of the mouse. Anat. Rec. 212, 199-205.

Young,T.L. and Cepko,C.L. (2004). A role for ligand-gated ion channels in rod photoreceptor development. Neuron 41, 867-879.

Yurco,P. and Cameron,D.A. (2005). Responses of Muller glia to retinal injury in adult zebrafish. Vision Res. 45, 991-1002.

Yurco,P. and Cameron,D.A. (2007). Cellular correlates of proneural and Notch-delta gene expression in the regenerating zebrafish retina. Vis. Neurosci. 24, 437-443.

Zhang,S.C., Goetz,B.D., and Duncan,I.D. (2003). Suppression of activated microglia promotes survival and function of transplanted oligodendroglial progenitors. Glia 41, 191198.

Zhao,J.J., Ouyang,H., Luo,J., Patel,S., Xue,Y., Quach,J., Sfeir,N., Zhang,M., Fu,X., Ding,S. et al. (2014). Induction of retinal progenitors and neurons from mammalian Muller glia under defined conditions. J. Biol. Chem. 289, 11945-11951.

Zhao,S., Hung,F.C., Colvin,J.S., White,A., Dai,W., Lovicu,F.J., Ornitz,D.M., and Overbeek,P.A. (2001). Patterning the optic neuroepithelium by FGF signaling and Ras activation. Development 128, 5051-5060.

Zhao,X., Das,A.V., Soto-Leon,F., and Ahmad,I. (2005). Growth factor-responsive progenitors in the postnatal mammalian retina. Dev. Dyn. 232, 349-358.

Zhao,X., Liu,J., and Ahmad,I. (2002). Differentiation of embryonic stem cells into retinal neurons. Biochem. Biophys. Res. Commun. 297, 177-184.

Zhong,X., Gutierrez,C., Xue,T., Hampton,C., Vergara,M.N., Cao,L.H., Peters,A., Park,T.S., Zambidis,E.T., Meyer,J.S. et al. (2014). Generation of three-dimensional retinal tissue with functional photoreceptors from human iPSCs. Nat. Commun. 5, 4047.

Zhou,H., Wu,S., Joo,J.Y., Zhu,S., Han,D.W., Lin,T., Trauger,S., Bien,G., Yao,S., Zhu,Y. et al. (2009). Generation of induced pluripotent stem cells using recombinant proteins. Cell Stem Cell 4, 381-384. 
Zhou,L., Wang,W., Liu,Y., Fernandez de,C.J., Ezashi,T., Telugu,B.P., Roberts,R.M., Kaplan,H.J., and Dean,D.C. (2011). Differentiation of induced pluripotent stem cells of swine into rod photoreceptors and their integration into the retina. Stem Cells 29, 972-980.

Zuber,M.E., Gestri,G., Viczian,A.S., Barsacchi,G., and Harris,W.A. (2003). Specification of the vertebrate eye by a network of eye field transcription factors. Development 130, 51555167. 


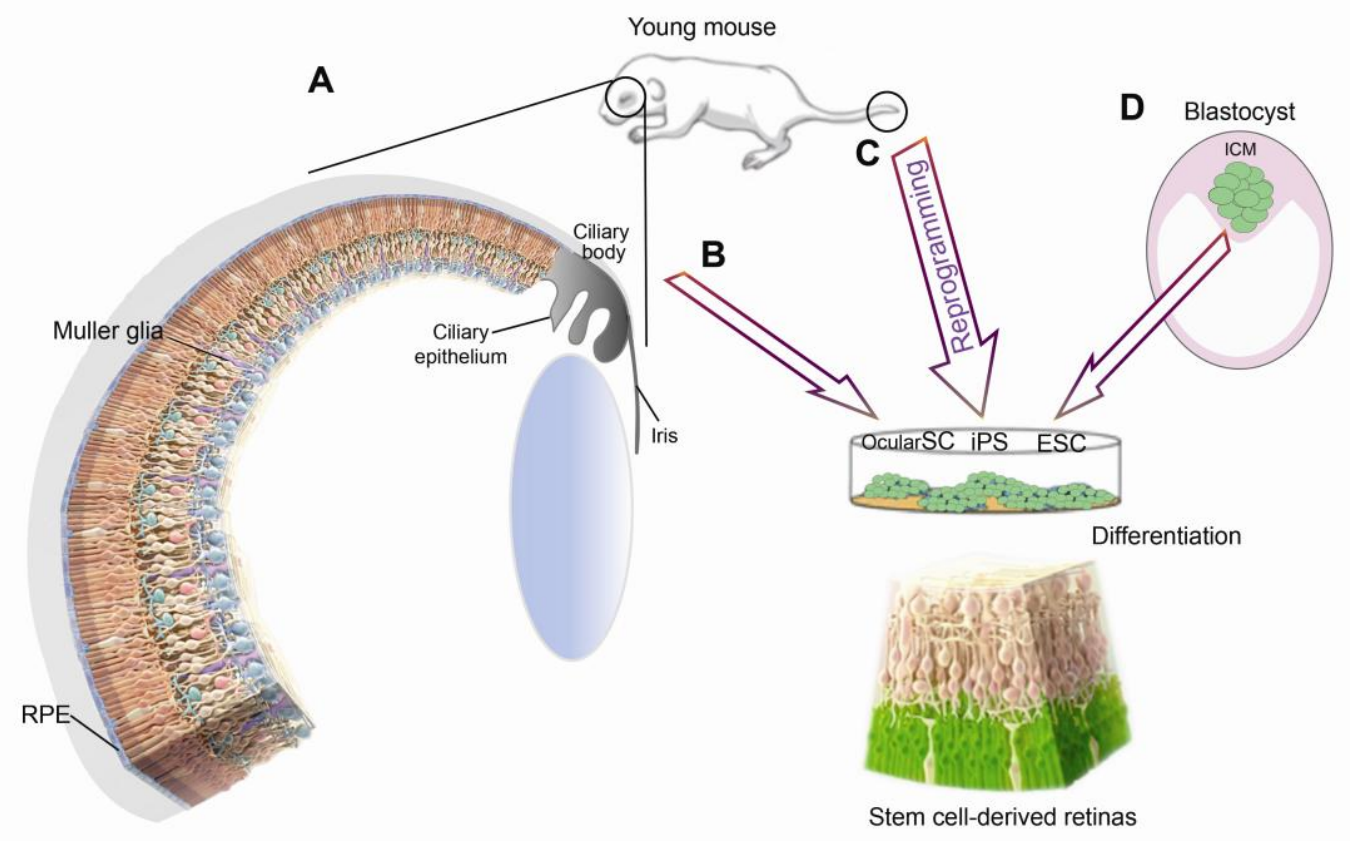

Figure 1. Sources of cells for cell-based therapies.

A. Schematic of a mouse eye demonstrating the main sources of endogenous stem/progenitor cells in the eye, which includes the ciliary epithelium (CE), iris, retinal pigment epithelium (RPE) and Müller glia cells. B. These stem/progenitor cell populations also represent an attractive source for cell transplantation studies as they can be expanded and cultured in vitro towards a more photoreceptor phenotype. In addition to these ocular stem cells populations $\mathbf{C}$. somatic cells, which can be reprogrammed into iPS cells, and $\mathbf{D}$. embryonic stem cells (derived from the inner cell mass of the blastocyst) can be isolated, expanded and kept in undifferentiated conditions in vitro, prior to differentiation into retinal cell types, including photoreceptors, suitable for cell replacement. 


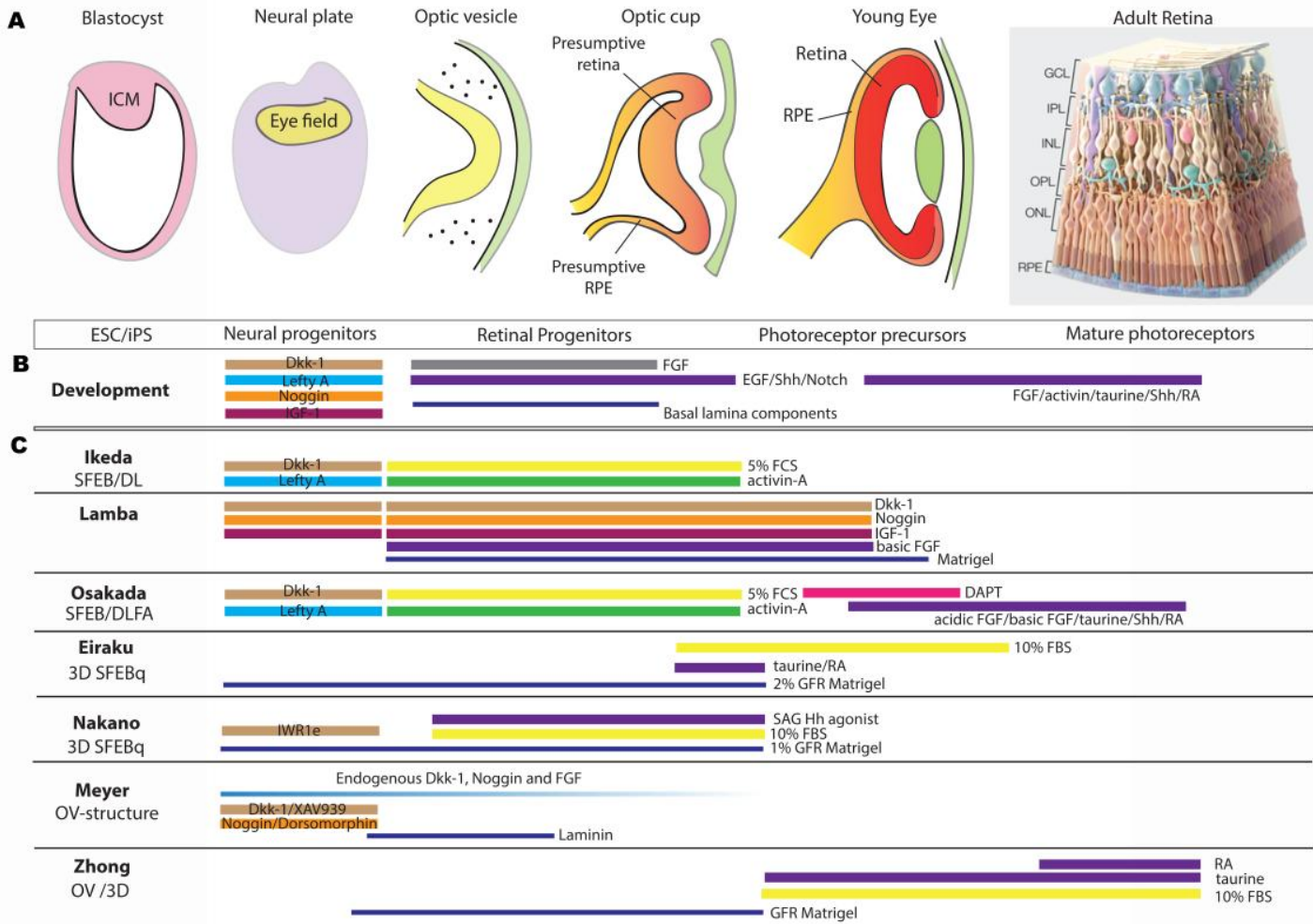

Figure 2. Eye development and differentiation of pluripotent stem cell protocols.

A. Schematic demonstrating the various stages of eye development, from the blastocyst stage through to the mature adult retina. At very early stages, the eye field domain is specified from a region of the neural plate. This region extends laterally to form the optic vesicle, and then invaginates to from the optic cup. Further specification of this region gives rise to the young eye, which contains a two-layered optic cup; at this stage, the developing neural retina (red) is situated in the inner region of this structure, whereas the RPE forms the outer RPE layer (orange). Further differentiation gives rise to the mature retina containing all retinal cell types, distributed in their correct laminar position. B. Summary of the main signaling factors required for normal eye development, from neural progenitors to retinal progenitors and finally photoreceptor precursors. C. Representation of the signaling factors used in some of the key ESC/iPS differentiation protocols published to date. 


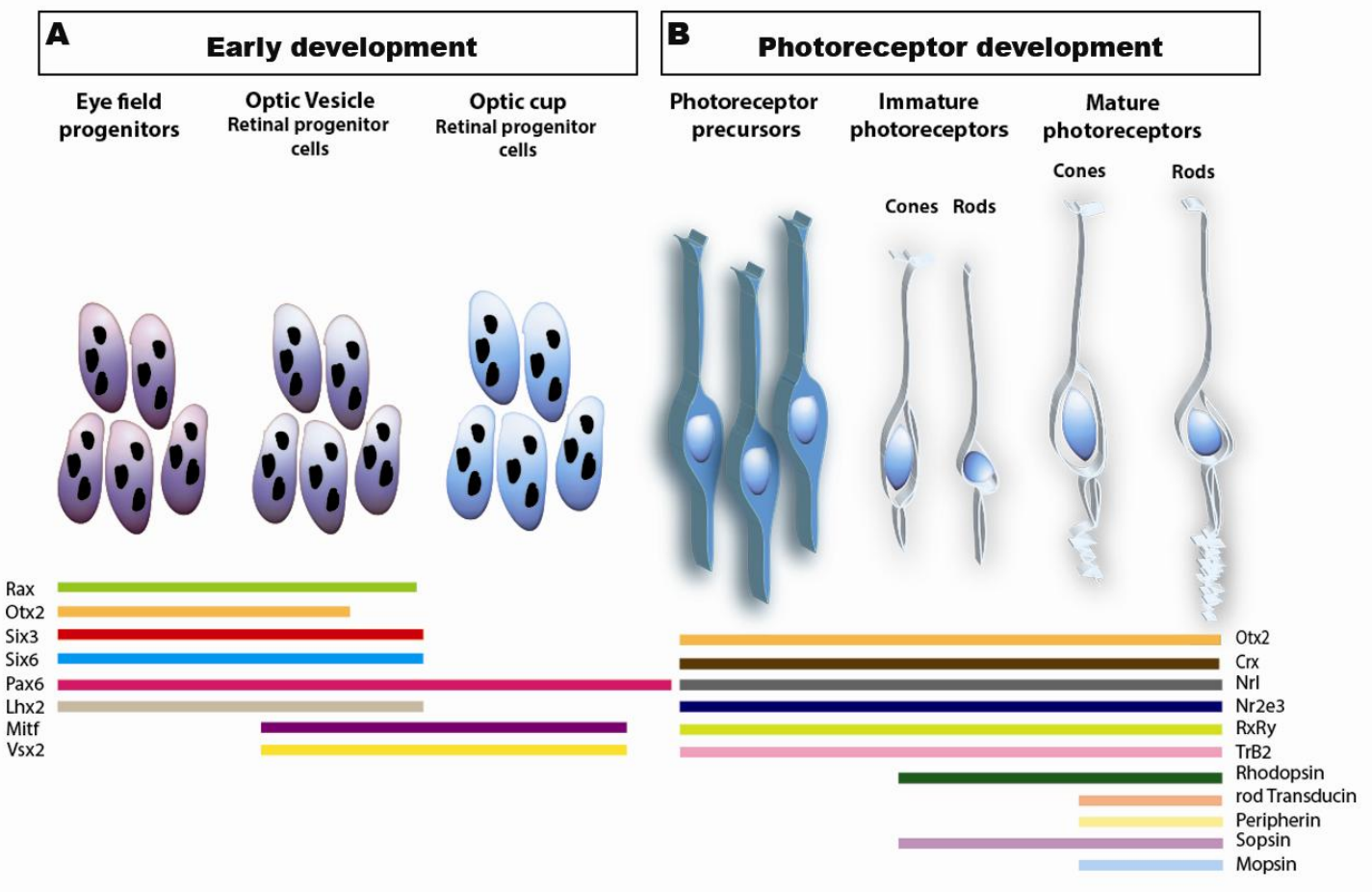

Figure 3. Transcriptional control of photoreceptor development.

A. Schematic representation of the developmental cascade of genes implicated in early eye development, with particular reference to B. photoreceptor cell differentiation 


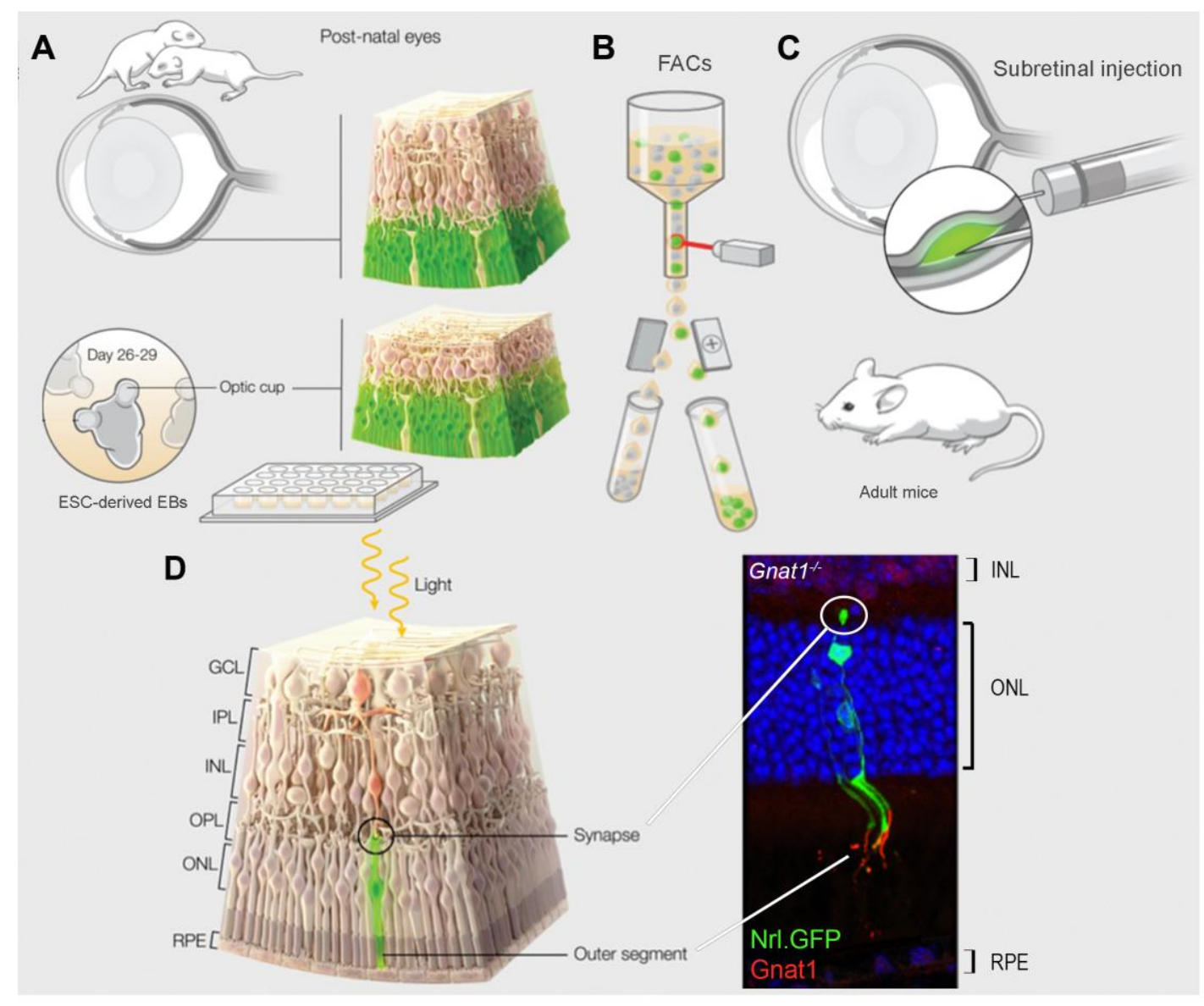

Figure 4. Transplantation of photoreceptors precursors into the adult retina.

A. Two main sources of cells for transplantation include photoreceptor precursors from the postnatal retina and differentiated embryonic stem cells (see Gonzalez-Cordero et al., 2013 and Pearson et al., 2012). In each case, the photoreceptors precursors are labeled, typically with fluorescent reporters. B. The whole retina and/or ESC-derived EBs are dissociated and the photoreceptors are isolate and purified by Fluorescent Activated Cell sorting (FACs). C. These purified GFP-positive population of photoreceptors (200,000 cells per injection) are then subretinally transplanted between the ONL and RPE. D. Schematic and confocal image of integrated GFP-positive mature photoreceptors in the ONL. Six weeks following transplantation into a rod $\alpha$ transducing knockout (Gnat ${ }^{-1}$ ) animal Nrl.GFP photoreceptors form rod $\alpha$-transducin-expressing outer segments and synaptic buttons connecting with the endogenous circuitry. These cells can then rescue visual function when excited by light.

ONL: outer nuclear layer; INL: inner nuclear layer; RPE: retinal pigmented epithelium; OPL: outer plexiform layer; IPL: inner plexiform layer; GCL: ganglion cell layer. 